cond-mat/9711014

\title{
Spin dynamics and transport in gapped one-dimensional Heisenberg antiferromagnets at nonzero temperatures
}

\author{
Kedar Damle and Subir Sachdev \\ Department of Physics, P.O. Box 208120, Yale University, New Haven, CT 06520-8120
}

(October 29, 1997)

\begin{abstract}
We present the theory of nonzero temperature $(T)$ spin dynamics and transport in one-dimensional Heisenberg antiferromagnets with an energy gap $\Delta$. For $T \ll \Delta$, we develop a semiclassical picture of thermally excited particles. Multiple inelastic collisions between the particles are crucial, and are described by a two-particle $\mathcal{S}$-matrix which has a super-universal form at low momenta. This is established by computations on the $O(3) \sigma$-model, and strong and weak coupling expansions (the latter using a Majorana fermion representation) for the two-leg $S=1 / 2$ Heisenberg antiferromagnetic ladder. As an aside, we note that the strong-coupling calculation reveals a $S=1$, two particle bound state which leads to the presence of a second peak in the $T=0$ inelastic neutron scattering (INS) cross-section for a range of values of momentum transfer. We obtain exact, or numerically exact, universal expressions for the thermal broadening of the quasi-particle peak in the INS cross-section, for the magnetization transport, and for the field dependence of the NMR relaxation rate $1 / T_{1}$ of the effective semiclassical model: these are expected to be asymptotically exact for the quantum antiferromagnets. The results for $1 / T_{1}$ are compared with the experimental findings of Takigawa et.al. and the agreement is quite good. In the regime $\Delta<T<$ (a typical microscopic exchange) we argue that a complementary description in terms of semiclassical waves applies, and give some exact results for the thermodynamics and dynamics.
\end{abstract}

Typeset using REVTEX 


\section{INTRODUCTION}

For more than a decade now, much effort has been devoted to understanding the properties of a variety of insulating one-dimensional Heisenberg antiferromagnets. By now, the basic facts about these systems are very well established: Heisenberg antiferromagnetic (HAF) chains with integer spins at each site exhibit a gap in their excitation spectrum while those with half- integer spins are gapless [1,2]. Among the spin-1/2 ladder compounds, those with an even number of legs exhibit a gap just like the integer spin chains [3,4, 5] while ladders with an odd number of legs are gapless analogous to the half-integer chains [5].

Theoretically, the universal low-energy properties of the gapped systems are well described by the one dimensional quantum $O(3)$ non-linear $\sigma$-model (NL $\sigma \mathrm{M}$ ) without any topological term [6, [1,8]. A lot is known exactly about this field theory [9, 10,11] and this is directly useful in understanding the gapped systems. The spectrum of the $\sigma$-model consists of a triplet of massive spin-1 particles as the lowest energy excitations followed by multiparticle continua with no bound states. Many zero temperature $(T)$ properties of the gapped systems, including low frequency dynamic correlations, can be explained using the exact information available on the $\sigma$-model [12]. On the other hand, until very recently exact results for $T>0$ were restricted to static, thermodynamic properties [13] while many experimental observables (such as the inelastic neutron scattering (INS) crosssection and NMR relaxation rates) directly probe dynamical correlations at non-zero temperature.

(The universal low-energy properties of the gapless systems have been treated via a mapping to a certain critical field theory [14]. In contrast to the NL $\sigma \mathrm{M}$, powerful techniques that exploit the conformal invariance of the theory can be used to determine exactly for $T>0$ some dynamical correlators that are directly probed by NMR experiments [15, 16, 17. Similar methods have been used to obtain results for $T>0$ on static properties as well 18 . Transport properties have also been studied recently [19,20,21], with results that are quite different from those we shall obtain here for gapful systems.)

This paper shall deal exclusively with the $T>0$ dynamical properties of gapped Heisenberg spin chains. A portion of our results have appeared earlier in a short report [22], where we presented them in the context of the continuum NL $\sigma \mathrm{M}$, but did not fully discuss their range of applicability. Here we shall take a more general point of view of working directly with lattice Heisenberg antiferromagnets. The main, and essentially only, requirement on the spin chain being studied is that it have an energy gap and that its low-lying excitations consist of a triplet of spin-1 particles with the dispersion

$$
\varepsilon(k)=\Delta+\frac{c^{2} k^{2}}{2 \Delta}+\mathcal{O}\left(k^{4}\right) \ldots
$$

Here $k$ is being measured from an antiferromagnetic wavevector $Q=\pi / a(k=q-Q$, and $a$ is the lattice spacing), and we have introduced a velocity $c$ to parametrize the mass of the particles as $\Delta / c^{2}$. This is in keeping with the 'relativistic' spectrum of the $O(3) \sigma$-model $\varepsilon(k)=\left(\Delta^{2}+c^{2} k^{2}\right)^{1 / 2}$, although most of our results will not rely on this relativistic form. Gapped spin chains with a spontaneously broken translational symmetry (spin-Peierls order) can have spin-1/2 particle excitations: we shall not deal with this case explicitly, although we believe most of our results can also be extended to these systems. 
The energy gap $\Delta$ is an important energy scale which shall play a central role in our analysis. The majority of our results will be in the regime $T \ll \Delta$ (we shall use units with $\hbar=k_{B}=1$ throughout) which we now discuss.

In this regime, there is a dilute gas of excited particles present, and their motion and collisions dominate the dynamical properties we study. In particular their spacing $\sim c e^{\Delta / T} /(\Delta T)^{1 / 2}$ is much larger than their thermal de-Broglie wavelength $\sim c /(\Delta T)^{1 / 2}$. As argued in Ref [23], these particles can be treated classically except when two of them collide. Such two-particle collisions need to be treated quantum-mechanically and are described by an $\mathcal{S}$-matrix, which is, in general, a complicated function of the particle momenta and spin orientations. Conservation of total momentum and energy implies that momenta before and after collisions have to be the same, and $O(3)$ invariance and unitarity impose further constraints, but a fairly complex structure is still permitted-we will see some explicit examples in this paper. However, the r.m.s. thermal velocity of a particle $v_{T}=(T / \Delta)^{1 / 2} c \rightarrow 0$ as $T / \Delta \rightarrow 0$ and thus we need the $\mathcal{S}$-matrix only in the limit of vanishing incoming (and outgoing) momenta. One of the central ingredients in our computations will be our claim that in this limit, all of the complexity disappears, and the $\mathcal{S}$-matrix has a super-universal form; for the scattering event shown in Fig 1 we have (here $\mu_{i}=x, y, z$ are the three possible values of the $O(3)$ spin label)

$$
\mathcal{S}_{\mu_{1}^{\prime}, \mu_{2}^{\prime}}^{\mu_{1} \mu_{2}}\left(k_{1}, k_{2} ; k_{1}^{\prime}, k_{2}^{\prime}\right)=(-1) \delta_{\mu_{1} \mu_{2}^{\prime}} \delta_{\mu_{2} \mu_{1}^{\prime}}(2 \pi)^{2} \delta\left(k_{1}-k_{1}^{\prime}\right) \delta\left(k_{2}-k_{2}^{\prime}\right) ;
$$

Notice especially the opposite pairing of momentum and spin labels: crudely speaking, the momenta go "through" the collision, while the spins "bounce off" - this dichotomy will be crucial to our considerations. We dub this limiting value of the $\mathcal{S}$-matrix 'super-universal' as it requires only that the lowest lying excitations above the gap satisfy (1.1) at low momenta. The value, however, does not depend on parameters such as $c$ and $\Delta$. Moreover, we expect this limiting result to hold even at the lattice level for generic microscopic models of onedimensional antiferromagnets with massive spin one excitations; we shall see one explicit example that bears out this expectation later in the paper.

With this simple form of the $\mathcal{S}$-matrix in hand, we will use the semiclassical techniques of Ref [23] to analyze dynamical properties of spin fluctuations near $q=0$ and $q=Q$ in terms of the motion of the dilute gas of quasiparticles.

We will begin by discussing the properties of the $\mathcal{S}$-matrix for two-particle scattering in the limit of low momenta in Section [1]. In Section IIA we consider the $\mathcal{S}$ matrix for the $O(3)$ non-linear sigma model. This has been computed for all momenta by Zamolodchikov and Zamolodchikov, and we shall show that the zero momentum limit does indeed satisfy (11.2).

However, the $\sigma$-model is a continuum theory; it would be much more satisfactory to be able to directly see that (1.2) holds for some specific microscopic model, and explicitly verify that lattice effects do not affect the simple structure of this limit. One such model is the $S=1 / 2$, two-leg Heisenberg antiferromagnetic ladder with inter-chain exchange $J_{\|}$ and intra-chain exchange $J_{\perp}$. The properties of the ladder can be analysed using a 'strongcoupling' expansion [24] in powers of $J_{\|} / J_{\perp}$ for the microscopic lattice Hamiltonian of the system. In Section [1B we shall explicitly verify (1.2) for vanishing velocities in this lattice model within this strong-coupling expansion. Parenthetically, we note that our strongcoupling analysis also allows us to make predictions about interesting features in the $T=0$ 
dynamic structure factor $S(q, \omega)$ which are specific to the system considered. In particular, we find that, to second order in $J_{\|} / J_{\perp}$, a two-particle $S=1$ bound state gives rise to a second peak (in addition to the usual peak coming from the stable single particle excitations of the system) in $S(q, \omega)$ for a range of values of $q$ around $Q$. This should be of relevence to inelastic neutron scattering (INS) experiments on the ladder compounds and it is hoped that they experimentally verify the existence of this effect.

In Section $\mathbb{I C}$ we study the complementary 'weak-coupling' expansion in powers of $J_{\perp} / J_{\|}$for the two-leg ladder. As was shown in Ref [25], this expansion leads to a description of the low-energy, long-distance properties of the ladder in terms of an effective field theory of a triplet of massive Majorana fermions. The Hamiltonian for the Majorana fermions also has a four-fermion coupling which has generally been ignored in previous treatments. In the absence of this scattering, the Majorana fermions are free, and the resulting $\mathcal{S}$-matrix does not obey (1.2). In this paper, we consider the effect of the four-fermion coupling in perturbation theory. We show that this expansion suffers from severe infra-red problems which have to be resolved by an infinite-order resummation. The structure of the divergences is very similar to those also present in the large- $N$ expansion of the $\sigma$-model above, and we find that the resulting resummed $\mathcal{S}$-matrix of the Majorana fermions does indeed obey the analogue of (1.2). So neglecting the four-fermion coupling [26], (or even treating it in an unresummed manner at finite order in perturbation theory) is a very bad approximation at low momenta, and we expect that corresponding divergences in the perturbative evaluation of the spin-spin correlation function invalidate the dynamical results of Ref [26] at low $T$.

In Section III we shall turn to a discussion of the dynamical properties in the regime $0<T \ll \Delta$. Our results apply universally to all gapped one-dimensional antiferromagnetic systems with spin one quasiparticles; indeed they rely only on the dispersion (1.1) and the $\mathcal{S}$-matrix in (1.2). All our results will be expressed solely in terms of the parameters $c$ and $\Delta$, the temperature, $T$ and the external field $H$.

In Section $\Pi 1 \mathrm{~A}$ we study the dynamics of the staggered component (with wavevector $q$ close to $Q$ ) of the fluctuations in the spin density. More precisely, we study the dynamical structure factor $S(q, \omega)$ for $q$ close to $Q$. Apart from some overall factors, this directly gives the INS crosssection at the corresponding values of momentum and energy transfer. At $T=0$, the dynamical structure factor has a sharply defined $\delta$-function peak at $\omega=\varepsilon(q-Q)$ for $q$ near $Q$. This peak can be thought of as arising from the ballistic propogation of the stable quasiparticle of the system. At non-zero temperatures, the peak broadens as the quasiparticle suffers collisions with other thermally excited particles. The main objective of Section IIIA is to describe the precise lineshape of the quasiparticle peak in the dynamic structure factor for $T>0$.

In the $\sigma$-model approach, the staggered components of the spin density are represented by the antiferromagnetic order parameter field $\vec{n}$. We will use the semiclassical method of Ref- 223 to calculate the space and time dependent 2-point correlation function of the $\vec{n}$ field for $T>0$. This allows us to calculate the thermal broadening of the single particle peak in the dynamical structure factor $S(q, \omega)$ for wavevectors $q$ near $Q$. In particular, we find that the dynamic structure factor in the immediate vicinity of the quasiparticle peak at $(q=Q, \omega=\Delta)$ may be written in a reduced scaling form as 


$$
S(q, \omega)=\frac{\mathcal{A} c L_{t}}{\pi^{2} \Delta} \Phi\left(\frac{\omega-\varepsilon(k)}{L_{t}^{-1}}\right),
$$

where $k=q-Q, \mathcal{A}$ is the (non-universal) quasiparticle amplitude of the spin one excitations of the system, $L_{t}=\sqrt{\pi} e^{\Delta / T} / 3 T$ is the typical time spent by a thermally excited quasiparticle between collisions with other particles, and $\Phi$ is a completely universal function that we determine numerically in this paper. Notice that temperature enters this scaling form only through $L_{t}$. We claim, though this is not rigorously established, that these results for the broadening are asymptotically exact for $T \ll \Delta$ : all corrections to the line-width are expected to be suppressed by positive powers of $T / \Delta$. Some evidence for the exactness of our results emerges from consideration of simpler systems where exact results for the linebroadening are available from the quantum inverse scattering method [27]; as we shall see in Section IIIA, our semiclassical results are in perfect agreement [23] with these. It is hoped that experimental studies of the temperature dependence of the INS cross-section in this regime will confirm these results, particularly the simple scaling form (1.3).

In Section IIIB we turn to the correlations of the conserved magnetization density, or dynamic fluctuations near $q=0$, for $T \ll \Delta$. Unlike the staggered case, the overall magnitude of the magnetization density fluctuations is universal and given by $T \chi_{u}$, where $\chi_{u}$ is the uniform susceptibility of the system (the non-universal overall scale of the staggered component is reflected for instance by the presence of the overall constant $\mathcal{A}$ in (1.3)). In this temperature regime, we have the well-known result for $\chi_{u}$ [13]:

$$
\chi_{u}=\frac{1}{c}\left(\frac{2 \Delta}{\pi T}\right)^{1 / 2} e^{-\Delta / T}
$$

We shall study the dynamics of the magnetization density in Section [IIB [22]. We shall show that the long-time correlations of an effective semiclassical model are characterized by spin diffusion, and obtain the following result for its low $T$ spin-diffusion constant $D_{s}$ :

$$
D_{s}=\frac{c^{2}}{3 \Delta} e^{\Delta / T}
$$

Using the Einstein relation for the spin conductivity $\sigma_{s}=D_{s} \chi_{u}$, we obtain from (1.4) and (1.5)

$$
\sigma_{s}=\frac{c}{3}\left(\frac{2}{\pi \Delta T}\right)^{1 / 2}
$$

Notice that the exponentially large factor $e^{\Delta / T}$ has dropped out, and $\sigma_{s}$ diverges with an inverse square-root power in $T$ as $T \rightarrow 0$. The semiclassical model is possesses an infinite number of local conservation laws: in Appendix A, we discuss how the existence of spin diffusion can be compatible with these local conservation laws. However, these results do not rigorously allow us to conclude that the ultimate long-time correlations of the underlying gapped quantum spin chain are diffusive. This has to do with a subtle question of order of limits: we computed the $\mathcal{S}$ matrix (1.2) in the limit $T / \Delta \rightarrow 0$, and then used it to evaluate the long-time limit of correlations of the magnetization, whereas in reality the limits should be taken in the opposite order. What we can claim is that our results will 
apply for all times upto a time scale which is larger than the collision time $L_{t}$ by a factor which diverges with a positive power of $\Delta / T$ as $T \rightarrow 0$; there is a substantial time window in this regime where we have established that the spin correlations are diffusive. For the generic gapped quantum spin chain, we can reasonably expect that the ultimate long-time correlations are indeed diffusive, and the only consequences of the omitted terms in the $\mathcal{S}$ matrix are subdominant corrections to the value of $D_{s}$ in (1.5) which are suppressed by powers of $T / \Delta$. For the continuum $\mathrm{NL} \sigma \mathrm{M}$ with a relativistically invariant regularization (this is unphysical for any experimental application), the issue is a little more subtle: this model does possess additional non-local conserved quantities [28], but we consider it unlikely that these will modify the long time limit [29]. On the experimental side, however, diffusive behaviour of the magnetization density has already been convincingly demonstrated in the $S=1$ one-dimensional antiferromagnet $\mathrm{AgVP}_{2} \mathrm{~S}_{6}$ by the NMR experiments of Takigawa et.al. [30].

As has been argued earlier [31], the dynamic fluctuations near $q=0$ provide the dominant contribution to the NMR relaxation rate $1 / T_{1}$ for $T \ll \Delta$. Thus, knowing the space and time dependent two-point correlation function of the conserved magnetization density, we are able to compute the field and temperature dependent $1 / T_{1}$ in this regime. We shall see that the overall scale of $1 / T_{1}$ is set by the ratio $T \chi_{u} / \sqrt{D_{S}}$. As was pointed out to us by $\mathrm{M}$. Takigawa [22], this immediately leads to an activation gap for $1 / T_{1}$ given as

$$
\Delta_{1 / T_{1}}=\frac{3}{2} \Delta .
$$

This difference between the activation gaps for $\chi_{u}$ and $1 / T_{1}$ appears to clear-up puzzling discrepencies in the experimental literature [30.32.50 for the value of the energy gap in these systems obtained from Knight-shift susceptibilty measurements on the one hand, and $1 / T_{1}$ NMR relaxation rates on the other; a systematic tabulation of the activation gaps for a large number of gapped spin chains [33] does indeed show a trend consistent with (1.7). The crucial factor of $3 / 2$ clearly arises from the exponential divergence in $D_{s}$. This diffusive behaviour we find arises entirely from intrinsic inelastic scattering between the quasi-particles. In real systems there will also be contributions from elastic scattering off inhomogenities which will eventually saturate the divergence of $D_{s}$ as $T \rightarrow 0$. However, because of the strong spin scattering implied by (1.2) the effects of inelastic scattering is particularly strong in $d=1$, and can easily dominate inhomogenities in clean samples.

We will give a detailed account of the calculations leading up to our expression for $1 / T_{1}$ (some details on the method used are relegated to Appendix C) and then go on to compare the theoretical predictions for the field dependence of $1 / T_{1}$ with the extensive experimental data of Takigawa et.al. 30]. We will see that our results (without any adjustable parameters, except for a field independent background rate) agree with the data extremely well for a range of intermediate temperatures. At the lowest temperatures for which data is available, the quality of the fit deteriorates significantly and the $1 / \sqrt{H}$ divergence predicted at small fields seems to get cutoff, presumably by some spin-dissipation mechanism present in the real system. At the present time, we are unable to incorporate this dissipation in any serious way in our approach. However, following Ref [30], we can phenomenologically introduce some spin-dissipation in our results for the long-time limit of the autocorrelation function and obtain a corresponding expression for the field dependence of $1 / T_{1}$. This allows us to fit 
the data at the lowest temperatures with a phenomenological form that has one additional adjustible parameter corresponding to the spin-dissipation rate. We also present results for the temperature dependence of this effective rate.

Finally, in Section IV we will turn to the regime $T \gg \Delta$. We will do this in the context of the continuum $O(3) \sigma$-model only. Any continuum theory is applicable to real lattice experiments only below some energy scale, and a natural choice for this energy scale is a typical exchange constant $J$. So more specifically, we shall be studying the regime $\Delta \ll T \ll J$. For $T \gg J$ we expect the spins to behave independently, and the system exhibits a Curie susceptibility. It is an open question whether the window of temperatures $\Delta \ll T \ll J$ with universal behavior exists at all in any given system, and the answer will surely depend upon details of the microscopics. It is unlikely to be present for $S=1$ spin chains, but appears quite possible for $S=2$ spin chains [34]. The static properties of this regime were first studied by Joliceour and Golinelli [35] using the $N=\infty$ limit of the $O(N) \sigma$ model of Ref [36]. We shall present here an exact treatment of static and dynamic properties for the case of general $O(N)$; the numerical values of the $N=3$ static results are significantly different from the earlier $N=\infty$ results. We shall show that the antiferromagnetic correlations decay with a correlation length $\xi$, which to leading logarithms in $\Delta / T$ is given at $N=3$ exactly by

$$
\xi=\frac{c}{2 \pi T} \ln \left(\frac{32 \pi e^{-(1+\gamma)} T}{\Delta}\right),
$$

where $\gamma$ is Euler's constant. We also obtain the exact uniform susceptibility

$$
\chi_{u}=\frac{1}{3 \pi c} \ln \left(\frac{32 \pi e^{-(2+\gamma)} T}{\Delta}\right)
$$

(notice the argument of the logarithm differs slightly from (1.8)). It is interesting to compare the two asymptotic results (1.4) and (1.9), and we have done that in Fig 2. It is reassuring to find that the two results are quite compatible for $T \approx \Delta$. This suggests that one of either the $T \ll \Delta$ or $T \gg \Delta$ asymptotics are always appropriate. We shall also consider the nature of spin transport in the $\Delta \ll T \ll J$ regime, and show that it is related to transport in a certain classical statistical problem of deterministic non-linear waves. We have not established whether spin diffusion exists or not in this classical problem; if the correlations were diffusive, however, we are able to precisely predict the $T$ dependence of the spin diffusivity:

$$
D_{s}=\mathcal{B} \frac{T^{1 / 2}[\xi(T)]^{3 / 2}}{\left[3 \chi_{u}(T) / 2\right]^{1 / 2}} .
$$

Here $\mathcal{B}$ is an undetermined universal number, and $\xi(T), \chi_{u}(T)$ are given in (1.8,1.9).

Notice the complementarity in the two $T$ regimes discussed above: the description for $T \ll \Delta$ was in terms of semiclassical particles, while that for $\Delta \ll T \ll J$ is in terms of semiclassical non-linear waves. 


\section{ZERO TEMPERATURE PROPERTIES}

The primary purpose of this section will be to establish the $\mathcal{S}$-matrix by a variety of methods. We will begin in Section $\llbracket \mathrm{A}$ by using the relativistic $O(3) \sigma$ model. In Section ПIB we will consider the strong-coupling expansion of the two-leg ladder in powers of $J_{\|} / J_{\perp}$. This section will also present supplementary results on some interesting features in the $T=0$ INS cross-section of the strongly coupled ladder arising from the presence of a $S=1$, two-particle bound state in its spectrum. Finally, Section $\mathbb{\mathbb { I C }}$ will consider the complementary $J_{\perp} / J_{\|}$ expansion.

\section{A. $O(3) \sigma$ model}

Let us begin with a brief review of the $\sigma$-model as an effective field theory for the low-energy properties of the gapped systems (for a more extensive discussion see [37] and references therein). The imaginary time $(\tau)$ action of the $\sigma$-model is

$$
\mathcal{L}=\frac{c}{2 g} \int_{0}^{1 / T} d \tau d x\left[\left(\partial_{x} n_{\alpha}\right)^{2}+\frac{1}{c^{2}}\left(\partial_{\tau} n_{\alpha}-i \epsilon_{\alpha \beta \gamma} H_{\beta} n_{\gamma}\right)^{2}\right]
$$

where $x$ is the spatial co-ordinate, $\alpha, \beta, \gamma=1,2,3$ are $O(3)$ vector indices over which there is an implied summation, $\epsilon_{\alpha \beta \gamma}$ is the totally antisymmetric tensor, $c$ is a velocity, $H_{\alpha}$ is an external magnetic field (we have absorbed a factor of the electronic magnetic moment, $g_{e} \mu_{B}$, into the definition of the field $H$.), and the partition function is obtained by integrating over the unit vector field $n_{\alpha}(x, \tau)$, with $n_{\alpha}^{2}(x, \tau)=1$. The dimensionless coupling constant $g$ is determined by the underlying lattice antiferromagnet at the momentum scale $\Lambda \sim$ inverse lattice spacing to be $g \sim 1 / S$ where $S$ is the spin at each site in the original lattice system. The $\sigma$-model is used to make statements about physics at length scales $\gg \Lambda^{-1}$ and time scales $\gg(c \Lambda)^{-1}$; this physics is universally characterized by the dimensionful parameters $c, H, T$, and $\Delta$, the energy gap at $T=H=0$. Though the magnitude of $\Delta$ is determined by non-universal lattice scale quantities $\left(\Delta \sim c \Lambda e^{-2 \pi / g}\right.$ for small $\left.g\right)$, the long distance physics of the $\sigma$-model depends on these lattice scale effects only through the value of $\Delta$, and has no direct dependence on $g$ or $\Lambda$. Also, the energy-momentum dispersion of the stable particle-like excitation of this model is given by $\varepsilon(k)=\sqrt{\Delta^{2}+k^{2} c^{2}}$, and there is a triplet of them. The conserved density of this model corresponding to its $O(3)$ symmetry is the magnetization density $M_{\alpha}(x, \tau)=\delta L / \delta H_{\alpha}(x, \tau)$. In the Hamiltonian formalism, this is represented by the operator $M_{\alpha}(x)$.

Finally, to make contact with the lattice antiferromagnet, we must have a prescription for representing the spin-density operator $s_{\alpha}(x)$ of the lattice system in terms of the operators of the $\sigma$-model. It is most convenient to do this in terms of Fourier components. We have:

$$
s_{\alpha}(k+Q) \propto n_{\alpha}(k)
$$

(recall that $Q=\pi / a$ ) and

$$
s_{\alpha}(q)=M_{\alpha}(q)
$$


for $|q|,|k|$ much smaller than some microscopically determined scale $\sim \Lambda$. The missing proportionality constant in the first relation is non-universal and related to the magnitude of the spin at each site in the original lattice system. Thus, the $\sigma$-model allows us to represent spin fluctuations near $q=Q$ (these being the low-energy degrees of freedom) and near $q=0$. This is of course because the $q=0$ component of the spin density is the conserved charge corresponding to the $O(3)$ symmetry of the system, and as such must be included in any description of the slow modes.

The exact $\mathcal{S}$ matrix of the collision of two particles in the $\sigma$-model was computed in a seminal paper of Zamolodchikov and Zamolodchikov. For the scattering event shown in Fig 1 it is (recall $\mu_{i}=x, y, z$ are the three possible values of the $O(3)$ spin label):

$$
\begin{aligned}
\mathcal{S}_{\mu_{1}^{\prime}, \mu_{2}^{\prime}}^{\mu_{1} \mu_{2}}\left(k_{1}, k_{2} ; k_{1}^{\prime}, k_{2}^{\prime}\right)=(2 \pi)^{2} \delta\left(k_{1}-\right. & \left.k_{1}^{\prime}\right) \delta\left(k_{2}-k_{2}^{\prime}\right)\left[\sigma_{1}(\theta) \delta_{\mu_{1} \mu_{2}} \delta_{\mu_{1}^{\prime} \mu_{2}^{\prime}}\right. \\
& \left.+\sigma_{2}(\theta) \delta_{\mu_{1} \mu_{1}^{\prime}} \delta_{\mu_{2} \mu_{2}^{\prime}}+\sigma_{3}(\theta) \delta_{\mu_{1} \mu_{2}^{\prime}} \delta_{\mu_{2} \mu_{1}^{\prime}}\right]
\end{aligned}
$$

where $\theta=\theta_{1}-\theta_{2}$ is the 'rapidity', $k_{i}=(\Delta / c) \sinh \theta_{i}$ for $i=1,2$, and $O(3)$ invariance guarantees a total lack of $H$ dependence in the result. The functions $\sigma$ in (2.2) are

$$
\begin{aligned}
& \sigma_{1}(\theta)=\frac{2 \pi i \theta}{(\theta+\pi i)(\theta-2 \pi i)} \\
& \sigma_{2}(\theta)=\frac{\theta(\theta-\pi i)}{(\theta+\pi i)(\theta-2 \pi i)} \\
& \sigma_{3}(\theta)=\frac{-2 \pi i(\theta-\pi i)}{(\theta+\pi i)(\theta-2 \pi i)}
\end{aligned}
$$

Now notice the structure of the limit $\theta \rightarrow 0$ which is important for our purposes in the region $T \ll \Delta$ : we find that $\sigma_{1,2}(\theta \rightarrow 0)=0$, while $\sigma_{3}(\theta \rightarrow 0)=-1$. This establishes the key result (1.2) for this continuum model.

\section{B. Strongly-coupled two-leg ladders}

In this section we concentrate on the properties of a particular model system, the spin1/2, 2-leg Heisenberg antiferromagnetic ladder, to which the low-energy phenomenology of the preceeding section is expected to apply. The Hamiltonian of the system may be written as

$$
\mathcal{H}=\sum_{i} \vec{S}_{I}(i) \cdot \vec{S}_{I I}(i)+g \sum_{i}\left(\vec{S}_{I}(i) \cdot \vec{S}_{I}(i+1)+\vec{S}_{I I}(i) \cdot \vec{S}_{I I}(i+1)\right)
$$

Here, the $\vec{S}_{I}(i)$ and $\vec{S}_{I I}(i)$ are spin-1/2 operators at site i along the two chains that make up the ladder, $g$ is a dimensionless coupling constant equal to the ratio of the antiferromagnetic bond along the individual chains $J_{\|}$to the bond along the rungs of the ladder $J_{\perp}$ and we have set the bond strength $J_{\perp}$ along the rungs to be unity; this defines our unit of energy. We will analyze this model in the limit of small $g$; this 'strong-coupling' expansion [24] is expected to be qualitatively correct for all $g$. For $g=0$, we just have a system of isolated rungs with the two spins on each rung coupled antiferromagnetically. The ground state is a product 
state with each rung in a singlet state. The lowest lying excited states form a degenerate manifold with precisely one rung promoted to the triplet state. Perturbative corrections in $g$ would presumably make this triplet 'particle' hop around producing a single-particle band of triplet excitations as the lowest lying excited states. Thus we expect that our perturbative analysis will be most conveniently performed in a representation that directly describes the state of individual rungs of the ladder. With this in mind, we switch to the 'bond-operator' formalism introduced in Ref [38]. Following Ref [38], we write the spin operators as:

$$
\begin{aligned}
& S_{I}^{\alpha}(i)=\frac{1}{2}\left(s^{\dagger}(i) t_{\alpha}(i)+t_{\alpha}^{\dagger}(i) s(i)-i \epsilon_{\alpha \beta \gamma} t_{\beta}^{\dagger}(i) t_{\gamma}(i)\right), \\
& S_{I I}^{\alpha}(i)=\frac{1}{2}\left(-s^{\dagger}(i) t_{\alpha}(i)-t_{\alpha}^{\dagger}(i) s(i)-i \epsilon_{\alpha \beta \gamma} t_{\beta}^{\dagger}(i) t_{\gamma}(i)\right),
\end{aligned}
$$

where $\alpha, \beta$, and $\gamma$ are vector indices taking the values $x, y, z$, repeated indices are summed over, and $\epsilon$ is the totally antisymmetric tensor. $s^{\dagger}(i)$ and $t_{\alpha}^{\dagger}(i)$ are respectively creation operators for singlet and triplet bosons at site $i$ (in the previous section we had used $\vec{s}(x)$ to denote the spin density of the lattice system; here we shall use $\vec{\sigma}(x)$ to denote the same and reserve $s$ for the singlet boson operator). The restriction that physical states on a rung are either singlets or triplets leads to the following constraint on the boson occupation numbers at each site:

$$
s^{\dagger}(i) s(i)+t_{\alpha}^{\dagger}(i) t_{\alpha}(i)=1
$$

The spin density is given by

$$
\sigma_{\alpha}(i)=-i \epsilon_{\alpha \beta \gamma} t_{\beta}^{\dagger}(i) t_{\gamma}(i)
$$

It is also convenient to define

$$
\phi_{\alpha}(i)=s^{\dagger}(i) t_{\alpha}(i)+t_{\alpha}^{\dagger}(i) s(i)
$$

The Hamiltonian in terms of these operators is given as

$$
\mathcal{H}=\mathcal{H}_{0}+\mathcal{V}
$$

where

$$
\mathcal{H}_{0}=\sum_{i}\left(-\frac{3}{4} s^{\dagger}(i) s(i)+\frac{1}{4} t_{\alpha}^{\dagger}(i) t_{\alpha}(i)\right)
$$

and

$$
\mathcal{V}=\frac{g}{2} \sum_{i}\left(\phi_{\alpha}(i) \phi_{\alpha}(i+1)-\sigma_{\alpha}(i) \sigma_{\alpha}(i+1)\right)
$$

In this representation, the ground state for $g=0$ is just the state with every site occupied by a singlet boson. To zeroth order in $g$, the lowest excited states form a degenerate manifold with a triplet boson (of arbitrary polarization) replacing the singlet particle at precisely one site. Higher excited states also form degenerate manifolds labelled by the number of singlet 
particles that are replaced by triplet bosons.In what follows, we will describe states by the number (which can only be zero or one) and polarization of the triplet particles at each site, the singlet occupation numbers being determined by the constraint. Thus we will loosely refer to the state with no triplet particles as the 'vacuum'. At this order in $g$, the physical particle-like excitation of the system is created at site $i$ by the action of $\phi_{\alpha}(i)$ on the vacuum, and thus coincides with the bare triplet particle. In general at higher orders in $g$, we expect that the physical single particle states of the system will contain an admixture of states with more than one bare particle present. Similarly, the physical vacuum will also have a component with non-zero bare particle number.

In fact, it is quite convenient to make a canonical transformation (determined order by order in $g$ ) to an auxillary problem in which the physical particle states do not contain admixtures of states with different bare particle number. The Hamiltonian of the auxillary problem is related to the original one by a similarity transformation. The energy eigenvalues obtained in this manner of course give the energy levels of the original Hamiltonian. However, to recover the corresponding wavefunctions, one has to undo the effects of the canonical transformation. We will use this convenient formulation of perturbation theory below as we discuss the strong-coupling expansion.

The auxillary Hamiltonian in this approach is given by

$$
\tilde{\mathcal{H}}=e^{i W} \mathcal{H} e^{-i W}
$$

where $W$ is the hermitian operator that generates our canonical transformation. We choose $W$ to meet the following criteria:

- The matrix elements of $\tilde{\mathcal{H}}$ between states with differing numbers of bare particles should be zero to a given order in $g$. Note that this implies that the elementary excitations of the auxillary Hamiltonian are just the bare particles. However their dynamics, and their mutual interactions (in multiparticle sectors) are determined by the restriction of $\tilde{\mathcal{H}}$ to the appropriate subspace of definite particle number. This restriction gives the corresponding energy levels of the original Hamiltonian $\mathcal{H}$ correct to that order in $g$. This then serves as our effective Hamiltonian for the corresponding sector of the original problem.

- This does not completely specify $W$. We therefore also require that $W$ have zero matrix elements to a given order in $g$ between any two states populated by the same number of bare particles.

These criteria fix $W$ uniquely order by order in $g$ and in general we have an expansion for $W$ that reads: $W=g\left(W_{1}+g W_{2}+\ldots\right)$. It is quite straightforward to use this procedure to generate an expansion in $g$ for the effective Hamiltonians in the one and two particle sector of the original problem (the 'effective Hamiltonian' in the physical vacuum sector is just a constant equal to the ground state energy calculated to the relevant order in $g$ ). Solving for the eigenstates and eigenvalues of these effective Hamiltonians is just a simple exercise in elementary quantum mechanics. If the eigenstates of the spin-ladder are of interest (as they will be when we calculate $S(q, \omega)$ perturbatively), we will have to obtain them from the eigenstates $|\psi\rangle$ of the effective Hamiltonian using 


$$
|\psi\rangle_{\text {physical }}=e^{-i W}|\psi\rangle
$$

After this preamble, we turn to the actual calculations. As we have mentioned earlier, the scattering matrix in the low-energy limit is a crucial input to the semiclassical calculations at non-zero temperature, and it is therefore interesting to have results for it in our microscopic model. So, to begin with, let us look at the two-particle sector and work out the scattering properties of the physical particles.

First we need to find the effective Hamiltonian for the two-particle sector. To first order in $g$, this is just given by the restriction of $\mathcal{H}_{0}+\mathcal{V}$ to the two particle subspace. Instead of introducing a lot of cumbersome notation to write this down, we will just list the amplitudes of the various processes that are allowed in this two body problem:

- Each particle can hop one site to the left or the right with amplitude $g / 2$ except when the neighbouring site in question is occupied by the other particle.

- When the two particles are at neighbouring sites, there is a non-zero amplitude for spin rotation. Consider the state $\left|i, \alpha_{1} ;(i+1), \alpha_{2}\right\rangle$ which has one particle at $i$ with polarization $\alpha_{1}$ (which can be any one of $x, y, z$ ) and another particle at $i+1$ with polarization $\alpha_{2}$. The amplitude to make a transition from this to the state $\left|i, \beta_{1} ;(i+1), \beta_{2}\right\rangle$ is $\left(-g \epsilon_{\gamma \beta_{1} \alpha_{1}} \epsilon_{\gamma \beta_{2} \alpha_{2}}\right) / 2$.

To solve for the scattering states of this two-body problem, it is more convenient to work in a basis in which we label the spin part of the two particle states by the total angular momentum $J$ and the value of its $z$ component $J_{z}$. The spin rotation amplitude now becomes just a $J$ dependent nearest neighbour potential which takes the values $g / 2,-g / 2$, and $-g$ for $J=2,1$, and 0 respectively. Note that the potential energy is independent of $J_{z}$ as one would expect from rotational invariance. It is now quite simple to find the scattering eigenstates in each channel. The spatial wavefunction in channel $J$ may be written as

$$
\psi\left(x_{1}, x_{2}\right)=\hat{P}_{J}\left\{\left(e^{i k_{1} x_{1}+i k_{2} x_{2}}+r_{J}\left(k_{1}, k_{2}\right) e^{i k_{2} x_{1}+i k_{1} x_{2}}\right) \theta\left(x_{2}-x_{1}\right)\right\}
$$

where $\hat{P}_{J}$ is the symmetrizing operator for $J=2,0$ and the antisymmetrizing operator for $J=1$, and $r_{J}$ is the reflection coefficient that completely specifies the scattering properties of the particles. For the $r_{J}$ we have:

$$
\begin{aligned}
& r_{2}=-\frac{e^{-i k a}-2 \cos \left(k_{c m} a / 2\right)}{e^{i k a}-2 \cos \left(k_{c m} a / 2\right)}, \\
& r_{1}=-\frac{e^{-i k a}+2 \cos \left(k_{c m} a / 2\right)}{e^{i k a}+2 \cos \left(k_{c m} a / 2\right)}, \\
& r_{0}=-\frac{e^{-i k a}+\cos \left(k_{c m} a / 2\right)}{e^{i k a}+\cos \left(k_{c m} a / 2\right)} ;
\end{aligned}
$$

where $k=\left(k_{1}-k_{2}\right) / 2, k_{c m}=k_{1}+k_{2}$, and $a$ is the lattice spacing along the length of either of the two chains that make up the ladder system. Note that $k_{1}$ and $k_{2}$ both range over the interval $(0,2 \pi / a)$. The energy of the scattering state labelled by $\left\{k_{1}, k_{2}\right\}$ (the energy of the ground state being set to zero) is given by 


$$
E\left(k_{1}, k_{2}\right)=2+g \cos \left(k_{1} a\right)+g \cos \left(k_{2} a\right) .
$$

This is consistent with the first order result [24 for the single particle dispersion relation: $E(k)=1+g \cos (k a)$.

The next step is to use these results for the reflection coefficients to obtain the $\mathcal{S}$-matrix for this two-body problem in the limit of low velocities. Low velocities imply values of $k_{1}$ and $k_{2}$ in the vicinity of the band minimum at $\pi / a$, i.e $k$ close to zero and $k_{c m}$ close to $2 \pi / a$. Both $r_{2}$ and $r_{1}$ have the limiting value -1 as $k \rightarrow 0, k_{c m} \rightarrow 2 \pi / a$. However, $r_{0}$ is singular in the vicinity of $k=0, k_{c m}=2 \pi / a$; its value depends on the order in which the two limits $k \rightarrow 0$ and $k_{c m} \rightarrow 2 \pi / a$ are taken. This is somewhat disconcerting as we expect a well-defined low-velocity limit which agrees with the predictions of the $O(3) \mathrm{NL} \sigma \mathrm{M}$ field theory.

To identify the source of our problem, let us look more closely at the expression for $r_{0}$. We notice that $r_{0}$, considered a function of the complex variable $k$, has a pole in the upper halfplane for a range of values of $k_{c m}$. This indicates the presence of a bound state in the $J=0$ channel for the corresponding values of $k_{c m}$. This bound state hits threshold, i.e its binding energy goes to zero, as $k_{c m} \rightarrow 2 \pi / a$. It is the presence of a bound state at threshold that causes the singular behaviour of the reflection coefficient in the limit $k \rightarrow 0, k_{c m} \rightarrow 2 \pi / a$. Clearly, if there were a range of $k_{c m}$ around $2 \pi / a$ for which there was no singlet bound state, then we would not have this difficulty. It turns out (as we shall briefly outline later) that extending our calculation to the next order in $g$ leads us to precisely this conclusion and gives a well-defined limiting value of -1 for $r_{0}$ as $k \rightarrow 0, k_{c m} \rightarrow 2 \pi / a$.

This result can now be used to obtain the $\mathcal{S}$-matrix of our auxillary two-body problem. We are interested, however, in the $\mathcal{S}$-matrix that describes the scattering of the physical particle-like excitations of the spin-ladder. Thankfully, it is quite easy to see that though the wavefunctions of the two problems are related by a canonical transformation, the purely 'off-diagonal' form of $W$ implies that the two are the same at least to first order in $g$. Transforming to the basis used in (1.2), we see that the $\mathcal{S}$-matrix in the low velocity limit is indeed given by (1.2) Thus, this super-universal form of the $\mathcal{S}$-matrix holds for our lattice model and lends support to the idea that it is a generally valid consequence of just the slow motion of the particles and is in no way dependent on the special properties of the continuum $\sigma$-model.

To wind up this part of our discussion, let us now summarize the calculation of the reflection coefficients to first order in $g$. We need to find the effective Hamiltonian of our auxillary two-body problem to second order in $g$. This involves first working out $W_{1}$ and then using this to obtain the effective two-body Hamiltonian. To $\mathcal{O}\left(g^{2}\right)$, we generate in this manner an additional next-nearest neighbour hopping term and some additional $J$ dependent nearest-neighbour interactions. We skip the details as they are somewhat tedious and not particularly illuminating. The $J=0$ reflection coefficient (correct to $\mathcal{O}(g)$ ) obtained in this manner is given as:

$$
r_{0}=-\frac{e^{-i k a}+\cos \left(k_{c m} a / 2\right)-g \cos \left(k_{c m} a\right)\left(3 e^{-i k a}+e^{i k a}\right) / 4}{e^{i k a}+\cos \left(k_{c m} a / 2\right)-g \cos \left(k_{c m} a\right)\left(3 e^{i k a}+e^{-i k a}\right) / 4} .
$$

From this, it is easy to see that there is no pole in the upper half $k$-plane as long as $\left|k_{c m} a-2 \pi\right|<\sqrt{8 g}$. This means that there is no singlet bound state possible in this range of 
$k_{c m}$. This is consistent with our expectation that at the very lowest energies, the two-particle spectrum should be free of bound states in order to match the predictions of the $\sigma$-model. Moreover, (2.16) has a well-defined low-velocity limit of -1 as claimed earlier.

The foregoing analysis has shown that the two particle sector has a spin $S=0$ bound state which leads to some interesting threshold singularities for the scattering matrix. Examining our expressions for the reflection coefficients, we notice that there is in fact a bound state in the $S=1$ channel as well (actually, there is also a $S=2$ anti-bound state; we will not delve further into that aspect of the spectrum here). Now, a $S=1$ excited state can have observable consequences for the INS cross-section of a system and we might expect to see some interesting features in the same as a result of this.

With this motivation, let us turn to the perturbative calculation of the dynamic structure factor at $T=0$. We pick a coordinate system in which the two chains that make up our ladder are parallel to the $x$ axis and have $y$ coordinates of $+d / 2$ and $-d / 2$ respectively, where $d$ is the distance between them (for simplicity, we are assuming here that the rungs of the ladder are perpendicular to its legs). The spins along a chain are located at $x$ values equal to integer multiples of $a$. We denote the position of each spin in the $x-y$ plane by $\vec{R}$. We define $\vec{P}=\left(q_{x}, q_{y}\right)$. The $T=0$ dynamic structure factor may be written as

$$
S(\vec{P}, \omega)=\frac{1}{2 \pi} \int d t \frac{a}{2 L} \sum_{\vec{R} \vec{R}^{\prime}}\left\langle\Phi_{0}\left|\hat{S}_{\vec{R}}^{z}(t) \hat{S}_{\vec{R}^{\prime}}^{z}(0)\right| \Phi_{0}\right\rangle e^{-i \vec{P} \cdot\left(\vec{R}-\vec{R}^{\prime}\right)+i \omega t} ;
$$

where $\left|\Phi_{0}\right\rangle$ is the ground state of the system, $L$ is the length of each chain, and $\hat{S}_{\vec{R}}$ denotes the spin operator at $\vec{R}$ in the Heisenberg representation. Our strategy is to write down the usual spectral representation for (2.17) and then evaluate it perturbatively. Actually, a complete calculation of the second order contribution would involve the eigenstates with more than two-particles present; below we will ignore this complication and confine ourselves to calculating the contribution of the one and two-particle sectors, correct to the appropriate order in $g$.

The spin operator at any site is a sum of two terms: a single particle piece coming from the $\phi_{\alpha}$, and a two particle part coming from the spin density operator $\sigma_{\alpha}$. From the structure of the strong-coupling expansion, it is clear that the single particle part does not have matrix elements between the ground state and any state in the two particle sector; similarly the two-particle piece does not have matrix elements between the ground state and any state in the single-particle sector. Thus, keeping only the contributions from the one and two particle sectors, we can write to second order in $g$ :

$$
\begin{aligned}
S(\vec{P}, \omega)= & \frac{1}{2} \sum_{1-\text { particle states }} \delta\left(\omega-E_{1}\right)\left|\left\langle\Phi_{1}\left|\phi_{z}\left(-q_{x}\right)\right| \Phi_{0}\right\rangle\right|^{2} \sin ^{2}\left(\frac{q_{y} d}{2}\right) \\
& +\frac{1}{2} \sum_{2-\text { particle states }} \delta\left(\omega-E_{2}\right)\left|\left\langle\Phi_{2}\left|\sigma_{z}\left(-q_{x}\right)\right| \Phi_{0}\right\rangle\right|^{2} \cos ^{2}\left(\frac{q_{y} d}{2}\right) ;
\end{aligned}
$$

where $\left|\Phi_{1}\right\rangle$ and $\left|\Phi_{2}\right\rangle$ denote one and two particle states respectively, and $\phi_{z}\left(q_{x}\right)$ and $\sigma_{z}\left(q_{x}\right)$ denote the discrete Fourier transforms of $\phi_{z}(x)$ and $\sigma_{z}(x)$. Let us digress for a moment and think in terms of the inelastic neutron scattering cross-section for a process with momentum transfer $\vec{P}$ and energy tranfer $\omega$; this coincides with the dynamic structure factor apart from 
some geometrical factors. This scattering can of course produce a single spin-one particle in the spin system. But there is also a non-zero amplitude for producing a pair of these particles close to each other (as is clear from the actual calculations described later). This is the origin of the second term in (2.18).

Now, these two pieces contribute to the structure factor over very distinct intervals along the frequency axis. While it is in principle possible to calculate both terms correct to $\mathcal{O}\left(g^{2}\right)$, we will confine ourselves below to calculating the leading perturbative correction for each value of $\omega$. Thus, we will calculate the single particle piece only to first order in $g$, while doing a full second order calculation for the two-particle piece. Below, we give a brief outline of the calculation and then discuss our final results.

To calculate the single particle piece, we first need to determine the ground state and the physical one-particle state wavefunctions correct to $\mathcal{O}(g)$. This involves using $W$ correct to first order to obtain the physical wavefunctions from the wavefunctions of the corresponding auxillary problem (for the one-particle sector, these are just plane waves to all orders in $g$; this follows from translational invariance). A simple calculation then gives the one-particle piece as

$$
S_{1}(\vec{P}, \omega)=\frac{1}{2}\left(1-g \cos \left(q_{x} a\right)\right) \sin ^{2}\left(\frac{q_{y} d}{2}\right) \delta\left(\omega-E\left(q_{x}\right)\right),
$$

where $E\left(q_{x}\right)=1+g \cos \left(q_{x} a\right)$.

Turning to the two-particle piece, we see that one can actually ignore the distinction between the physical 2-particle wavefunction and the wavefunction of the auxillary two-body problem. Moreover, it suffices to consider the auxillary problem to first order in $g$. Also, since the ground state has spin zero and we are looking at the matrix elements of a vector operator, we need to consider only the triplet $(J=1)$ channel of the auxillary problem. The only subtlety lies in the fact that we need to consider the bound state contribution as well as the usual contribution of the scattering states. From (2.14), we see that this bound state exists for $\pi<k_{c m} a<\pi+\pi / 3$ and for $3 \pi-\pi / 3<k_{c m} a<3 \pi$ (remember $k_{c m}$ ranges from 0 to $\left.4 \pi / a\right)$. Thinking in terms of an inelastic neutron scattering event with momentum transfer $q_{x}$ in the fundamental domain $(0,2 \pi / a)$, we see that this bound state can be excited for $\pi<q_{x} a<\pi+\pi / 3$ and for $\pi-\pi / 3<q_{x} a<\pi$. In the latter case, momentum is conserved modulo a reciprocal lattice vector of $2 \pi / a$. Of course, in addition to the bound state contribution there is a background term coming from the scattering states in this channel. Again, the two particles can be created in the scattering state either with total momentum $k_{c m}$ equal to the momentum transfer $q_{x}$, or with the two differing by a reciprocal lattice vector of $2 \pi / a$.

The actual calculations are quite elementary and we proceed directly to the results for the two-particle contributions. The bound state contribution for $2 \pi / 3<q_{x} a<4 \pi / 3$ may be written as:

$$
S_{B}(\vec{P}, \omega)=\frac{g^{2}}{2} \cos ^{2}\left(\frac{q_{y} d}{2}\right) \sin ^{2}\left(\frac{q_{x} a}{2}\right)\left(1-4 \cos ^{2}\left(\frac{q_{x} a}{2}\right)\right) \delta\left(\omega-E_{B}\left(q_{x}\right)\right),
$$

where $E_{B}\left(q_{x}\right)=2-g\left(1+4 \cos ^{2}\left(q_{x} a / 2\right)\right) / 2$. On the other hand, the scattering states give rise to the following background contribution for $|\omega-2|<+2 g\left|\cos \left(q_{x} a / 2\right)\right|$ : 


$$
S_{s c}(\vec{P}, \omega)=\frac{g}{\pi} \cos ^{2}\left(\frac{q_{y} d}{2}\right) \sin ^{2}\left(\frac{q_{x} a}{2}\right) \frac{\sqrt{4 g^{2} \cos ^{2}\left(q_{x} a / 2\right)-(\omega-2)^{2}}}{g+2(\omega-2)+4 g \cos ^{2}\left(q_{x} a / 2\right)} .
$$

Note that for $q_{x} a=2 \pi / 3$ or $4 \pi / 3$, there is a square-root divergence at the lower threshold to the continuum in $\omega$; these are precisely the values of $q_{x}$ for which the binding energy of the triplet bound-state goes to zero. This enhanced scattering can thus be thought of as arising from the presence of the triplet bound state at threshold. The salient features of these results are summarized in Fig 3 and Fig 4 . Fig 3 is a plot of the positions along the $\omega$ axis of the single particle peak, the bound state peak, and the bottom of the two-particle continuum as a function of $q_{x}$. In Fig 4, we show the spectral weight in the single particle and bound state peaks as a function of $q_{x}$.

Thus, we see that that the existence of a triplet bound state of two elementary spin-one excitations leads to some interesting features in the dynamic structure factor. Actually, qualitatively similar features, again arising from a triplet bound-state, had been predicted earlier [39] in the alternating one-dimensional Heisenberg antiferromangnetic chain. Recent INS experiments [40,41] on $(\mathrm{VO})_{2} \mathrm{P}_{2} \mathrm{O}_{7}$ do indeed see a second sharply defined peak in the dynamical structure factor for a range of values of $q_{x}$. While this compound had been previously thought to be a good example of a spin-ladder [42], more recent work [43] has favoured the alternating chain model [44] and the INS results have been interpreted [41] in terms of the additional bound state contribution predicted in Ref [39]. Thus, our results may not be of direct relevance to this particular experimental system. However, our work does predict that a second peak in the INS cross-section should be seen in strongly coupled ladder systems and it is quite possible that the feature persists to all orders in the perturbation expansion we have employed. It would be interesting to confirm this effect by looking at other systems that are more convincingly modelled by a simple ladder Hamiltonian and it is hoped that future experiments do indeed see the effects coming from the bound state.

\section{Weakly-coupled two-leg ladders}

In this section, we analyze the ladder system (2.4) in the complementary weak-coupling limit: $J_{\perp} \ll J_{\|}$. An elegant mapping developed by Shelton et.al. [25] allows one to express the low-energy, long-distance properties of the model in terms of a continuum theory of weakly-interacting massive Majorana (real) fermions. We will analyze the low-energy scattering properties of the spin one excitations of the weakly-coupled ladder by working in this Majorana fermion representation.

We begin with a brief review of the Majorana fermion representation. We will not attempt here to describe in any detail the procedure used [25] to arrive at this field-theoretic representation. Instead, we will be content with a rather telegraphic summary of the principal steps involved. To begin with, one writes down the usual, free, massless bosonic theory [14] for the low-energy properties of each of the two $S=1 / 2$ Heisenberg antiferromagnetic chains that make up the ladder. The interchain exchange $J_{\perp}$ is then turned on, introducing a local, isotropic (in spin space) coupling between the spin-density operators of each chain in the bosonic representation. This has two pieces to it: one coupling the staggered parts of the spin densities with each other and the other doing the same for the 
uniform component. Now, one works with symmetric and antisymmetric combinations of the two boson fields (one for each chain) and transcribes everything to a fermionic representation, introducing one Dirac fermion for the symmetric combination and another for the antisymmetric combination in the usual manner (for a readable account of the relevant machinery of Abelian bosonization, see for instance the review [45] by Shankar). The last step is to write each Dirac fermion as two Majorana fermions. If one leaves out the uniform part of the coupling to begin with, the theory in terms of the Majorana fermions is, remarkably enough, a free-field theory. The staggered part of the coupling just provides a mass $\Delta$ to each of the two Majorana fermions obtained from the symmetric combination of the bosons, while the two Majorana fermions obtained from the antisymmetric combination acquire masses $\Delta$ and $-3 \Delta$ respectively (the actual energy gap is given by the absolute value of the mass). The three Majorana fermions with mass $\Delta$ form the spin one triplet we expect on general grounds, and the fourth Majorana fermion represents a high-energy singlet mode that will not be very important for our purposes. The mass parameter $\Delta$ of the theory is proportional to $J_{\perp}$ with the proportionality constant being non-universal. Finally, turning on the coupling between the uniform part of the spin densities gives us a four-fermion interaction term between these massive Majorana fermions which will play a crucial role in our analysis of the $\mathcal{S}$-matrix.

The procedure outlined above gives us the following Hamiltonian for the effective field theory written in terms of Majorana fermions:

$$
\mathcal{H}=\sum_{a=x, y, z} \mathcal{H}_{\Delta}\left(\xi^{a}\right)+\mathcal{H}_{-3 \Delta}(\rho)+\mathcal{H}_{I}
$$

here the $\xi^{a}$ and $\rho$ are Majorana fermion fields with anticommutation relations given as

$$
\begin{aligned}
& \left\{\rho_{R}(x), \rho_{R}(y)\right\}=\delta(x-y) \\
& \left\{\rho_{L}(x), \rho_{L}(y)\right\}=\delta(x-y) \\
& \left\{\xi_{R}^{a}(x), \xi_{R}^{b}(y)\right\}=\delta_{a b} \delta(x-y) \\
& \left\{\xi_{L}^{a}(x), \xi_{L}^{b}(y)\right\}=\delta_{a b} \delta(x-y)
\end{aligned}
$$

with all other anticommutators being equal to zero, $\mathcal{H}_{m}(\phi)$ is defined in general as

$$
\mathcal{H}_{m}(\phi)=\frac{i v_{F}}{2} \int d x\left(\phi_{L} \partial_{x} \phi_{L}-\phi_{R} \partial_{x} \phi_{R}-m \phi_{R} \phi_{L}\right)
$$

with $v_{F} \sim J_{\|} a$ and the interaction term $\mathcal{H}_{I}$ may be written as

$$
\mathcal{H}_{I}=g \int d x\left\{\xi_{R}^{x} \xi_{L}^{x} \xi_{R}^{y} \xi_{L}^{y}+\xi_{R}^{y} \xi_{L}^{y} \xi_{R}^{z} \xi_{L}^{z}+\xi_{R}^{z} \xi_{L}^{z} \xi_{R}^{x} \xi_{L}^{x}-\left(\xi_{R}^{x} \xi_{L}^{x}+\xi_{R}^{y} \xi_{L}^{y}+\xi_{R}^{z} \xi_{L}^{z}\right) \rho_{R} \rho_{L}\right\}
$$

with $g \sim J_{\perp} a$. Note that each Majorana fermion is a two component object, the two components being labelled with the subscripts $R$ and $L$ to denote the 'right-moving' and 'leftmoving' parts. To make contact with the original spin-ladder, we also need a prescription for expressing the spin-operators of the ladder in terms of the Majorana fermions. In sharp contrast to the $\sigma$-model, only the uniform part of the spin-density operator has a local representation in terms of the fermions; the components of the spin-density near $q=Q$ can 
be expressed only in terms of highly non-local functions of the fermi-fields 25. We have the following expressions [25] for the uniform parts, $\vec{J}_{1}$ and $\vec{J}_{2}$, of the spin density on each chain:

$$
\begin{aligned}
J_{1}^{a}(x) & =\frac{i}{2}\left(\frac{1}{2} \epsilon^{a b c} \xi_{\nu}^{b}(x) \xi_{\nu}^{c}(x)+\xi_{\nu}^{a}(x) \rho_{\nu}(x)\right), \\
J_{2}^{a}(x) & =\frac{i}{2}\left(\frac{1}{2} \epsilon^{a b c} \xi_{\nu}^{b}(x) \xi_{\nu}^{c}(x)-\xi_{\nu}^{a}(x) \rho_{\nu}(x)\right),
\end{aligned}
$$

where the index $\nu$ takes on values $R$ or $L$ and repeated indices are summed over. Note that the field $\rho$ corresponding to the non-universal high-energy singlet mode drops out of the expression for the uniform part of the total spin-density of the ladder which can then be expressed entirely in terms of the spin one triplet fields.

We shall find it convenient, when it comes to actually doing any calculations, to rewrite all of the foregoing in terms of fermionic creation and annihilation operators. These are defined as follows: Let $\hat{\xi}_{\nu}^{a}(p)$ and $\hat{\rho}_{\nu}(p)$ denote the Fourier transforms of $\xi_{\nu}^{a}(x)$ and $\rho_{\nu}(x)$ respectively. We write

$$
\begin{aligned}
& \hat{\xi}_{\nu}^{a}(p)=f_{\nu}(p) t_{a}(p)+\bar{f}_{\nu}(-p) t_{a}^{\dagger}(-p), \\
& \hat{\rho}_{\nu}(p)=g_{\nu}(p) s(p)+\bar{g}_{\nu}(-p) s^{\dagger}(-p),
\end{aligned}
$$

where $t_{a}(p)$ and $s(p)$ are the fermionic annihilation operators corresponding to the triplet and singlet modes respectively and $f_{\nu}(p)$ and $g_{\nu}(p)$ are complex-valued functions of $p$ which we specify below. These creation and annihilation operators obey the usual anticommutation relations:

$$
\begin{aligned}
\left\{t_{a}(p), t_{b}^{\dagger}(q)\right\} & =2 \pi \delta_{a b} \delta(p-q) \\
\left\{s(p), s^{\dagger}(q)\right\} & =2 \pi \delta(p-q)
\end{aligned}
$$

with all other anticommutators equal to zero. In terms of these operators, the non-interacting part of the Hamiltonian reads

$$
\mathcal{H}_{0}=\int_{-\infty}^{\infty} \frac{d p}{2 \pi} \varepsilon(p) t_{a}^{\dagger}(p) t_{a}(p)+\int_{-\infty}^{\infty} \frac{d p}{2 \pi} \varepsilon_{s}(p) s^{\dagger}(p) s(p),
$$

where $\varepsilon(p)=\left(p^{2} v_{F}^{2}+\Delta^{2}\right)^{1 / 2}, \varepsilon_{s}(p)=\left(p^{2} v_{F}^{2}+9 \Delta^{2}\right)^{1 / 2}$, and the repeated index $a$ is summed over. The functions $f_{\nu}$ and $g_{\nu}$ are actually chosen to ensure that the non-interacting Hamiltonian has this simple diagonal form in terms of the creation and annihilation operators; this choice guarantees that the operators $s^{\dagger}$ and $t_{a}^{\dagger}$, as defined in (2.27), create the true quasiparticles of the non-interacting system. The expressions for $f_{\nu}$ and $g_{\nu}$ are best written as follows:

$$
\begin{aligned}
& f_{R}(p)=u_{\Delta}(p) \quad p>0, \\
& f_{R}(p)=i v_{\Delta}(p) \quad p<0, \\
& f_{L}(p)=\bar{f}_{R}(-p) \quad \forall p, \\
& g_{R}(p)=u_{(-3 \Delta)}(p) \quad p>0, \\
& g_{R}(p)=i v_{(-3 \Delta)}(p) \quad p<0, \\
& g_{L}(p)=\bar{g}_{R}(-p) \quad \forall p ;
\end{aligned}
$$


here the functions $u_{m}(p)$ and $v_{m}(p)$ are defined in general as

$$
\begin{aligned}
& u_{m}(p)=\cos \left(\theta_{m}(p) / 2\right) \\
& v_{m}(p)=\sin \left(\theta_{m}(p) / 2\right)
\end{aligned}
$$

with the angle $\theta_{m}(p)$ being specified by $\cos \left(\theta_{m}(p)\right)=v_{F}|p| /\left(m^{2}+v_{F}^{2} p^{2}\right)^{1 / 2}, \sin \left(\theta_{m}(p)\right)=$ $m \operatorname{sgn}(p) /\left(m^{2}+v_{F}^{2} p^{2}\right)^{1 / 2}$. Now, we can rewrite the interaction term in normal ordered form with respect to these singlet and triplet creation and annihilation operators. The quadratic terms so generated give the first order correction to the masses of the singlet and triplet modes (this correction has already been calculated in Ref [25] by other means). The quartic term left over, has, in addition to the usual, normal-ordered, particle-number conserving piece, other pieces that involve pair creation and destruction. The full expressions are somewhat messy and we refrain from displaying them here. However, and this is key, we will need only a very simple part (corresponding to the low momentum limit of the particle-number conserving piece) of this quartic term for the calculation of the $\mathcal{S}$-matrix in the low-momentum limit; our method of writing everything in terms of the creation and annihilation operators has the advantage of identifying and isolating this piece at the very outset. Finally, as an aside, we note that the total spin operator of the system may be written in terms of the triplet operators as

$$
S_{\text {tot }}^{a}=i \epsilon^{a b c} \int_{-\infty}^{\infty} \frac{d p}{2 \pi} t_{b}^{\dagger}(p) t_{c}(p)
$$

this confirms that the triplet creation operator $t_{a}^{\dagger}$ does indeed create a single spin one quasiparticle (with polarization $a$ ) of the non-interacting system.

With all of this in mind, let us turn to the analysis of the scattering properties of this model. As we are hoping to calculate the $\mathcal{S}$-matrix perturbatively in the coupling $g$, it is convenient to write $\mathcal{S}=\mathbf{1}+i \mathcal{T}$. The 'transition-matrix' $\mathcal{T}$ can then be calculated perturbatively using the standard field-theoretic prescription that relates it to the corresponding amputated, connected Green's functions of the theory. Let us make this precise for the case we are interested in: namely, a scattering process in which the initial state consists of two particles, one with momentum $k_{1}$ and spin polarization $\mu_{1}$, and the other with momentum $k_{2}$ and spin polarization $\mu_{2}$, and the final state has two particles labelled by $\left(k_{1}^{\prime}, \mu_{1}^{\prime}\right)$ and $\left(k_{2}^{\prime}, \mu_{2}^{\prime}\right)$. Note that we are now not talking about the bare particles of the non-interacting theory, but the actual physical quasiparticle states of the system, correct to the relevant order in the perturbative expansion in $g$. The corresponding matrix element, $\mathcal{S}_{\mu_{1}^{\prime}, \mu_{2}^{\prime}}^{\mu_{1} \mu_{2}}\left(k_{1}, k_{2} ; k_{1}^{\prime}, k_{2}^{\prime}\right) \equiv\left\langle k_{1}^{\prime} \mu_{1}^{\prime}, k_{2}^{\prime} \mu_{2}^{\prime}|\mathcal{S}| k_{1} \mu_{1}, k_{2} \mu_{2}\right\rangle$, may then be written as

$$
\begin{array}{r}
\mathcal{S}_{\mu_{1}^{\prime}, \mu_{2}^{\prime}}^{\mu_{1} \mu_{2}}\left(k_{1}, k_{2} ; k_{1}^{\prime}, k_{2}^{\prime}\right)=(2 \pi)^{2} \delta_{\mu_{1} \mu_{1}^{\prime}} \delta_{\mu_{2} \mu_{2}^{\prime}} \delta\left(k_{1}-k_{1}^{\prime}\right) \delta\left(k_{2}-k_{2}^{\prime}\right)+ \\
(2 \pi)^{2} \delta\left(E_{f}-E_{i}\right) \delta\left(k_{f}-k_{i}\right) i \mathcal{M}_{\mu_{1}^{\prime}, \mu_{2}^{\prime}}^{\mu_{1} \mu_{2}}\left(k_{1}, k_{2} ; k_{1}^{\prime}, k_{2}^{\prime}\right),
\end{array}
$$

where $E_{f}=\varepsilon\left(k_{1}^{\prime}\right)+\varepsilon\left(k_{2}^{\prime}\right)$ and $E_{i}=\varepsilon\left(k_{1}\right)+\varepsilon\left(k_{2}\right)$ are the final and initial energies respectively, $k_{f}$ and $k_{i}$ are the total momenta in the final and initial states respectively, and $\mathcal{M}$ is the 'reduced' matrix element (with energy and momentum conserving $\delta$ functions removed) for the process under consideration. 
We now specialize to the case $k_{1}=k, k_{2}=-k(k>0)$; this special case allows us to make our basic point (regarding the infrared divergences present in a perturbative calculation of the scattering properties) while keeping the calculations simple. In this case, we may decompose the scattering matrix as follows:

$$
\begin{aligned}
\mathcal{S}_{\mu_{1}^{\prime}, \mu_{2}^{\prime}}^{\mu_{1} \mu_{2}}\left(k,-k ; k_{1}^{\prime}, k_{2}^{\prime}\right)=\delta\left(k_{1}^{\prime}-k\right) \delta( & \left.k_{2}^{\prime}+k\right)\left[S_{1}(k) \delta_{\mu_{1} \mu_{2}} \delta_{\mu_{1}^{\prime} \mu_{2}^{\prime}}\right. \\
+ & \left.S_{2}(k) \delta_{\mu_{1} \mu_{1}^{\prime}} \delta_{\mu_{2} \mu_{2}^{\prime}}+S_{3}(k) \delta_{\mu_{1} \mu_{2}^{\prime}} \delta_{\mu_{2} \mu_{1}^{\prime}}\right] .
\end{aligned}
$$

Now, energy and momentum conservation in one dimension provide enough constraints on the two-body problem to ensure that the allowed final states have the same set of momentum labels as the initial state. This allows us to convert the overall energy and momentum conserving $\delta$ functions in the second term of (2.33) to $\delta$ functions that identify $k_{1}^{\prime}$ with $k$ and $k_{2}^{\prime}$ with $-k$. In the process, we of course introduce additional kinematic factors coming from the Jacobian (we are basically using $\delta(f(x))=\delta(x) /\left|f^{\prime}(x)\right|$ ). Using this, we can write

$$
\begin{aligned}
& S_{1}(k)=\left(\frac{\varepsilon(k)}{2 k v_{F}^{2}}\right) i M_{1}(k), \\
& S_{2}(k)=1+\left(\frac{\varepsilon(k)}{2 k v_{F}^{2}}\right) i M_{2}(k), \\
& S_{3}(k)=\left(\frac{\varepsilon(k)}{2 k v_{F}^{2}}\right) i M_{3}(k),
\end{aligned}
$$

where $M_{1}, M_{2}$, and $M_{3}$ are defined in terms of the following decomposition for $\mathcal{M}$ :

$$
\mathcal{M}_{\mu_{1}^{\prime}, \mu_{2}^{\prime}}^{\mu_{1} \mu_{2}}(k,-k ; k,-k)=\left[M_{1}(k) \delta_{\mu_{1} \mu_{2}} \delta_{\mu_{1}^{\prime} \mu_{2}^{\prime}}+M_{2}(k) \delta_{\mu_{1} \mu_{1}^{\prime}} \delta_{\mu_{2} \mu_{2}^{\prime}}+M_{3}(k) \delta_{\mu_{1} \mu_{2}^{\prime}} \delta_{\mu_{2} \mu_{1}^{\prime}}\right] \text {. }
$$

The relations (2.35) are useful because there is a simple diagrammatic prescription for the perturbative evaluation of $\mathcal{M}$. According to this standard field theoretic prescription [46], $i \mathcal{M}_{\mu_{1}^{\prime}, \mu_{2}^{\prime}}^{\mu_{1}^{\prime} \mu_{2}}\left(k_{1}, k_{2} ; k_{1}^{\prime}, k_{2}^{\prime}\right)$ is proportional to the sum of all 'amputated' (factors corresponding to external legs omitted), fully connected, one particle irreducible diagrams contributing to the time ordered four-point function with two incoming external lines and two outgoing external lines. The incoming lines must carry momenta $k_{1}$ and $k_{2}$, frequencies $\omega_{1}$ and $\omega_{2}$ set to their respective 'on-shell' values of $\varepsilon\left(k_{1}\right)$ and $\varepsilon\left(k_{2}\right)$, and spin labels $\mu_{1}$ and $\mu_{2}$ respectively. The outgoing lines must carry momenta $k_{1}^{\prime}$ and $k_{2}^{\prime}$, frequencies again set to their on-shell values of $\varepsilon\left(k_{1}^{\prime}\right)$ and $\varepsilon\left(k_{2}^{\prime}\right)$, and spin labels $\mu_{1}^{\prime}$ and $\mu_{2}^{\prime}$ respectively. Denoting the sum of all such diagrams schematically by $\Gamma_{4}$, we can write

$$
i \mathcal{M}_{\mu_{1}^{\prime}, \mu_{2}^{\prime}}^{\mu_{1} \mu_{2}}\left(k_{1}, k_{2} ; k_{1}^{\prime}, k_{2}^{\prime}\right)=(\sqrt{Z})^{4} \Gamma_{4}\left(k_{1} \mu_{1}, k_{2} \mu_{2} ; k_{1}^{\prime} \mu_{1}^{\prime}, k_{2}^{\prime} \mu_{2}^{\prime}\right),
$$

where the field-strength renormalization factor $Z$ comes into play because the singlet and triplet creation operators $s^{\dagger}$ and $t_{a}^{\dagger}$ create the bare particles, while we are asking questions about the scattering properties of the physical quasiparticle excitations. We will not be very careful here about the precise definition of $Z$; it will soon become apparent that this does not play any role in the calculation we do.

Before we set about calculating $\Gamma_{4}$, we need to specify our conventions regarding the diagrammatic representation of perturbation theory. As shown in Fig 5, we denote the 
propogator of the triplet particle by a solid line with an arrow carrying momentum $k$, frequency $\omega$ and spin label $\mu$; this has a factor of $i /(\omega-\varepsilon(k)+i \eta)$ associated with it. It turns out that we do not need to consider any diagrams that have lines corresponding to singlet particles and we will not bother to introduce a diagrammatic representation for their propogator. We also display our diagram convention for the four point vertices of the theory in the same figure; again, only the particle number conserving vertices in which all four lines involved correspond to triplet particles have been assigned a diagram as the others will not play a role in what follows. One type of vertex, labelled (a) in Fig 5 , depicts a process in which two particles of momentum $p_{3}$ and $p_{4}$, both with spin label $\mu=x$ scatter into a final state populated by two particles with momenta $p_{1}$ and $p_{2}$, and spin label $\mu=y$. The full momentum dependent factor associated with this diagram is also shown below it. We will need only a very simple low-momentum limit of this expression in most of what follows. The other kind of vertex, labelled (b) in Fig 5, shows incoming particles with labels $\left(p_{3} y\right)$ and $\left(p_{4} x\right)$ scattering into a final state populated by particles with labels $\left(p_{1} x\right)$ and $\left(\begin{array}{ll}p_{2} & y\end{array}\right)$ respectively. Again, the full momentum dependent factor is displayed alongside for completeness. We will mostly need only the value of this factor when all four momenta equal zero; this is given simply by $-i g$. Of course, all other vertices of the same type, but having different spin labels that can be obtained from these using the $O(3)$ symmetry of the problem, have the same factors associated with them.

We are now in a postition to do some calculations. We begin by noting that, apart from the overall factor of $(\sqrt{Z})^{4}$ which we are ignoring for now, $i M_{1}(k), i M_{2}(k)$ and $i M_{3}(k)$ are equal to $\Gamma_{4}(k x,-k x ; k y,-k y), \Gamma_{4}(k x,-k y ; k x,-k y)$, and $\Gamma_{4}(k x,-k y ; k y,-k x)$ respectively. It is quite simple to calculate these three quantities to leading order in $g$. The diagrams contributing to $i M_{1}$ is shown in Fig 6, while those contributing to $i M_{2}$ and $i M_{3}$ are shown in Fig 1 . Evaluating these 'tree-level' amplitudes, we obtain

$$
\begin{aligned}
& \Gamma_{4}(k x,-k x ; k y,-k y)=i g \frac{k^{2} v_{F}^{2}}{\varepsilon^{2}(k)}, \\
& \Gamma_{4}(k x,-k y ; k x,-k y)=i g \frac{\Delta^{2}}{\varepsilon^{2}(k)}, \\
& \Gamma_{4}(k x,-k y ; k y,-k x)=-i g .
\end{aligned}
$$

As long as we are interested in only the first order result for $\mathcal{S}$, we can set $Z=1$ and directly use these expressions to get the following results for the leading low $k$ behaviour of $S_{1}, S_{2}$, and $S_{3}$ correct to first order in $g$ :

$$
\begin{aligned}
& S_{1}(k)=\frac{i g}{2 v_{F}}\left(\frac{k v_{F}}{\Delta}\right), \\
& S_{2}(k)=1+\frac{i g}{2 v_{F}}\left(\frac{\Delta}{k v_{F}}\right), \\
& S_{3}(k)=-\frac{i g}{2 v_{F}}\left(\frac{\Delta}{k v_{F}}\right) .
\end{aligned}
$$

We immediately see that the perturbative expansion cannot be trusted in the low-momentum limit because of the infrared divergences present in the expressions for $S_{2}$ and $S_{3}$. The 
structure of this first order result is seen to be qualitatively similar to the $\mathcal{O}(1 / N)$ result for the two-particle $\mathcal{S}$-matrix of the $O(N) \sigma$-model [10]. In the latter case, we know that the exact value of the $\mathcal{S}$-matrix is perfectly well-behaved in the $k \rightarrow 0$ limit and is in fact given by the super-universal expression (1.2). To obtain the correct result in this limit for our problem, we need to identify the leading infrared divergences at each order in $g$ and perform a resummation. Now, we do not expect any infrared divergences in the perturbation expansion of $Z$ and as a result the prefactor of $(\sqrt{Z})^{4}$ in the expression for $\mathcal{M}$ does not contribute at all to the terms that need to be resummed; we will forget about this factor from now on.

Let us now try and identify the leading infrared divergent diagrams at each order in perturbation theory. First of all, it is clear, purely from frequency and momentum conservation at each vertex, that no diagrams involving pair creation or annihilation can provide the leading divergence at any order. Moreover, only internal loops in which both propogators involved point in the same direction give a nonzero result on doing the integral over the frequency running through the loop. A little thought should convince the reader that these two constraints allow us to conclude that the ladder series shown in Fig 8 give the leading infrared divergent terms in $S_{2}$ and $S_{3}$ to all orders in $g$. Turning our attention to $S_{1}$, we see immediately that Fermi statistics guarantees that each vertex in the analogous ladder series for $S_{1}$ has enough factors of momentum associated with it to rule out any infrared divergence appearing in $S_{1}$. Our task is thus reduced to evaluating the two series shown in Fig 8. To do this, we note that as far as the coefficient of the divergent piece is concerned, we can ignore the momentum dependence of each vertex and simply replace it with a factor of $-i g$. Each crossing of the fermion propogators gives a factor of -1 and each loop integral gives $\Delta / 2 k v_{F}^{2}$. Putting all this together and summing the resultant geometric series, we obtain the following non-perturbative results for the low momentum behaviour of $S_{2}$ and $S_{3}$ :

$$
\begin{aligned}
& S_{2}(k)=\frac{2 i k v_{F}^{2}}{g \Delta+2 i k v_{F}^{2}}, \\
& S_{3}(k)=\frac{g \Delta}{g \Delta+2 i k v_{F}^{2}} .
\end{aligned}
$$

An interesting feature of these results is the pole in the upper-half $k$ plane at $k=i \Delta g / 2 v_{F}^{2}$ which seems to suggest the presence of a bound state. However, this region of $k$ space is definitely beyond the domain of validity of (2.40) and it is not clear what significance, if any, to ascribe to this curious fact.

Turning to firmer ground, we see that the foregoing implies that the low-momentum limit of the two particle $\mathcal{S}$-matrix is perfectly well-defined and is in fact given by

$$
\mathcal{S}_{\mu_{1}^{\prime}, \mu_{2}^{\prime}}^{\mu_{1} \mu_{2}}\left(k_{1}, k_{2} ; k_{1}^{\prime}, k_{2}^{\prime}\right)=\delta_{\mu_{1} \mu_{2}^{\prime}} \delta_{\mu_{2} \mu_{1}^{\prime}} 2 \pi \delta\left(k_{1}-k_{1}^{\prime}\right) 2 \pi \delta\left(k_{2}-k_{2}^{\prime}\right) .
$$

Note that apart from an overall factor of minus one, this is exactly the super-universal form (1.2). The relative sign is simply a consequence of fermi statistics and our choice of phase for the final state of the scattering process. In any case, we will see that when we use the superuniversal form of the $\mathcal{S}$-matrix for discussing spin transport, the overall phase is immaterial. On the other hand, the overall factor of -1 in the superuniversal form (1.2) will be crucial when we work out the correlators of the staggered component of the magnetization density. This may seem worrisome at first sight. However, as we do not have any local representation 
of the staggered component of the spin density in terms of the Majorana fermion operators, there is no contradiction at all. In fact, the semiclassical techniques used in Section IIIA may also be applied to the problem of calculating the finite temperature Green function of the fermions; this would correspond to calculating the finite temperature correlators of some highly non-local string operators of the original spin system. However, as it is difficult to see how these may be accessible at all to any experimental probes, we do not pursue this line of thought any further.

Thus, we see that the low-momentum behaviour of the $\mathcal{S}$-matrix in this fermionic representation of the weakly-coupled ladder is consistent with the super-universal form (11.2), although this behaviour is definitely not accessible to perturbation theory. This leads us to believe that similar infrared divergences would invalidate any perturbative calculation of dynamical properties at finite temperature (when there will be a dilute gas of thermally excited particles present) that uses this representation. In particular, this appears to indicate that the results of Ref [26] for the NMR relaxation rate $1 / T_{1}$ are incorrect at low $T>0$.

\section{DYNAMICS AND TRANSPORT FOR $0<T \ll \Delta$}

The results of this section are expected to apply to all gapped one-dimensional antiferromagnets with massive spin-one quasiparticles. We will develop, what we believe is an exact semiclassical theory of dynamics and transport for $T \ll \Delta$. We will consider fluctuations near $q=Q$ in Section IIIA, and near $q=0$ in Section $\amalg$ II B.

\section{A. Thermal broadening of the single-particle peak in $S(q, \omega)$}

In this section, we present calculations leading up to our results for the thermal broadening of the single particle peak in the dynamics structure factor.

The inelastic neutron scattering cross-section provides a direct measure [1] of the dynamical structure factor $S(q, \omega)$ which is defined as

$$
S(q, \omega)=\frac{1}{2 \pi} \int d t e^{i \omega t}\left\langle\hat{s}_{\alpha}(q, t) \hat{s}_{\alpha}(-q, 0)\right\rangle ;
$$

where $\hat{s}_{\alpha}(q, t)$ is the Heisenberg representation operator corresponding to the component of the spin density at wavevector $q$, the expectation values are with respect to the usual equilibrium density matrix and summation over the repeated index $\alpha$ is implied (note that we are assuming rotational invariance in spin space and working at $H=0$ ). We are interested in the structure factor for $q$ close to $\pi / a$. In this case we have

$$
S(q, \omega) \propto \frac{1}{2 \pi} \int d t e^{i \omega t}\left\langle\hat{n}_{\alpha}(k, t) \hat{n}_{\alpha}(-k, 0)\right\rangle ;
$$

where $k=q-\pi / a$. To get a feel for what (3.2) looks like at $T=0$, let us consider a particular lattice regularization of the $\sigma$-model, defined by the quantum rotor Hamiltonian

$$
\mathcal{H}=\frac{g}{2} \sum_{i} \hat{\mathbf{L}}_{i}^{2}-\frac{1}{g} \sum_{i} \hat{\mathbf{n}}_{i} \cdot \hat{\mathbf{n}}_{i+1}
$$


where $\hat{\mathbf{L}}_{i}$ is the angular momentum operator of the rotor at site $i, \hat{\mathbf{n}}_{i}$ is the unit vector that denotes the position of the rotor at site $i$ and we have temporarily set $c=a=1$. It is not hard to analyze the properties of this model in a large $g$, strong coupling expansion; moreover this is expected to be qualitatively correct for all $g$ in one dimension [47. To lowest order, we can easily see that the ground state would just be a product state with each site being in an eigenstate of $\hat{\mathbf{L}}$ with zero eigenvalue. The lowest excited states would be a degenerate manifold corresponding to promoting any one site to the $L=1$ state and thereby creating a 'particle' at that site. To first order in $1 / g$, a hopping term would be generated in the effective Hamiltonian for the single-particle sector, resulting in a band of one particle excitations. To this order, $\hat{\mathbf{n}}$ is just a sum of creation and annihilation operators for the stable particle-like excitation of the system. At higher orders in $1 / g$, $\hat{\mathbf{n}}$ acting on the vacuum will also produce multiparticle states, but there will always be some single particle component. Reverting back to our continuum theory, we see that (3.2) evaluated at $T=0$ would have a contribution $\sim \delta(\omega-\varepsilon(k))$ associated with the stable particle. The next contribution is actually a continuum above the 3-particle threshold [48]. Following [23], we shall now focus exclusively on how this one-particle peak broadens as $T$ becomes non-zero. Let us define

$$
C(x, t)=\frac{1}{3}\left\langle\hat{n}_{\alpha}(x, t) \hat{n}_{\alpha}(0,0)\right\rangle,
$$

where the repeated index $\alpha$ is summed over. Let $K(x, t)$ denote $C(x, t)$ evaluated at $T=0$ keeping only the single particle contributions. We have

$$
K(x, t)=\int \frac{d p}{2 \pi} D(p) e^{i p x-i \varepsilon(p) t} .
$$

Here $D(p)$ is a 'form factor'. For our Lorentz invariant continuum model,

$$
D(p)=\frac{\mathcal{A} c}{2 \varepsilon(p)}
$$

where $\mathcal{A}$ is a non-universal quasiparticle residue. This gives $K(x, t)=\mathcal{A} K_{0}\left(\Delta\left(x^{2}-\right.\right.$ $\left.\left.c^{2} t^{2}\right)^{1 / 2} / c\right) /(2 \pi)$, with $K_{0}$ the modified Bessel function.

Now let us evaluate $C(x, t)$ for non-zero temperatures using the semiclassical method of [23]. First, it is convenient to switch to operators $n_{+1}(x), n_{-1}(x)$ and $n_{0}(x)$, defined as

$$
n_{+1}=n_{-1}^{\dagger}=n_{x}-i n_{y},
$$

and

$$
n_{0}=n_{z} .
$$

$n_{+1}$ is a sum of a creation operator for particles with $z$-component of spin $m$ equal to +1 and an annihilation operator for particles with $m$ equal to -1 . $n_{0}$ is a sum of creation and annihilation operators for particles with $m$ equal to 0 . In the absence of an external field, we may write

$$
C(x, t)=\left\langle\hat{n}_{0}(x, t) \hat{n}_{0}(0,0)\right\rangle .
$$


We represent (3.6) as a 'double time' path integral, with the $e^{-i \hat{\mathcal{H}} t}$ factor coming from the Heisenberg operator generating paths that move forward in time, and the $e^{+i \hat{\mathcal{H}} t}$ producing paths that move backward in time. We begin with an initial state which is populated by thermally excited particles, the density of particles being $\sim e^{-\Delta / T}$ and their mean spacing being much larger than the thermal de-Broglie wavelength $\sim c /(\Delta T)^{-1 / 2}$. As argued in [22,23], this means that the particles can be treated semiclassically. In this semiclassical limit the dominant contribution to the Feynman sum comes about when the paths going backward in time are exactly the time-reversed counterparts of those going forward and all particles follow their classical trajectories between collisions [22,23]. Whenever two particles collide, energy and momentum conservation is sufficient to determine the final momenta. However, one cannot entirely ignore quantum effects of the collisions. The spins of the particles after the collision as well as the phase picked up by the wavefunction of the system as a result of the collision is determined by the quantum mechanical scattering matrix $(\mathcal{S})$. For $T \ll \Delta$, the particles all move very slowly and we need only the super-universal lowmomentum limit (1.2).

All this leads to the following description of $C(x, t)$ in this asymptotic limit [22]: At time $t=0$ we begin with an initial state populated equally (for $H=0$ ) with three species (corresponding to the three values of spin projection $m$ ) of particles each uniformly distributed in space with density $\rho / 3$, where the total density $\rho$ is given as

$$
\rho=3 \int \frac{d p}{2 \pi} e^{-\left(\Delta+c^{2} p^{2} / 2 \Delta\right) / T}=3 \sqrt{\frac{T \Delta}{2 \pi c^{2}}} e^{-\Delta / T} .
$$

The velocities are distributed according to the classical Maxwell-Boltzmann distribution function

$$
\mathcal{P}(v)=\sqrt{\frac{\Delta}{2 \pi c^{2} T}} e^{-\Delta v^{2} / 2 c^{2} T} .
$$

Each particle in the initial state is assigned one of the three values of $m$ with equal probability (assuming $H=0$ ). The operator $n_{0}(0)$ acting on this initial state creates at time $t=0$ one extra particle at $x=0$ with spin value equal to 0 (the annihilation part of $n_{0}$ gives a contribution which is exponentially suppressed and is ignored here). These particles follow their classical trajectories forward in time. At every collision, we pick up a factor of -1 from the $\mathcal{S}$-matrix. At time $t$, a particle with spin projection of zero is annihilated at $x$ by $n_{0}(x)$. The resulting state is then propogated backward in time to $t=0$ and its overlap with the initial state calculated. $C(x, t)$ is then given by the average of this overlap over the ensemble specified earlier.

A typical example of a space-time configuration of trajectories that leads to a non-zero value for this overlap is shown in Fig 9. All trajectories in the figure except the dotted line denote space-time paths that are traversed both forward and backward in time. The dotted line is traversed only forward in time as the particle travelling on it is destroyed at time $t$ by $n_{0}(x)$. A little thought convinces one that this overlap is non-zero only when all particles colliding with a particle travelling on the dotted trajectory have the same spin $m$ (equal to zero) as it does. Moreover, when this condition is satisfied, the value of the overlap is just $(-1)^{n_{l}} K(x, t)$ where $n_{l}$ is the number of collisions that the dotted trajectory suffers. The 
factor of $(-1)^{n_{l}}$ comes from the scattering matrix at each collision between a particle on the dotted trajectory and other particles. All other collisions occur in pairs (the second member of the pair coming from the evolution backward in time) and thus do not contribute any phase factor. The factor of $K(x, t)$ is just the relativistic amplitude for the propogation of a single particle from $x=0$ at $t=0$ to position $x$ at time $t$.

All this implies that we can write

$$
C(x, t)=R(x, t) K(x, t)
$$

which defines the 'relaxation function' $R$. For the case where the particle has only one allowed value of its spin label, $m$, it is possible to compute $R(x, t)$ analytically [23], and the resulting expression (3.7) then agrees precisely with a computation using very sophisticated quantum inverse scattering method [27]: this agreement gives us confidence that the physical approach developed here is asymptotically exact at low temperatures.

Let us now turn to the calculation of $R$ for the case of interest here. We begin by writing a formal expression for $R$ based on the foregoing semiclassical description. Let $\left\{x_{k}(0)\right\}$ be the positions of the thermally excited particles at time $t=0$. Let $\left\{v_{k}\right\}$ be their initial velocities. Here $k$ is an index running from 1 to $N$, the total number of particles present in the initial state in a system of size $L$. We label the initial positions with the convention that $x_{k}(0)<x_{l}(0)$ for $k<l$. Let $X_{k}(t) \equiv x_{k}(0)+v_{k} t$ denote the $k^{t h}$ space-time trajectory (note that this is quite distinct from the position of the $k^{t h}$ particle at time $t$ ). Let $m_{k}(t)$ denote the spin projection value of the particle travelling along the $k^{\text {th }}$ trajectory at time $t$. The spin projections are randomly assigned to each particle at time $t=0$ as described earlier and $m_{k}(t)$ at later times depends on which particle is travelling on the $k^{\text {th }}$ trajectory at any given time. We have the following expression for $R$ :

$$
R(x, t)=\left\langle\prod_{k} \mathcal{F}_{k}\right\rangle,
$$

with

$$
\mathcal{F}_{k}=1-\left(1+\delta_{m_{k}\left(\tau_{k}\right), 1}\right) \tilde{\Theta}_{k}
$$

where

$$
\tilde{\Theta}_{k}=\theta\left(x-X_{k}(t)\right) \theta\left(x_{k}(0)\right)+\theta\left(X_{k}(t)-x\right) \theta\left(-x_{k}(0)\right),
$$

and

$$
\tau_{k}=x_{k}(0) t /\left(x-v_{k} t\right) .
$$

The angular brackets in (3.8) denote averaging over the ensemble of initial conditions specified earlier.

Unfortunately, it does not seem possible to do the ensemble average analytically. Using methods of Refs [49.50, it is possible to develop a 'cumulant' expansion for the logarithm of $R$ [51]. This expansion, however, is essentially a short time expansion which is not uniformly convergent, and thus not very useful for our purposes as we eventually need to 
Fourier transform $C(x, t)$. It is also possible to develop a 'mean-field' approximation to this classical model that ignores the complicated correlations between the $m_{k}(t)$ at different times (see Appendix D). This proves to be reasonably accurate at least for the $R(0, t)$, though the high-accuracy numerics we describe next show clear deviations from the meanfield results. So, although we have an asymptotically exact formulation for the non-zero temperature $C(x, t)$ at distances much larger than the thermal de-Broglie wavelength and times much larger than $T^{-1}$, we need to numerically determine the relaxation function $R$ to actually calculate anything accurately. This is what we turn to next.

An important property of $R(x, t)$, which follows directly from (B.8) is that it can be written in a scaling form as $R(x, t)=\tilde{R}(\tilde{x}, \tilde{t})$ with $\tilde{x}=x / L_{x}$ and $\tilde{t}=t / L_{t}$ where

$$
L_{x}=\frac{1}{\rho}
$$

and

$$
L_{t}=\frac{1}{\rho}\left(\frac{\Delta}{2 c^{2} T}\right)^{1 / 2} .
$$

Thus it is most convenient for the numerics to measure length in units of $L_{x}$ and time in units of $L_{t}$ and directly calculate $\tilde{R}$. We start with a system size of $L=400$ (in units of $L_{x}$ ) and impose periodic boundary conditions. The density in these units is unity and so the initial state is populated by 400 particles with their initial positions drawn from a uniform ensemble. This system size is large enough that finite-size effects are negligible for our purposes. Each particle is assigned a velocity from the classical thermal ensemble. In these new units this implies that we choose velocities from the distribution

$$
\tilde{\mathcal{P}}(\tilde{v})=\frac{1}{\sqrt{\pi}} e^{-\tilde{v}^{2}} .
$$

An important advantage of our method is that we do the average over the spin values analytically. To do this, we note that it is possible to reformulate the calculation of $\tilde{R}$ by writing

$$
\tilde{R}=\langle T(\mathcal{C})\rangle
$$

where $\mathcal{C}$ denotes a given space time configuration of trajectories, the angular brackets denote averages only over the initial positions and velocities that define this configuration, and $T(\mathcal{C})$ is defined as

$$
T(\mathcal{C})=(-1)^{n_{h}}\left(\frac{1}{3}\right)^{n_{p}}
$$

Here, $n_{h}$ is the total number of collisions involving a particle travelling on the dotted trajectory of Fig $\mathbf{9}$ and $n_{p}$ is the number of different thermally excited particles that have had collisions with a particle travelling on the dotted trajectory. Now, $T(\mathcal{C})=0$ for all configurations $\mathcal{C}$ in which the presence of the extra particle (that starts out on the dotted trajectory) affects the evolution of the various spin values $m_{k}(t)$. So we might as well forget 
about the particle travelling on the dotted trajectory and consider an auxillary spacetime diagram that now involves only the thermally excited particles. We now agree to ignore the spin label on the dotted line of Fig 9; the dotted line now does not denote the trajectory of any particle. In terms of this picture we can define $n_{h}$ as the number of times any solid line crosses the dotted line, and $n_{p}$ as the number of different thermally excited particles that cross the dotted line.

With this new formulation in hand, calculating $T(\mathcal{C})$ reduces to some simple bookkeeping that keeps track of these two integers for a given configuration $\mathcal{C}$. We implement the ensemble average by averaging over $4 \times 10^{6}$ configurations drawn from the appropriate distribution. The combined absolute error (statistical and finite-size) in $\tilde{R}(\tilde{x}, \tilde{t})$ for values of $\tilde{x}, \tilde{t}$ of interest to us is estimated to be less than about $5 \times 10^{-4}$.

With $\tilde{R}$ available, it is a simple matter to numerically Fourier transform the resulting $C(x, t)$ and obtain the dynamic structure factor $S(q, \omega)$. Details of the numerical procedure used are relegated to Appendix B. Here we only comment on some conceptual issues involved and discuss our results.

There is an important subtlety associated with doing the Fourier transform that needs to be first addressed. As discussed in Ref [23], the semiclassical result for $C(x, t)$ is valid as long as both $x$ and $t$ are not very small; the results break down when $x \sim \lambda_{T}$ and $t \sim 1 / T$ ( $\lambda_{T}$ being the thermal de-Broglie wavelength). However, the Fourier transform of $C$ (at wavevector $k=q-\pi / a)$ is an asymptotically valid approximation to $S(q, \omega)$ only for $\omega$ close to $\varepsilon(k)$. The reason for this can be understood by noting that the long-time asymptotics of our form for $C(x, t)$ have an oscillatory character with oscillations on the scale of $\Delta^{-1}$. Put another way, it is the spectral weight in the one-particle peak that plays a dominant role in determining the long-time, large-distance asymptotics of $C(x, t)$ and so we can learn only about this feature in the spectral weight by Fourier transforming our form for $C$.

With this caveat in mind, we have

$$
S(q, \omega)=\frac{1}{2 \pi} \iint d t d x e^{i \omega t-i k x} K(x, t) R(x, t) .
$$

where $k=q-\pi / a$. We have not attempted to exhaustively map out $S(q, \omega)$, although it would be quite straightforward to get more extensive numerical results should they be of interest in some experimental context. Below we confine ourselves to discussing our results for $S(q, \omega)$ for a couple of sample values of $q$. Fig 10 shows scans in frequency across the quasiparticle peak in $S(q, \omega)$ for $q=Q$ at four different values of temperature. It is interesting to note that when rescaled by $L_{t}$ and plotted against a rescaled frequency variable $\delta \tilde{\omega}=(\omega-\Delta) L_{t}$, the three curves for $\Delta / T=3,4$, and 5 seem to collapse on top of one another within our numerical errors (which are conservatively estimated to be a few percent at the most). In Fig 11, we show a scan in wavevector across the same peak for $\omega=\Delta$, again at the same four values of temperature. The curves at the lower temperatures again show scaling collapse; when rescaled by $L_{t}$ and plotted against the rescaled variable $\hat{k}=k c \sqrt{L_{t} / \Delta}$, they seem to all fall on top of one another. Moreover, the scaling curve in Fig 11, when plotted as a function of the independent variable $-\hat{k}^{2} / 2$ coincides within our numerical error with the scaling function of Fig 10 for $\delta \tilde{\omega}<0$; this is displayed in Fig 12. While we do not have any reason to expect that this scaling is generally true, all 
three observations may be put together in terms of a scaling form that is valid locally in the neighbourhood of the quasiparticle peak for $q=Q$; more formally we write

$$
S(q, \omega)=\frac{\mathcal{A} c L_{t}}{\pi^{2} \Delta} \Phi\left(\frac{\omega-\varepsilon(k)}{L_{t}^{-1}}\right) .
$$

We also investigated $S(q, \omega)$ in the vicinity of the quasiparticle peak corresponding to $q=Q+\Delta / c$; for this to be meaningful, we of course need $\Delta / c$ to be much less than the microscopic scale $\sim a^{-1}$ beyond which our continuum theory does not work. We again tried to check if the analogous scaling form,

$$
S(q, \omega)=\frac{\mathcal{A} c L_{t}}{\pi^{2} \Delta} \Phi_{\Delta / c}\left(\frac{\omega-\varepsilon(k)}{L_{t}^{-1}}\right),
$$

is approximately valid. Fig 13 shows scans in frequency across the peak with $k$ held fixed at $\Delta / c$, for $\Delta / T=2,3,4$, and 5 . We see that the curves do not really appear to scale. In Fig 14, we show scans in wavevector, with $\omega$ held fixed equal to $\sqrt{2} \Delta$ for the same values of the ratio $\Delta / T$. We plot the data (rescaled by $L_{t}$ ) against the rescaled variable $\delta \hat{k}=c L_{t}(k-\Delta / c)$ (note the difference in the choice of rescaling of the independent variable from the earlier case). Again, in sharp contrast to the $q=\pi / a$ peak, we see that the curves do not show any signs of scaling; our local scaling form is not a very good way of organizing the data in this case.

These scaling properties are best understood as follows: Imagine developing $R(x, t)$ in an expansion about $x=0$ for constant $t$ and then calculating the Fourier integral in (3.9). The zeroth order term clearly gives us a result for $S(q, \omega)$ which is compatible with the scaling form we have postulated for asymptotically low temperatures. However, before we can trust this result, we need to check that the corrections to the leading behaviour go to zero in the limit $T \rightarrow 0$. This is where the difference between the two peaks we looked at becomes apparent. It is easy to see that this is true only for values of $q$ such that $c^{2}|k| L_{t} / \Delta L_{x} \rightarrow 0$ as $T \rightarrow 0$ and this explains why the scaling form (3.11) does not work. Now consider the peak at $q=Q$ : The zeroth order scaling result has most of its weight in the region $|k| \leq \sqrt{\Delta} / c \sqrt{L_{t}}$. For $|k| \sim \sqrt{\Delta} / c \sqrt{L_{t}}$, the corrections to this leading result do indeed go to zero and this establishes the scaling form (3.10). An interesting feature of this result is that the scaling function $\Phi$ is completely determined by the $x=0$ part $R(0, t)$ of the relaxation function. More precisely, we have

$$
\Phi(z)=\frac{\pi}{4} \int_{-\infty}^{\infty} d s e^{i z s} \tilde{R}(0, s)
$$

A useful check on all of our calculations is thus to compare the scaling function obtained in Fig 10 and Fig 12 with (3.12) evaluated numerically (it is possible to do this to a high accuracy; details may be found in Appendix B). The results of such a comparison are shown in Fig 15 and the agreement is seen to be quite good. While the numerical results for $R(0, t)$ show a clear deviation from the simple exponential decay predicted by the 'mean-field' theory referred to earlier, we do find that the corresponding simple Lorentzian form for the Fourier transform: $\Phi(z)=\pi \alpha / 2\left(\alpha^{2}+z^{2}\right)$ (with $\alpha \approx 0.71$ ) provides an excellent approximation to 
the line-shape (the 'mean-field' theory, however, gives a value of $4 / 3 \sqrt{\pi} \approx 0.7523$ for $\alpha$ - see Appendix D).

We thus have results for the thermally broadened quasiparticle peak in $S(q, \omega)$; the accuracy of these in the asymptotic regime $(T \ll \Delta)$ is limited only by the computer time spent in numerically evaluating the relaxation function and doing the Fourier transform. These results, especially the scaling properties in the vicinity of the peak corresponding to $q=Q$, should be of relevance to neutron scattering experiments on gapped one-dimensional Heisenberg antiferromagnets performed at temperatures $T \ll \Delta$ and it is hoped that this study provides a useful paradigm for organizing the experimental results.

\section{B. Low temperature spin diffusion probed by $1 / T_{1}$}

In this section, we shall present a detailed comparison of our results [22 for the field $(H)$ and temperature $(T)$ dependence of the NMR relaxation rate $1 / T_{1}$ (in the regime $T, H \ll \Delta$ ) with the experimental data of Ref [30] on the NMR relaxation rate in the compound $\mathrm{AgVP}_{2} \mathrm{~S}_{6}$ which is thought to be a $S=1$ one-dimensional antiferromagnet with a large gap $\Delta \approx 300 \mathrm{~K}$ and single-ion anisotropy energy of about $4.5 \mathrm{~K}$ [30]. We will ignore this anisotropy for the most part in our theoretical analysis (although we are forced to phenomenologically introduce spin-dissipation into our theorerical results in order to fit the data of Ref [30] at low temperatures, we do not have any theory that gives the detailed temperature dependence of this spin dissipation rate starting from the anisotropic coupling term in the Hamiltonian).

For completeness, let us begin with a detailed review of the calculations leading up to our expression for $1 / T_{1}$. The NMR relaxation rate is given in general by an expression of the form

$$
\frac{1}{T_{1}}=\sum_{\alpha=x, y} \sum_{\beta, \gamma=x, y, z} \int \frac{d q}{2 \pi} A_{\alpha \beta}(q) A_{\alpha \gamma}(-q) S_{\beta \gamma}\left(q, \omega_{N}\right)
$$

where $S_{\beta \gamma}(q, \omega)$ is the Fourier transform of the spin-spin correlation function (the subscripts refer to the $O(3)$ indices of the spin operators), $\omega_{N}=\gamma_{N} H$ is the nuclear Larmor frequency $\left(\gamma_{N}\right.$ is the nuclear gyromagnetic ratio), the field $H$ points in the $z$ direction and $A_{\alpha \beta}$ are the hyperfine coupling constants. The $q$ integral in (3.13) is dominated by values of $q$ near 0 [31 and we can thus work out the field and temperature dependence of $1 / T_{1}$ knowing the $T>0$ correlators of the conserved magnetization density of the $O(3)$ NL $\sigma \mathrm{M}$ field theory. This is what we turn to next.

We define the correlation functions

$$
\begin{aligned}
C_{u, z z}(x, t) & =\left\langle\hat{L}_{z}(x, t) \hat{L}_{z}(0,0)\right\rangle-\langle\hat{L}\rangle^{2} \\
C_{u,-+}(x, t) & =\left\langle\hat{L}_{-}(x, t) \hat{L}_{+}(0,0)\right\rangle ;
\end{aligned}
$$

here the angular brackets denote averaging over the usual equilibrium density matrix, $\hat{L}_{z}(x, t)$ is the Heisenberg representation operator corresponding to the $z$ component of the magnetization density, and $\hat{L}_{ \pm}$are operators corresponding to the circularly polarized components 
of the magnetization density defined as $\hat{L}_{ \pm} \equiv \hat{L}_{x} \pm i \hat{L}_{y}$. As argued in Refs [22,23], these correlation functions in the asymptotic regime may be evaluated by writing down a double-time path integral representation for them and evaluating it semiclassically.

This leads to the following prescription [22] for $C_{u, z z}(x, t)$ : At time $t=0$ we begin with an initial state populated with three species (corresponding to the three values of spin projection $m$ ) of particles each uniformly distributed in space with densities given respectively by

$$
\rho_{m}=\int \frac{d p}{2 \pi} e^{-\left(\Delta-m H+c^{2} p^{2} / 2 \Delta\right) / T}=\sqrt{\frac{T \Delta}{2 \pi c^{2}}} e^{-(\Delta-m H) / T},
$$

and with velocities distributed according to the classical Maxwell-Boltzmann distribution function

$$
\mathcal{P}(v)=\sqrt{\frac{\Delta}{2 \pi c^{2} T}} e^{-\Delta v^{2} / 2 c^{2} T} .
$$

Each particle in the initial state is assigned one of the three values of $m$ with probability $f_{m}=e^{m H / T} /(1+2 \cosh (H / T))$. The operator $\hat{L}_{z}(0)$ merely keeps track of the local value of the $z$ component of the spin. Acting on the initial state, it measures the $z$ component of the magnetization density in the initial state at position $x=0$. These particles then follow their classical trajectories forward in time. At every collision, the particles retain their spin labels. In addition, the state picks up a factor of -1 from the $\mathcal{S}$-matrix at each collision. At time $t$, the operator $\hat{L}_{z}(x)$ measures the value of the $z$ component of magnetization density at position $x$. The state is then propogated backward in time to $t=0$ and its overlap with the initial state calculated. $C_{u, z z}(x, t)$ is then given by the average of this overlap over the ensemble specified earlier. As all collisions have a time-reversed counterpart, the phase of the scattering matrix does not matter here and the overlap we are interested in equals the two-point correlation function of the classical observable

$$
\varrho_{z}(x, t)=\sum_{k} m_{k} \delta\left(x-x_{k}(t)\right)
$$

where we are labelling particles consecutively from left to right with an index $k, x_{k}(t)$ denotes the position of the $k^{t h}$ particle at time $t$, and $m_{k}$ is the $z$ component of the spin of the $k^{t h}$ particle. This correlation function is calculated using the ensemble of initial conditions outlined above. The dynamics governing the time evolution of the $x_{k}$ is just that of particles moving ballistically except for elastic collisions in which they retain their spin values.

Thus we can write

$$
C_{u, z z}(x, t)=\sum_{k, l}\left\langle m_{k} \delta\left(x-x_{k}(t)\right) m_{l} \delta\left(x_{l}(0)\right)\right\rangle-\left\langle\varrho_{z}\right\rangle^{2}
$$

here the angular brackets refer to averaging over the ensemble of spin labels $m_{k}$, initial velocities $v_{k}(0)$, and initial positions $x_{k}(0)$ specified earlier. Now as the spin-projections $m_{k}$ are not correlated with the initial positions or velocities, the averages factorize. The correlators of the $m_{k}$ are easily evaluated as: 


$$
\left\langle m_{k} m_{l}\right\rangle=A_{1}+A_{2} \delta_{k l}
$$

where $A_{1}=\left(f_{1}-f_{-1}\right)^{2}$ and $A_{2}=f_{1}+f_{-1}-\left(f_{1}-f_{-1}\right)^{2}$ are simple, dimensionless, known functions of $H / T$ only. Using (3.17) we have

$$
\begin{gathered}
C_{u, z z}(x, t)=A_{1}\left(\langle\rho(x, t) \rho(0,0)\rangle-\rho^{2}\right) \\
+A_{2} \sum_{k}\left\langle\delta\left(x-x_{k}(t)\right) \delta\left(x_{k}(0)\right)\right\rangle
\end{gathered}
$$

where $\rho(x, t)=\sum_{k} \delta\left(x-x_{k}(t)\right)$ is the spacetime dependent total density, all averages are now with respect to initial positions and velocities, and $\rho \equiv\langle\rho(x, t)\rangle=\sum_{m} \rho_{m}$. The twopoint correlators of $\rho(x, t)$ are also easy to evaluate: if the spin labels are neglected, the collisions have no effect and correlators of the total denstiy can be obtained by considering an ideal gas of point particles. The second correlator in (3.18), multiplying $A_{2}$, is more difficult: it involves the self two-point correlation of a given particle $k$, which follows a complicated trajectory. Fortunately, precisely this correlator was considered three decades ago by Jepsen [49] and a little later by others [50]; they showed that, at sufficiently long times, this correlator has a Brownian motion form. In Appendix C, we give a self- contained summary of Jepsen's calculation. Here we just write down the final results 22 for the correlation function:

$$
C_{u, z z}(x, t)=\rho^{2}\left[A_{1} F_{1}\left(\frac{|x|}{L_{x}}, \frac{|t|}{L_{t}}\right)+A_{2} F_{2}\left(\frac{|x|}{L_{x}}, \frac{|t|}{L_{t}}\right)\right]
$$

where $\rho^{2} F_{1}$ is the connected density correlator of a classical ideal gas in $d=1$,

$$
F_{1}(\tilde{x}, \tilde{t})=e^{-\tilde{x}^{2} / \tilde{t}^{2}} / \tilde{t} \sqrt{\pi}
$$

and $\rho^{2} F_{2}$ is the correlator of a given labeled particle 49, 50,

$$
\begin{aligned}
F_{2}(\tilde{x}, \tilde{t})= & {\left[\left(2 G_{1}(u) G_{1}(-u)+F_{1}(\tilde{x}, \tilde{t})\right)\right.} \\
& \times I_{0}\left(2 \tilde{t} \sqrt{G_{2}(u) G_{2}(-u)}\right) \\
& +\frac{G_{1}^{2}(u) G_{2}(-u)+G_{1}^{2}(-u) G_{2}(u)}{\sqrt{G_{2}(u) G_{2}(-u)}} \\
\times & \left.I_{1}\left(2 \tilde{t} \sqrt{G_{2}(u) G_{2}(-u)}\right)\right] e^{-\left(G_{2}(u)+G_{2}(-u)\right) \tilde{t}}
\end{aligned}
$$

with $u \equiv \tilde{x} / \tilde{t}, G_{1}(u)=\operatorname{erfc}(u) / 2$, and $G_{2}(u)=e^{-u^{2}} /(2 \sqrt{\pi})-u G_{1}(u)$. For $|\bar{t}| \ll|\bar{x}| \ll 1$, the function $F_{2}$ has the ballistic form $F_{2}(\bar{x}, \bar{t}) \approx F_{1}(\bar{x}, \bar{t})$, while for $|\bar{t}| \gg 1,|x|$ it crosses over to the diffusive form

$$
F_{2}(\bar{x}, \bar{t}) \approx \frac{e^{-\sqrt{\pi} \bar{x}^{2} / 2 \bar{t}}}{\left(4 \pi \bar{t}^{2}\right)^{1 / 4}} \quad \text { for large } \bar{t}
$$


In the original dimensionful units, (3.22) implies a spin diffusion constant, $D_{s}$, given exactly by

$$
D_{s}=\frac{c^{2} e^{\Delta / T}}{\Delta(1+2 \cosh (H / T))} .
$$

This result has been obtained by the solution of a classical model which possesses an infinite number of local conservation laws: in Appendix A, we explicitly show how the existence of these local conservation laws is not incompatible with diffusive spin dynamics. It must be noted that the result (3.23) does not imply that we have rigorously established that the ultimate long-time corelations of the quantum model are also diffusive: the reasons for this and related comments were made earlier in Section 1 below (1.5).

Let us now summarize the calculation of the correlator of the transverse components of the magnetization density. The semiclassical prescription for evaluating $C_{u,-+}(x, t)$ is again quite straightforward: We begin with an initial state chosen from the same ensemble as before. $\hat{L}_{+}(0)$ acting on the initial state gives zero unless there is a particle at $x=0$ with spin label $m=0,-1$, in which case it raises the $m$ value of that particle by 1 and multiplies the state by a factor of $\sqrt{2}$ (coming from the usual properties of raising operators for the spin-one representation of the angular momentum algebra). The resulting state is then propogated forward in time with all the particles moving along their classical trajectories as before. At time $t$, the operator $\hat{L}_{-}(x)$ acting on this state gives zero unless there is a particle at $x$ with spin label $m=0,1$, in which case it lowers the spin value of that particle by 1 and again multiplies the state by a factor of $\sqrt{2}$. This state is then propogated backward in time and its overlap with the initial state calculated. $C_{u,-+}(x, t)$ is given by this overlap averaged over the ensemble of initial conditions. Here, as before, the phase factor of -1 coming from each collision does not matter as each collision has a time reversed counterpart. Also, it is easy to see that in this case the overlap with the initial state is zero unless $\hat{L}_{-}(x)$ lowers the spin of precisely the particle whose spin was raised by $\hat{L}_{+}(0)$. Lastly, we see that there is an overall factor of $e^{+i H t}$ coming from the unitary time evolution as the total spin of the state during its evolution forward in time is greater than the total spin during its evolution backward in time by precisely one. Similar considerations apply to $C_{u,+-}$. Putting all of this together we see that

$$
C_{u, \mp \pm}(x, t)=2 \rho^{2} e^{ \pm i H t} A_{\mp} F_{2}\left(\frac{|x|}{L_{x}}, \frac{|t|}{L_{t}}\right)
$$

where $A_{\mp} \equiv f_{0}+f_{\mp 1}$.

Now, we may express the NMR relaxation rate in terms of the correlation functions of the conserved magnetization density as

$$
\frac{1}{T_{1}}=\sum_{\alpha=x, y} \sum_{\beta, \gamma=x, y, z} A_{\alpha \beta} A_{\alpha \gamma} S_{\beta \gamma}\left(\omega_{N}\right),
$$

where the local dynamic structure factor $S_{\beta \gamma}\left(\omega_{N}\right)$ is defined as

$$
S_{\beta \gamma}\left(\omega_{N}\right)=\int d t e^{i \omega_{N} t} C_{u, \beta \gamma}(0, t)
$$


note that we have neglected the $q$ dependence of the hyperfine couplings and ignored the contribution of the antiferromagnetic spin fluctuations to the integral over $q$ in (3.13). At this point we have to address an important subtlety that arises in calculating the local dynamic structure factor from the autocorrelation function. We are treating the spin dynamics semiclassically to arrive at our expressions for the correlation functions. This gives rise to a characteristic $1 / t$ divergence at short times in the corresponding autocorrelation functions. This is basically a signature of classical ballistic spin transport; at these short time scales collisions play no role. As a result, the integral as written is logarithmically divergent at short times. Our semiclassical expressions for the correlation functions do not make sense for very short times. This is natural as our whole approach has been geared towards calculating these correlations at time scales much larger than $1 / T$ and length scales much larger than the thermal de broglie wavelength; our method fails when both these conditions are simultaneously violated [23]. The semiclassical expressions for $C_{u}(0, t)$ are thus only valid for $t>\epsilon_{t}$ where $\epsilon_{t}$ is a short time cutoff $\sim 1 / T$. Introducing this short time cutoff will give a well- defined result for $S_{\alpha \beta}\left(\omega_{N}\right)$ at the price of introducing an arbitrary scale $\epsilon_{t} \sim 1 / T$; this does not seem very promising as our results for $S_{z z}\left(\omega_{N}\right)\left(S_{ \pm \mp}\left(\omega_{N}\right)\right)$ will depend sensitively upon $\epsilon_{t}$ except for very small fields such that we are in the collision dominated diffusive regime: $\gamma_{N} H \ll 1 / L_{t}\left(H \ll 1 / L_{t}\right)$. Note that the range of $H$ for which the results are insensitive to the cutoff differs for the transverse components of the local dynamic structure factor because of the overall oscillatory factor of $e^{ \pm i H t}$ in the corresponding autocorrelation functions (this factor always dominates as $\gamma_{N} \ll 1$ ). However, we can still use our approach to compute the $S_{\alpha \beta}\left(\omega_{N}\right)$. The point is that, at very short times, the collisions between the thermally excited particles do not matter, and the spin dynamics is ballistic. This means that $S_{\alpha \beta}\left(\omega_{N}\right)$, for high frequencies $\omega_{N}$ (such that $\omega_{N}$ is much larger than the mean collision rate $\left.\sim 1 / L_{t}\right)$, may be calculated exactly by doing a full quantum calculation for a gas of noninteracting spin-one particles 31. Now, we can expand our semiclassical result (obtained by using a cutoff $\epsilon_{t}$ ) for $\omega_{N} \gg 1 / L_{t}$ and match the leading term in this large $H$ expansion with the small $H$ limit (for $S_{z z}\left(S_{ \pm \mp}\right)$ this would be the regime $\gamma_{N} H \ll T(H \ll T)$ ) of the quantum calculation of Ref 31. This, then, will uniquely fix $\epsilon_{t}$ and give us results for the $S_{\alpha \beta}\left(\omega_{N}\right)$ that will work reasonably well even for $H \sim T$ (though strictly speaking they are valid only in the range $\gamma_{N} H \ll T(H \ll T)$ for $\left.S_{z z}\left(S_{ \pm \mp}\right)\right)$.

To see explicitly how this procedure works, consider $S_{z z}\left(\omega_{N}\right)$. It is quite easy to see that the $\omega_{N} \gg 1 / L_{t}$ limit of the semiclassical $S_{z z}\left(\omega_{N}\right)$ is:

$$
\frac{\Delta e^{-\Delta / T}}{\pi c^{2}}\left(e^{H / T}+e^{-H / T}\right) \ln \left(\frac{e^{-\gamma}}{\epsilon_{t} \omega_{N}}\right),
$$

where $\gamma \approx 0.577216$ is Euler's constant. The $\omega_{N} \ll T$ limit of the full quantum calculation reads 31 :

$$
\frac{\Delta e^{-\Delta / T}}{\pi c^{2}}\left(e^{H / T}+e^{-H / T}\right) \ln \left(\frac{4 T e^{-\gamma}}{\omega_{N}}\right) .
$$

Thus we can set $\epsilon_{t}=1 / 4 T$ to match the two logarithms. It is easy to check that the same choice works for the transverse correlators. It is now quite straightforward to do the $t$ integrals and obtain the following results [22] for the local dynamic structure factor: 


$$
\begin{gathered}
S_{z z}\left(\omega_{N}\right)=\frac{\rho}{c} \sqrt{\frac{2 \Delta}{\pi T}}\left[A_{1}\left\{\ln \left(T L_{t}\right)+\Phi_{1}\left(\sqrt{\pi}\left|\omega_{N}\right| L_{t}\right)\right\}\right. \\
\left.+A_{2}\left\{\ln \left(T L_{t}\right)+\Phi_{2}\left(\sqrt{\pi}\left|\omega_{N}\right| L_{t}\right)\right\}\right], \\
S_{\mp \pm}\left(\omega_{N}\right)=\frac{2 \rho A_{\mp}}{c} \sqrt{\frac{2 \Delta}{\pi T}}\left\{\ln \left(T L_{t}\right)+\Phi_{2}\left(\sqrt{\pi}\left|\omega_{N} \pm H\right| L_{t}\right)\right\} .
\end{gathered}
$$

The $\ln \left(T L_{t}\right)$ terms logarithmically violate the purely classical, reduced scaling forms [36], and were fixed using the matching procedure just outlined. The scaling functions $\Phi_{1,2}(\Omega)$ were determined in Ref [22] to be

$$
\begin{aligned}
\Phi_{1}(\Omega)= & \ln \left(\frac{4 \sqrt{\pi} e^{-\gamma}}{\Omega}\right), \\
\Phi_{2}(\Omega)= & \Phi_{1}(\Omega)+\frac{\pi\left[\left(\sqrt{4+\Omega^{2}}+2\right)^{1 / 2}-\sqrt{\Omega}\right]^{2}}{4 \sqrt{\Omega}\left(\sqrt{4+\Omega^{2}}+2\right)^{1 / 2}} \\
& -\ln \frac{\left(1+\Omega^{2} / \Psi^{2}(\Omega)\right)^{1 / 2}(1+\Psi(\Omega))}{2 \Omega} ;
\end{aligned}
$$

where $\gamma=0.57721 \ldots$ is Euler's constant, and $\Psi(\Omega)=\left(\Omega \sqrt{1+\Omega^{2} / 4}-\Omega^{2} / 2\right)^{1 / 2}$. Note that the above expression for $\Phi_{2}(\Omega)$ clearly shows the expected crossover from the large frequency ballistic behavior $\Phi_{2}(\Omega \rightarrow \infty)=\ln (1 / \Omega)$, to the small frequency diffusive form $\Phi_{2}(\Omega \rightarrow 0)=\pi /(2 \sqrt{\Omega})$.

Let us now use all of this to make contact with the experimental results of Ref [30]. For this particular experimental setup, the expression for $1 / T_{1}$ simplifies and to a very good approximation we can write [30]

$$
\frac{1}{T_{1}}=\Gamma_{1} \times S_{x x}\left(\omega_{N}\right)
$$

here the relevant hyperfine coupling constant is known [30 to have the value $\Gamma_{1} \approx(7.5 \times$ $\left.10^{5}\right)^{\circ} K \sec ^{-1}$ (note that we have used units such that $\hbar=k_{B}=1$ in our computation of the correlation functions and thus time is being measured in inverse Kelvins). To begin with, we straightforwardly attempt to fit the field dependent $1 / T_{1}$ with our results. We use the values $\Delta=320 \mathrm{~K}$ and $c=3.32 \Delta$ (we are working in units where the lattice constant $a$ is set to one) extracted from the susceptibility data [52]. In actual fact, we introduce an additional, field-independent background rate $R_{b}$ that we add on to our theoretical result for $1 / T_{1}$. This serves as our fitting parameter; we choose it at each temperature to achieve the best agreement with the results of Ref [30]. We show the resulting fits for $T=320,220$, and $160 \mathrm{~K}$ in Fig 16. We see that the theoretical curves account for the field dependence of $1 / T_{1}$ extremely well in this temperature range (of course the agreement for $T=320 \mathrm{~K}$ should not be taken too seriously as our theory is valid only for temperatures smaller than the gap). In particular, the data seems to clearly exhibit the theoretically predicted $1 / \sqrt{H}$ divergence at low fields which is a characteristic of diffusive spin dynamics. In Fig 17 and Fig 18, we compare the theoretical predictions with the experimental data at $T=120$, 100, 90, 80, 70, and $60 \mathrm{~K}$. At these lower temperatures this divergence seems to get cut 
off below some threshold field and the quality of the fit deteriorates rapidly. This indicates the presence of some spin-dissipation mechanism which becomes significant at these lower temperatures and rounds off the diffusive $1 / \sqrt{\omega}$ divergence in the local dynamic structure factor. Both inter-chain coupling and single-ion anisotropy of the intra-chain coupling are expected to contribute to the spin dissipation rate. However, we do not have any real theory that can work out the effects of these terms in the Hamiltonian on the field and temperature dependence of $1 / T_{1}$.

We can only attempt to phenomenologically introduce some spin dissipation in our theoretical results for the spin correlators. Following [30], we do this by simply introducing an exponential cutoff to the long-time tail of the autocorrelation function; thus we write $C_{u, x x}^{\prime}(0, t)=e^{-\gamma t} C_{u, x x}(0, t)$. It is straightforward, though somewhat tedious to work out the corresponding local dynamic structure factor by doing the Fourier transform and we will spare the reader the details. This now gives us a phenomenological result for $1 / T_{1}$ with an additional tunable parameter $\gamma$. We choose this spin-dissipation rate at each temperature to achieve the best fit with the data. The resulting curves are shown in Fig 19 and Fig 20 for a few representative temperature values. We see that it is possible to fit the data moderately well; discrepencies are however clearly visible and it is not clear how much significance to attach to the sharp increase in $\gamma$ as the temperature is lowered. The quality of our fit seems at first sight to be much worse than the corresponding fit to a purely classical diffusive form employed in Ref [30]. However, it is important to note that the phenomenological model of Ref [30 used the diffusion constant as an additional fitting parameter; we do not have any such freedom. Moreover, both the diffusion constant and the constant background rate extracted from the fit in Ref [30] take on unphysical values below about $100 \mathrm{~K}$ [30. This is because, at these lower temperatures, we are in the ballistic regime of spin-transport for a significant portion of the $H$ axis and the contribution from the 'free-boson logarithms' cannot be neglected. As the crossover to the ballistic regime is already incorporated in our form, the present results for the background rate do not suffer from any such obvious problems (the diffusion constant of course is just given by (3.23) in our approach). In Fig 21 and Fig 22, we plot the corresponding values of the spin-dissipation rate $\gamma$ and the background rate $R_{b}$ as a function of temperature. The spin dissipation rate is seen to increase rapidly as the temperature is decreased. On the other hand, we see that the temperature dependence of $R_{b}$ may be fit approximately by an activated form with activation gap close to $3 \Delta / 2$.

\section{HIGH $T$ REGION $(T \gg \Delta)$ OF THE CONTINUUM $\sigma$ MODEL}

We consider here the possibility that it may be possible to find gapped spin chains which satisfy $\Delta \ll J$, where $J$ is a typical exchange constant. In this case, it becomes possible to access a higher temperature regime where a continuum field theory description is possible in the regime $\Delta \ll T \ll J$. In particular, we expect that the continuum $\sigma$-model to apply in such a regime [35]. It is our hope that such a universal high $T$ regime can be experimentally accessed in $S=2$ spin chains [34]. Moreover, the study of such a high $T$ regime is of importance as matching its results with the $T \ll \Delta$ theory can, in principle, help us estimate the values of $T$ to which the low $T$ results can be applied.

An important property of this regime [35] is that equal-time two-point correlator of $n_{\alpha}$, 
$C(x, 0)$ (Eqn (3.3)) decays at large $x$ with a correlation length

$$
\xi \sim \frac{c}{T} \ln (T / \Delta) .
$$

We will shortly determine the exact values of the prefactor and the argument of the logarithm in (4.1). At distances of order or shorter than this correlation length we may crudely expect that a weak-coupling, spin-wave picture will hold, and excitations will have energy of order or smaller than $c \xi^{-1}$, which is logarithmically smaller than the thermal energy $T$; in other words

$$
\frac{c \xi^{-1}}{T} \sim \frac{1}{\ln (T / \Delta)}<1
$$

So the occupation number of these spin-wave modes will then be

$$
\frac{1}{e^{c \xi^{-1} / T}-1} \approx \frac{T}{c \xi^{-1}}>1
$$

The last occupation number is precisely that appearing in a classical description of thermally excited spin waves, which suggests that a classical wave description should yield an appropriate picture of the dynamics of this high $T$ region. However, notice that classical thermal effects are only logarithmically preferred, and any predictions of a classical dynamical theory will only be correct to leading logarithms.

We begin our analysis by first focussing on the equal-time correlations in this region. We shall use a method originally introduced by Luscher [53]. The main idea of Luscher is to develop an effective action for only the zero Matsubara frequency $\left(\omega_{n}=0\right)$ components of $n_{\alpha}$ after integrating out all the $\omega_{n} \neq 0$ modes (the $\omega_{n}=0$ modes are related to the equal-time correlations via the fluctuations-dissipation theorem and the Kramers-Kronig relations [54]). This is expected to yield the following partition function for a $\tau$-independent field $n_{\alpha}(x)$ :

$$
Z=\int \mathcal{D} n_{\alpha}(x) \delta\left(n_{\alpha}^{2}-1\right) \exp \left(-\frac{(N-1) \xi}{4} \int d x\left(\frac{d n_{\alpha}(x)}{d x}\right)^{2}\right)
$$

We have now generalized to a field $n_{\alpha}$ with $N$ components, and will quote many of our results for general $N$; the physical case if of course $N=3$. The coupling constant in (4.4) is written in a form such that $\xi$ is the exact correlation length: this follows from the easily computable exact correlations of $Z$ by interpreting it as the quantum mechanics of a single quantum rotor. The value of $\xi$ can be computed in a perturbation theory in $g$ on the quantum model (2.1): the $\omega_{n} \neq 0$ modes can be integrated out using a now standard approach [47]

$$
\frac{(N-1) \xi T}{2}=\frac{c}{g}-c^{2}(N-2) \int \frac{d k}{2 \pi} T \sum_{\omega_{n} \neq 0} \frac{1}{c^{2} k^{2}+\omega_{n}^{2}}
$$

The integral on the right-hand-side is not ultraviolet convergent. We evaluate it using the renormalization procedure discussed by Brezin and Zinn-Justin [55]. We introduce a momentum scale $\mu$ at which coupling constants are defined, and generalize (2.1) to a model in $d$ spatial dimensions. We now define the renormalized dimensionless coupling 


$$
g_{R}(\mu)=\mu^{\epsilon} Z_{1} g
$$

where $\epsilon \equiv 1-d$, and the renormalization constant $Z_{1}$ is determined in dimensional regularization to be [55]:

$$
Z_{1}=1-\frac{(N-2)}{2 \pi} \frac{g_{R}(\mu)}{\epsilon}+\ldots
$$

We now need to express (4.5) in terms of $g_{R}$, and evaluate the integral on the right hand side in $d=1-\epsilon$ dimensions. Let us display a few steps of the latter evaluation:

$$
\begin{aligned}
& T \sum_{\omega_{n} \neq 0} \int \frac{d^{1-\epsilon} k}{(2 \pi)^{1-\epsilon}} \frac{1}{c^{2} k^{2}+\omega_{n}^{2}}=\int \frac{d^{1-\epsilon} k}{(2 \pi)^{1-\epsilon}}\left[T \sum_{\omega_{n} \neq 0} \frac{1}{c^{2} k^{2}+\omega_{n}^{2}}-\int \frac{d \omega}{2 \pi} \frac{1}{c^{2} k^{2}+\omega^{2}+T^{2}}\right] \\
& +c^{1-\epsilon} \int \frac{d^{2-\epsilon} p}{(2 \pi)^{1-\epsilon}} \frac{1}{c^{2} p^{2}+T^{2}} \\
& =\frac{1}{c}\left(\frac{T}{c}\right)^{-\epsilon}\left\{\int \frac{d^{1-\epsilon} k}{(2 \pi)^{1-\epsilon}}\left[\frac{1}{2 k} \operatorname{coth} \frac{k}{2}-\frac{1}{k^{2}}-\frac{1}{2 \sqrt{k^{2}+1}}\right]\right. \\
& \left.+\frac{\Gamma(\epsilon / 2)}{(4 \pi)^{1-\epsilon / 2}}\right\}
\end{aligned}
$$

We are only interested in the poles in $\epsilon$ and the accompanying constants, and to this accuracy the first integral on the right hand side can be evaluated directly at $\epsilon=0$, while the $\Gamma$ function yields a pole. Now combining (4.6), (4.7) and (4.8) into (4.5) we find that the poles in $\epsilon$ cancel (as they must), and

$$
\frac{(N-1) \xi T}{2 c}=\frac{1}{g_{R}(\mu)}-\frac{(N-2)}{2 \pi} \ln (c \mu / T \sqrt{\mathcal{G}})
$$

where the constant $\mathcal{G}$ is

$$
\mathcal{G}=4 \pi e^{-\gamma}=7.055507955 \ldots
$$

with $\gamma$ Euler's constant. Now we use the conventional relationship between $\mu$ and the renormalization group invariant $\Lambda_{\overline{M S}}[55,53$

$$
\Lambda_{\overline{M S}}=\mu \sqrt{\mathcal{G}}\left(\frac{(N-2)}{2 \pi} g_{R}(\mu)\right)^{-1 /(N-2)} \exp \left(-\frac{2 \pi}{(N-2) g_{R}(\mu)}\right)
$$

to eliminate the scale $\mu$ from (4.9). As expected, the coupling $g_{R}(\mu)$ drops out of the resulting expression, and we get

$$
\begin{aligned}
& \xi=\frac{c(N-2)}{T \pi(N-1)}\left\{\ln \left[\frac{\mathcal{G} T}{c \Lambda_{\overline{M S}}}\right]\right. \\
&\left.+\frac{1}{(N-2)} \ln \ln \frac{T}{c \Lambda_{\overline{M S}}}+\mathcal{O}\left(\frac{\ln \ln \left(T / c \Lambda_{\overline{M S}}\right)}{\ln \left(T / c \Lambda_{\overline{M S}}\right)}\right)\right\} .
\end{aligned}
$$


Finally, we can express this in terms of the $T=0$ gap $\Delta$ by using the relationship between $\Lambda_{\overline{M S}}$ and $\Delta$ obtained using the Bethe ansatz solution of the $\sigma$-model [56]

$$
\frac{\Delta}{c \Lambda_{\overline{M S}}}=\frac{(8 / e)^{1 /(N-2)}}{\Gamma(1+1 /(N-2))} .
$$

The results (4.12,4.13) lead to the $N=3$ result for $\xi$ quoted earlier in (1.8).

Having obtained the classical model (4.4) for the equal-time correlations, and the precise value of the coupling $\xi$ in (4.12), we now turn to an examination of unequal time correlations in the high $T$ region $T \gg \Delta$. We employ an approach related to that used in the study of the quantum $\sigma$-model in $d=2$ in Ref [57] in a low $T$ region; unlike (4.4), the equal time correlations in $d=2$ were described by a theory that was not ultraviolet finite, and this will lead to significant differences in the analysis and physical properties here. To obtain classical equations of motions we clearly need to extend the classical Hamiltonian in (4.4) by including a kinetic energy term, expressed in terms of a canonical conjugate momentum to $n_{\alpha}$. The obvious approach is to take the quantum equations of motion, and to simply treat the variables as $c$-number classical degrees of freedom. In particular, we treat the rotor-angular momentum $L$ as a classical variable, and augment the classical Hamiltonian by the kinetic energy of rotational motion. The moment of inertia of the rotor is related to the response of the system to a magnetic field $H$, and we therefore need to study the behavior of $\chi_{u}$ in the $T \gg \Delta$ regime.

We will determine $\chi_{u}$ by strategy similar to that employed above in the computation of $\xi$ : first integrate out the non-zero frequency modes, and then perform the average over the zero frequency fluctuations. We choose an $H$ which rotates $n_{\alpha}$ in the 1-2 plane, and define

$$
n_{\alpha}(x, \tau)=\sqrt{1-\vec{\pi}^{2}(x, \tau)} n_{\alpha}(x)+\sum_{a=1}^{N-1} \pi_{a}(x, \tau) e_{a \alpha}(x)
$$

where $n_{\alpha}(x), e_{a \alpha}(x)$ are a set of $N$ mutually orthogonal vectors in spacetime, and $\pi_{a}(x, \tau)$ represent the finite frequency degrees of freedom which must be integrated out. We expand the partition function to quadratic order in $H$, drop all terms proportional to the spatial gradients of $n_{\alpha}(x)$ or $e_{a \alpha}(x)$ (these can be shown to be logarithmically subdominant to the terms kept), and find that the $H$ dependent terms in the free energy density are

$$
\begin{aligned}
& -\frac{H^{2}}{2 c g}\left[\left(n_{1}^{2}+n_{2}^{2}\right)\left(1-\sum_{a}\left\langle\pi_{a}^{2}\right\rangle\right)+\sum_{a b}\left(e_{a 1} e_{b 1}+e_{a 2} e_{b 2}\right)\left\langle\pi_{a} \pi_{b}\right\rangle\right. \\
& \left.-\frac{1}{c g} \sum_{a b c d}\left(e_{a 1} e_{b 2}-e_{a 2} e_{b 1}\right)\left(e_{c 1} e_{d 2}-e_{c 2} e_{d 1}\right) \int d x d \tau\left\langle\pi_{a} \partial_{\tau} \pi_{b}(0,0) ; \pi_{c} \partial_{\tau} \pi_{d}(x, \tau)\right\rangle\right]
\end{aligned}
$$

Evaluating the expectation values of the $\pi$ fields, and using orthonormality of the vectors $n_{\alpha}, e_{a \alpha}$, the expression (4.15) simplifies to

$$
\begin{aligned}
& -\frac{H^{2}}{2 c g}\left[\left(n_{1}^{2}+n_{2}^{2}\right)\left(1-c(N-2) g T \sum_{\omega_{n} \neq 0} \int \frac{d k}{2 \pi} \frac{1}{c^{2} k^{2}+\omega_{n}^{2}}\right)\right. \\
& \left.+2 c g\left(1-n_{1}^{2}-n_{2}^{2}\right) T \sum_{\omega_{n} \neq 0} \int \frac{d k}{2 \pi} \frac{c^{2} k^{2}-\omega_{n}^{2}}{\left(c^{2} k^{2}+\omega_{n}^{2}\right)^{2}}\right]
\end{aligned}
$$


Finally to obtain the susceptibility $\chi_{u}$, we have to evaluate the expectation value of the zero frequency field $n_{\alpha}$ under the partition function (4.4). This simply yields $\left\langle n_{1}^{2}\right\rangle=\left\langle n_{2}^{2}\right\rangle=1 / N$. The first frequency summation is precisely the same as that evaluated earlier for $\xi$ in (4.5), while the second is explicitly finite in $d=1$ and can directly evaluated; in this manner we obtain our final result for $\chi_{u}$ :

$$
\begin{aligned}
\chi_{u}(T) & =\frac{2}{N}\left[\frac{(N-1) T \xi}{2 c^{2}}-\frac{(N-2)}{2 \pi c}\right] \\
& =\frac{(N-2)}{N \pi c} \ln \left(\frac{\mathcal{G} T}{\Lambda_{\overline{M S}} e}\right)
\end{aligned}
$$

We have omitted the form of the subleading logarithms, which are the same as those in (4.12). This result was quoted earlier in (1.9).

We have now assembled all the information necessary to describe the effective classical dynamics in the region $T \gg \Delta$. The classical partition function is given by the following phase-space functional integral, which generalizes (4.4) (and we will now specialize the remainder of the discussion to the special case $N=3$ ):

$$
\begin{aligned}
& Z=\int \mathcal{D} n_{\alpha}(x) \mathcal{D} L_{\alpha}(x) \delta\left(n_{\alpha}^{2}-1\right) \delta\left(L_{\alpha} n_{\alpha}\right) \exp \left(-\frac{\mathcal{H}_{c}}{T}\right) \\
& \mathcal{H}_{c}=\frac{1}{2} \int d x\left[T \xi\left(\frac{d n_{\alpha}}{d x}\right)^{2}+\frac{1}{\chi_{u \perp}} L_{\alpha}^{2}\right]
\end{aligned}
$$

where $L_{\alpha}$ is the classical angular momentum density, and $L_{\alpha}, n_{\alpha}$ are classical commuting variables. The second term in $\mathcal{H}_{c}$ was absent in (4.4), and represents the kinetic energy of the classical rotors: integrating out $L_{\alpha}$ we obtain (4.4). By evaluating linear response to a field under which

$$
\mathcal{H}_{c} \rightarrow \mathcal{H}_{c}-\int d x H_{\alpha} L_{\alpha}
$$

we find

$$
\chi_{u}=\frac{2}{N} \chi_{u \perp}
$$

with $N=3$ (we have given, without proof, the expression for general $N$ ); the factor of $2 / 3$ comes from the contraint $L_{\alpha} \cdot n_{\alpha}=0$. Using (4.17), we then have the value of $\chi_{u \perp}$.

We can finally specify the manner in which time-dependent correlations have to be computed in this effective classical model. The classical equations of motion are the HamiltonJacobi equations of the Hamiltonian $\mathcal{H}_{c}$, with Poisson brackets which are the continuum classical limit of the quantum commutation relations :

$$
\begin{aligned}
& \left\{L_{\alpha}(x), L_{\beta}\left(x^{\prime}\right)\right\}_{P B}=\epsilon_{\alpha \beta \gamma} L_{\gamma}(x) \delta\left(x-x^{\prime}\right) \\
& \left\{L_{\alpha}(x), n_{\beta}\left(x^{\prime}\right)\right\}_{P B}=\epsilon_{\alpha \beta \gamma} n_{\gamma}(x) \delta\left(x-x^{\prime}\right) \\
& \left\{n_{\alpha}(x), n_{\beta}\left(x^{\prime}\right)\right\}_{P B}=0 .
\end{aligned}
$$

The equations of motion are 


$$
\begin{aligned}
\frac{\partial n_{\alpha}}{\partial t} & =\left(\frac{1}{\chi_{u \perp}}\right) \epsilon_{\alpha \beta \gamma} L_{\beta} n_{\gamma} \\
\frac{\partial L_{\alpha}}{\partial t} & =(T \xi) \epsilon_{\alpha \beta \gamma} n_{\beta} \frac{\partial^{2} n_{\gamma}}{\partial x^{2}}
\end{aligned}
$$

The classical correlation functions are obtained by averaging these deterministic equations over an ensemble of initial conditions specified by (4.18). Note also that simple dimensional analysis of the differential equations (4.22) shows that disturbances travel with a characteristic velocity $c(T)$ given by

$$
c(T)=\left(T \xi(T) / \chi_{u \perp}(T)\right)^{1 / 2},
$$

Notice from (4.12) and (4.17) that to leading logarithms $c(T) \approx c$, but the second term in the first equation of (4.17) already shows that exact equality does not hold.

We complete the relationship of the quantum to the classical model, by noting that there is also an additional wave-function renormalization of the $n_{\alpha}$ field 47,55 which appears when the non-zero frequency modes are integrated out. Our final result for the correlator $C$ in (3.3) then takes the form

$$
C_{\alpha \beta}(x, t)=\mathcal{A} \widetilde{\mathcal{G}}\left[\ln \left(\frac{T}{\Delta}\right)\right]^{\frac{(N-1)}{(N-2)}}\left\langle n_{\alpha}(x, t) n_{\beta}(x, t)\right\rangle_{c}
$$

The subscript $c$ represents the classical average specified by (4.18) and (4.22). The constant $\mathcal{A}$ is the $T=0$ quasi-particle residue which appeared in (3.5). The constant $\widetilde{\mathcal{G}}$ is an unknown universal number which cannot be obtained by the present methods. It could, in principle, be obtained from the Bethe-ansatz solution. There is no similar renormalization of the correlator of the magnetization density , $C_{u}$ in (3.14), which is precisely equal to the two-point correlator of $L_{\alpha}$ under (4.18) and (4.22).

It is now possible to perform a simple rescaling and to show that the classical dynamics problem above is free of any dimensionless couplings, and is a unique, parameter-free theory. This will allow us to completely specify the $T$ dependence of observables upto unknown numerical constants. Let us perform the following rescalings on (4.18) and (4.22)

$$
\begin{aligned}
x & =\bar{x} \xi \\
t & =\bar{t} \sqrt{\frac{\xi \chi_{u \perp}}{T}} \\
L_{\alpha} & =\bar{L}_{\alpha} \sqrt{\frac{T \chi_{u \perp}}{\xi}}
\end{aligned}
$$

Then the partition function (4.18) is transformed to

$$
\begin{aligned}
& \bar{Z}=\int \mathcal{D} n_{\alpha}(\bar{x}) \mathcal{D} \bar{L}_{\alpha}(\bar{x}) \delta\left(n_{\alpha}^{2}-1\right) \delta\left(\bar{L}_{\alpha} \cdot n_{\alpha}\right) \exp \left(-\mathcal{H}_{\bar{c}}\right) \\
& \mathcal{H}_{\bar{c}}=\frac{1}{2} \int d \bar{x}\left[\left(\frac{d n_{\alpha}}{d \bar{x}}\right)^{2}+\bar{L}_{\alpha}^{2}\right]
\end{aligned}
$$


while the equations of motion become

$$
\begin{aligned}
\frac{\partial n_{\alpha}}{\partial \bar{t}} & =\epsilon_{\alpha \beta \gamma} \bar{L}_{\beta} n_{\gamma} \\
\frac{\partial \bar{L}_{\alpha}}{\partial \bar{t}} & =\epsilon_{\alpha \beta \gamma} n_{\beta} \frac{\partial^{2} n_{\gamma}}{\partial \bar{x}^{2}} .
\end{aligned}
$$

Notice that coupling constants and parameters have been scaled away, and (4.26,4.27) constitute a unique problem that must be solved exactly. The $T$ and $\Delta$ dependencies of all quantities arise only through the rescalings defined in (4.25) and the results (4.12) and (4.17,4.20) for $\xi$ and $\chi_{u \perp}$ given earlier. Complete description of the correlators now requires exact solution of (4.26,4.27). The equal-time correlations are of-course known from (4.26):

$$
\begin{aligned}
\left\langle\bar{L}_{\alpha}(\bar{x}, 0) \bar{L}_{\beta}(0,0)\right\rangle_{\bar{c}} & =\frac{2}{3} \delta_{\alpha \beta} \delta(\bar{x}) \\
\left\langle n_{\alpha}(\bar{x}, 0) n_{\beta}(0,0)\right\rangle_{\bar{c}} & =\frac{1}{3} \delta_{\alpha \beta} e^{-|\bar{x}|}
\end{aligned}
$$

Even though the equations of motion constitute an integrable system with an infinite number of non-local conservation laws [58,59], it is not known how to analytically compute correlations averaged over the initial conditions of a thermal ensemble, or whether the correlator $\left\langle\bar{L}_{\alpha}(\bar{x}, \bar{t}) \bar{L}_{\beta}(0,0)\right\rangle_{\bar{c}}$ has a diffusive form at long times and distances. If diffusion did exist in the continuum equations (4.28), the present analysis does allows us to completely specify the $T$ dependence of the diffusion constant; by a simple dimensional analysis of (4.25), we get

$$
D_{s}=\mathcal{B} \frac{T^{1 / 2}[\xi(T)]^{3 / 2}}{\left[\chi_{u \perp}(T)\right]^{1 / 2}}
$$

where $\mathcal{B}$ is an unknown universal number, and the $T$ dependencies of $\xi$ and $\chi_{u \perp}$ are in (4.12) and $4.17,4.20$ ).

In this context, it is interesting to note that recent measurements 60 of the field dependence of $1 / T_{1}$ in the compound $(\mathrm{VO})_{2} \mathrm{P}_{2} \mathrm{O}_{7}$ at temperatures $T \gg \Delta$ seem to provide clear evidence for spin diffusion. However, the bulk of the data is at temperatures comparable to the microscopic exchange constants of the system and it is not clear if the foregoing description based on the universal high temperature properties of the continuum field theory is applicable in the temperature regime studied experimentally. It is interesting that the experimental results appear to suggest that $D_{s} \sim c \xi$, which is consistent with (4.29) if $\chi_{u} \sim T \xi / c^{2}$ (as is the case with our results (1.8) and (1.9) to leading logarithms).

\section{CONCLUSIONS}

The main results of the paper are already summarized in Section \, and here we will note some unresolved issues and directions for future work.

All experimental realizations of gapped antiferromagnets have additional complications which have not been included in the model systems studied here. Most important among 
these are the spin anisotropies away from perfect Heisenberg symmetry and the inter-chain couplings which make the system only quasi-one-dimensional.

Consider first the consequence of anisotropy. The three-fold degenerate quasiparticle spectrum will now be lifted, and three resulting particles will have have different energy gaps and masses. Further, these parameters will depend in a complicated way upon the external field. Nevertheless, we expect that the simple structure of the $\mathcal{S}$-matrix in (1.2) will be retained, as it only depends upon simple dimensional properties of slowly moving particles with a quadratic dispersion. Correlations of the particle density can probably be computed along the semiclassical lines of this paper: one has to deal with a classical gas of particles of different masses and average densities. The latter problem is considerably more complex than the equal mass case, and there is probably no alternative to numerical simulations. Correlations of the spin operators appear more problematical- these will invariable change the labels of the particles when they act, and therefore lead to differences in the labels in the forward and backward trajectories. Combined with the complication that the masses of the different particles are different, and so their trajectories will have different velocities, we are faced with what appears to be a very complex problem with quantum and classical effects intertwined.

Inter-chain couplings will eventually require us to consider dynamics in two or three dimensions. If temperatures are low enough that the inter-chain motion is coherent, then we have to consider the $\mathcal{S}$-matrix for scattering in higher dimensions. In this case the low-momentum behavior is quite different: in fact the $T$-matrix vanishes at low momenta for $d \geq 2$. We would then expect all scattering to be dominated by elastic scattering of impurities which would control the behavior of the spin diffusion constant and the quasiparticle broadening. On the other hand, systems with only incoherent hopping between chains will probably be dominated by the inelastic scattering along the one-dimensional chains, and display behavior qualitatively similar to that discussed in this paper.

\section{ACKNOWLEDGMENTS}

We are grateful to M. Takigawa for initially stimulating our interest in the issue of spin transport in gapped antiferromagnets by informing us about the convincing experimental evidence for diffusion in Ref [30], and for guiding us through the experimental literature. We thank G. Aeppli, I. Affleck, C. Broholm, G. Chaboussant, V. Korepin, S. Majumdar, B. McCoy, B. Narozhny, B. Normand, D. Reich, T. Senthil and X. Zotos for helpful discussions. This research was supported by the National Science Foundation Grant No. DMR-96-23181.

\section{APPENDIX A: LOCAL CONSERVATION LAWS AND SPIN DIFFUSION}

The computation of the spin diffusivity in Section IIIB was carried out using the exact solution a simple classical model of point particles in one dimension. This model is exactly solvable 49 and possesses an infinite number of local conservation laws, as we will show explicitly below. The existence of spin diffusion then appears to run counter to the conventional wisdom that the time evolution of a integrable system is not 'chaotic' enough to be compatible with diffusion. In particular, one might expect that any non-zero spin current 
produced in the system will not ultimately decay to zero because the numerous conservation laws prevent it. In this appendix we will show that this expectation does not hold for the particular model being studied, and that an important 'particle-hole'-like symmetry allows complete decay of any spin current at $H=0$. In a finite magnetic field $(H \neq 0)$, the particle-hole symmetry is absent, and then the spin current does not decay completely: this is consistent with the presence of a purely ballistic component, $F_{1}$, in (3.19) which contributes only for $H \neq 0\left(A_{1}=0\right.$ at $\left.H=0\right)$, and the arguments of Zotos et al [20]. A closely related particle-hole symmetry also played an important role in the appearance of a finite conductivity in our recent quantum transport analysis in two dimensions [61].

The classical model of Sections [IIA and IIIB consisted of particles $k=1 \ldots N$ with spins $m_{k}$ chosen randomly (at $H=0$ ) from $1,0,-1$. At time $t=0$ the particles had uncorrelated random positions $x_{k}(0)$, and subsequently they occupied 'trajectories' $X_{k}(t) \equiv x_{k}(0)+v_{k} t$ where $v_{k}$ are uncorrelated random velocities chosen from a Boltzmann distribution. The position $x_{k}(t)$ of particle $k$ was however a rather complicated function of time, and was chosen from the set of trajectories, $\left\{X_{k}(t)\right\}$, such that for all $t, x_{k}(t)<x_{l}(t)$ for every $k<l$.

It is useful at this point to note two discrete symmetries of the above classical statistical problem at $H=0$. The first is the time-reversal symmetry, $\mathbf{T}$, under which both spins and velocities change sign:

$$
\mathbf{T}: \quad v_{k} \rightarrow-v_{k} \quad, \quad m_{k} \rightarrow-m_{k} .
$$

The second is the 'particle-hole' symmetry $\mathbf{P}$, under which only the spins reverse direction:

$$
\mathbf{P}: \quad v_{k} \rightarrow v_{k} \quad, \quad m_{k} \rightarrow-m_{k} .
$$

These symmetries will be crucial in our discussion below.

Let us now explicitly identify the local conserved quantities of this classical dynamics. All of the velocities $v_{k}$ are clearly constants of the motion. However, we would like to work with locally conserved quantities which can be written as the spatial integrals over local observables, and which are invariant under permutation of the particle labels; so we define

$$
\begin{aligned}
V_{n} & =\int d x\left[\sum_{k=1}^{N}\left(\frac{d x_{k}(t)}{d t}\right)^{n} \delta\left(x-x_{k}(t)\right)\right] \\
& =\sum_{k=1}^{N} v_{k}^{n}
\end{aligned}
$$

with $n=1 \ldots N$ (notice $d x_{k}(t) / d t \neq v_{k}=d X_{k}(t) / d t$, but the result holds after summation over $k$ because the set $\left\{x_{k}(t)\right\}$ differs from the set $\left\{X_{k}(t)\right\}$ only by a renumbering). All the $V_{n}$ are constants of the motion. Similarly, with spins $m_{k}$ we can define

$$
M_{p}=\sum_{k=1}^{N} m_{k}^{p}
$$

with $p=1,2$, as additional locally conserved quantities ( $M_{1}$ is the spatial integral of $\varrho_{z}(x, t)$ in (3.15), and a similar result holds of $M_{2}$ ). We can now easily work out the signature of the $V_{n}$ and $M_{p}$ under the discrete symmetries noted earlier, and tabulate the results: 


\begin{tabular}{c|c|c} 
& $\mathbf{P}$ & $\mathbf{T}$ \\
\hline$V_{n}, n$ odd & 1 & -1 \\
\hline$V_{n}, n$ even & 1 & 1 \\
\hline$M_{1}$ & -1 & -1 \\
\hline$M_{2}$ & 1 & 1 \\
\hline
\end{tabular}

The central quantity in spin transport is the total spin current $J(t)$, which is not a constant of the motion. It is also given by an integral over a local quantity as

$$
\begin{aligned}
J(t) & =\int d x\left[\sum_{k=1}^{N} m_{k} \frac{d x_{k}(t)}{d t} \delta\left(x-x_{k}(t)\right)\right] \\
& =\sum_{j, k=1}^{N} m_{j} v_{k} A_{j k}(t),
\end{aligned}
$$

where $A_{j k}$ is defined to be equal to 1 if particle $j$ is on trajectory $k$ at time $t$ and 0 otherwise; we will analytically study the function $A_{j k}$ in Appendix C, but here we will be satisfied by a numerical simulation. Again, as in (A5) it is useful to note the signature of $J$ under the discrete symmetries:

\begin{tabular}{l|c|c} 
& $\mathbf{P}$ & $\mathbf{T}$ \\
\hline $\mathrm{J}$ & -1 & 1 \\
\hline
\end{tabular}

As will become clear shortly, one of the central points of this appendix is that the signatures in (A7) differ from all of those of the conserved quantities in (A5). The current $J(t)$ is the sum of $N$ random numbers of each sign, and so is expected to be of order $\sqrt{N}$ for a typical initial condition chosen from the ensemble defined above. We show the deterministic time evolution of $J(t)$ for one such initial condition for a system of 400 particles in Fig 23: notice that it is rather noisy-looking and repeatedly changes its sign. Also, among the constants of the motion above, we expect $V_{n}$ with $n$ odd and $M_{1}$ to be of order $\sqrt{N}$ (provided $n$ is not too large), and $V_{n}$ with $n$ even and $M_{2}$ to be of order $N$ for a typical initial condition; notice that it is only the conserved quantities of order $\sqrt{N}$ that can distinguish left movers from right movers, or spin up from down.

Let us now create a macroscopic spin current (of order $N$ ) in this system. We do this by hitting the system with a magnetic field gradient impulse at a time $t=t_{0}$, and subsequently setting the field to zero. As a result of the impulse, the velocities of the particles with spin up are assumed to increase by $v_{0}$, while those of spin down are assumed to decrease by $v_{0}$. Formally, this can be written as

$$
v_{k} \rightarrow v_{k}+m_{l} v_{0} \text { where } l \text { is unique solution of } A_{l k}\left(t_{0}\right)=1 \text {. }
$$

Immediately after the impulse, $J(t)$ will have a macroscopic value

$$
J\left(t_{0}^{+}\right)=\frac{2}{3} N v_{0}+\mathcal{O}(\sqrt{N})
$$

The subsequent deterministic time evolution of $J(t)$ is also shown in Fig 23: it decays in a few collision times to a value of order $\sqrt{N}$ and then appears to chaotically oscillate in time! 
The basic point is now easy to see. Because $m_{k}$ is as likely to be +1 or -1 , the impulse on any given particle is equally likely to be $+v_{0}$ or $-v_{0}$. Hence the $V_{n}$, with $n$ odd, remain of order $\sqrt{N}$ even after the impulse. This is simply a manifestation of the fact that the signatures of $J$ under $\mathbf{P}$ and $\mathbf{T}$ are different from those of the $V_{n}$. A non-zero $J$ is therefore not correlated with an induced value of a conserved quantity which could prevent the decay of $J$ to non-macroscopic values.

\section{APPENDIX B: NUMERICAL COMPUTATION OF THE FOURIER TRANSFORM OF THE CORRELATION FUNCTION $C$}

In this appendix we outline the numerical method employed in calculating $S(q, \omega)$ starting from the numerically determined semiclassical $C(x, t)$ and the procedure used to directly determine the scaling function $\Phi(z)$ (see Eqn (3.12))

As the numerical determination of $\tilde{R}(\tilde{x}, \tilde{t})$ is the most time consuming part of the entire procedure, we calculated $\tilde{R}$ only at a predetermined grid of points in the $\tilde{x}-\tilde{t}$ plane. We chose $\tilde{t}$ values from 0 to 7.0 at intervals of 0.2 . For each such value of $\tilde{t}$, we chose about 20 points so as to sample $\tilde{R}$ as well as possible in the region in which $\tilde{R}>5 \times 10^{-3}$; this choice was made to reflect the fact that our absolute error in $\tilde{R}$ was estimated to be about $5 \times 10^{-4}$. This then defined our grid. At each $\tilde{t}$, we fit $\tilde{R}$ as a function of $\tilde{x}$ to the form

$$
\log (\tilde{R})=-\frac{a_{1} a_{2}+a_{3} \tilde{x}+a_{4} \tilde{x}^{2}+f \tilde{x}^{3}}{a_{2}+a_{5} \tilde{x}+\tilde{x}^{2}},
$$

where $f=4 / 3$ and $a_{1}=-\log (\tilde{R}(0, \tilde{t}))$. The rationale behind our choice of the value of $f$ is as follows: When $\tilde{x} \gg \tilde{t}$, the complicated correlations between the spin labels of a given classical trajectory at different times do not matter and $R$ is well approximated by our 'mean-field' theory (see Appendix D). The mean-field theory in this limit gives $\log (\tilde{R}) \sim-4 \tilde{x} / 3$ and this is what determines our choice of $f$. The error in the fit was estimated to be roughly the same as the error in the original computation of $\tilde{R}$; thus we did not lose anything by doing the fit. Having tabulated the fitting parameters for each value of $\tilde{t}$ on the grid, we evaluated the spatial Fourier transform numerically. The resulting function of $\tilde{t}$ is expected to be smooth as long as $\delta \tilde{\omega}=(\omega-\varepsilon(k)) L_{t}$ is not too large. More precisely, we do not expect any oscillations on the scale of our grid spacing in $\tilde{t}$ as long as $0.2 \delta \tilde{\omega} \ll 2 \pi$. As we are interested only in $\delta \tilde{\omega} \sim 1$, we can safely interpolate the resulting function in $\tilde{t}$. In practice we use a cubic-spline to do the interpolation. Lastly, we do the $\tilde{t}$ integral numerically to obtain $S(q, \omega)$. The accuracy of both numerical integrations is quite high and so we expect that the dominant error in our calculation comes from the interpolation; this is conservatively estimated to be a few percent at the most for the largest values of $\delta \tilde{\omega}$.

Let us now briefly indicate the procedure used in obtaining the Fourier transform of $\tilde{R}(0, \tilde{t})$ needed for the calculation of the scaling function $\Phi(z)$. The available data for $\tilde{R}(0, \tilde{t})$ is fit extremely well by the following form:

$$
\log (\tilde{R})=-\frac{\alpha \tilde{t}+a \tilde{t}^{2}+b \tilde{t}^{3}}{1+c \tilde{t}+d \tilde{t}^{2}},
$$


where the choice $\alpha=4 / 3 \sqrt{\pi}$ is again motivated by 'mean-field' considerations. It is now a simple matter to do the Fourier integral to a very high accuracy using this fit and we estimate the errors involved to be less than $0.5 \%$ at the most.

\section{APPENDIX C: CALCULATION OF TAGGED PARTICLE CORRELATIONS IN THE CLASSICAL MODEL}

In this appendix, we shall attempt to give a self-contained account of the method devised by Jepsen 49 for the calculation of the tagged particle correlations in the classical model introduced in Ref [49]. We will try to adhere to the notation and conventions of [49] as far as possible.

The model is defined as follows: We begin with $N$ particles of mass $m$ distributed uniformly along a one-dimensional segment of length $L$ with periodic boundary conditions (we will eventually take the thermodynamic limit $L \rightarrow \infty$ with $N / L$ fixed to be equal to the density $\rho$ ). At time $t=0$ each particle is assigned a velocity from the classical thermal ensemble defined by the usual Maxwell-Boltzmann distribution function $g(v)=$ $(m / 2 \pi T)^{1 / 2} e^{-m v^{2} / 2 T}$. The subsequent evolution of the system is purely deterministic; the particles travel without any change in their velocities until they collide with another particle. Every collision is elastic and the particles merely exchange their velocities as a result of the collision.

To begin our analysis, let us label the particles from left to right with an index $i$ running from 0 to $N-1$. Thus the particles are initially at positions $x_{i}(0)$ such that $x_{i}(0)<x_{j}(0)$ for $i<j$. Actually, it is convenient to identify $i+N$ with $i$ because of the periodic boundary conditions employed which identify the ends $x=0$ and $x=L$ of the interval. Note that this labelling of the particles is left invariant by the dynamics. We also label trajectories (which follow the straight line defined by $X_{i}(t)=x_{i}(0)+v_{i} t$ on the space-time diagram representing the evolution of the system) with an index $i$, again with the convention that $x_{i}(0)<x_{j}(0)$ for $i<j$ (here $v_{i}$ is the initial velocity of the $i^{t h}$ particle). Let $x_{i}(t)$ denote the position of the $i^{\text {th }}$ particle at time $t$. We wish to calculate the correlator $B(x, t)=\left\langle\delta\left(x-x_{k}(t)\right) \delta\left(x_{k}(0)\right)\right\rangle$ where summation over the repeated index $k$ is implied and the angular brackets refer to averaging over the ensemble of initial conditions specified earlier.

Let us now consider the quantity $A_{j k}(t)$, introduced in Appendix A, which is defined to be equal to 1 if particle $j$ is on trajectory $k$ at time $t$ and 0 otherwise. Another useful quantity is the number $n_{k}$ of (signed) crossings suffered by the $k^{\text {th }}$ trajectory upto time $t$. Every time this trajectory is hit from the left, $n_{k}$ decreases by 1 and every time it is hit from the right $n_{k}$ increases by 1 . Clearly, $A_{j k}(t)=1$ for $j=k+n_{k}(t)$ and zero otherwise. We may probe the dynamics a bit more by defining another quantity $r_{n}(h, k, t)$ which equals 1 if trajectory $h$ has crossed trajectory $k$ precisely $n$ times upto time $t$ and zero otherwise. Here too, we are talking of signed crossings; if trajectory $h$ crosses from the left this is a negative crossing and if it crosses from the right it is a positive crossing. Clearly $r_{n}$ has the interpretation of a probability when averaged over any ensemble of initial conditions. Let us also define the corresponding 'generating function' as

$$
s(u ; h, k, t)=\sum_{n=-\infty}^{\infty} r_{n}(h, k, t) e^{i n u} .
$$


The reason for introducing $r_{n}$ and $s(u)$ is that $A_{j k}(t)$, which is clearly a central quantity of interest, may be very conveniently expressed in terms of $s(u)$ as

$$
A_{j k}(t)=\frac{1}{N} \sum_{l=0}^{N-1} e^{-\frac{2 \pi i}{N}(j-k) l} \prod_{m=0}^{N-1} s\left(\frac{2 \pi l}{N} ; m, k, t\right)
$$

here we are using the convention that $s(u ; k, k, t) \equiv 1$. This is quite easy to check from the defintions of $s(u)$ and $A_{j k}$. Moreover, it is possible to write down a fairly explicit expression for $s(u ; h, k, t)$. This takes a slightly different form depending on whether $h$ is greater or less than $k$. If $h>k$, we have

$$
s(u ; h, k, t)=S\left[u, w_{k h}\right]
$$

while if $h<k$, we have

$$
s(u ; h, k, t)=e^{-i u} S\left[u, w_{k h}\right] .
$$

$S\left[u, w_{k h}\right]$ used above is defined as:

$$
S\left[u, w_{k h}\right]=e^{i n u},
$$

where $w_{k h} \equiv x_{k}(0)-x_{h}(0)+\left(v_{k}-v_{h}\right) t$, and $n$ is the integer that satisfies $(n-1) L<w_{k h}<n L$. Using this definition, we can write the following compact expression for $A_{j k}(t)$ in terms of $S$ :

$$
A_{j k}(t)=\frac{1}{N} \sum_{l=0}^{N-1} e^{-\frac{2 \pi i l j}{N}} \prod_{h=0}^{N-1} S\left[\frac{2 \pi l}{N}, w_{k h}\right] .
$$

With all this machinery in place, it is a relatively straightforward matter to calculate the correlation function we need. We begin by explicitly writing out the ensemble averages involved:

$$
B(x, t)=\frac{N !}{L^{N}} \int\{d x\} \int[d v]\left(\prod_{l=0}^{N-1} g\left(v_{l}\right)\right) \delta\left(x_{0}(t)-x\right)
$$

where we have used the definitions $\int\{d x\} \equiv \int_{0}^{L} d x_{N-1} \int_{0}^{x_{N-1}} d x_{N-2} \ldots \int_{0}^{x_{2}} d x_{1}$ and $\int[d v] \equiv$ $\int_{-\infty}^{\infty} d v_{N-1} \int_{-\infty}^{\infty} d v_{N-2} \ldots \int_{-\infty}^{\infty} d v_{0}$ with $x_{k}(0) \equiv x_{k}$, and it is understood that $x_{0}(0)$ is set equal to 0 when evaluating the right hand side of (C7). Now we can transform from particle positions to trajectories by writing

$$
\delta\left(x_{0}(t)-x\right)=\sum_{k} A_{0 k} \delta\left(X_{k}(t)-x\right) .
$$

Using this and writing $A_{0 k}$ in terms of $S\left[u, w_{k h}\right]$ allows us to express our correlation function as

$$
B(x, t)=\frac{N}{L^{N}} \int[d x] \int[d v]\left(\prod_{l=0}^{N-1} g\left(v_{l}\right)\right) \frac{1}{N} \sum_{k, l=0}^{N-1} \prod_{h=0}^{N-1} S\left[\frac{2 \pi l}{N}, w_{k h}\right] \delta\left(X_{k}(t)-x\right) .
$$


Here we have also used the fact that the integrand in this representation is explicitly symmetric in the spatial integration variables to change the spatial integration to $\int[d x] \equiv \int_{0}^{L} d x_{N-1} \int_{0}^{L} d x_{N-2} \ldots \int_{0}^{L} d x_{1}$.

It is now convenient to define $R\left[u, x_{k}+v_{k} t-x_{h}\right] \equiv \int_{-\infty}^{\infty} d v_{h} g\left(v_{h}\right) S\left[u, w_{k h}\right]$. Using this we can rewrite our expression for the correlation function as

$$
\begin{aligned}
& B(x, t)= \frac{\rho}{N} \sum_{u}\left[\int d v_{0} g\left(v_{0}\right) \delta\left(X_{0}(t)-x\right)\left(\frac{1}{L} \int_{0}^{L} d x_{h} R\left(u, X_{0}(t)-x_{h}\right)\right)^{N-1}\right. \\
&+\frac{N-1}{L} \int_{0}^{L} d x_{k} \int_{-\infty}^{\infty} d v_{k} g\left(v_{k}\right) R\left(u, X_{k}(t)\right) \delta\left(X_{k}(t)-x\right) \\
&\left.\times\left(\frac{1}{L} \int_{0}^{L} d x_{h} R\left(u, X_{k}(t)-x_{h}\right)\right)^{N-2}\right]
\end{aligned}
$$

where $k \neq h, k, h \neq 0, u \equiv 2 \pi l / N$ and $\sum_{u} \equiv \sum_{l=0}^{N-1}$. To proceed further we need to work out $R\left[u, X_{k}(t)\right]$ and $(1 / L) \int_{0}^{L} d x_{h} R\left[u, X_{k}(t)-x_{h}\right]$. This is quite straightforward to do in the limit of large $L$ and we only give the final results below:

$$
\begin{aligned}
R\left[u, X_{k}(t)\right] & =\frac{1}{2} E_{c}(y)+\left(1-\frac{1}{2} E_{c}(y)\right) e^{i u}, \\
\frac{1}{L} \int_{0}^{L} d x_{h} R\left[u, X_{k}(t)-x_{h}\right] & =1+\frac{1}{L}\left(1-e^{-i u}\right) T\left[u, X_{k}(t)\right],
\end{aligned}
$$

where

$$
\begin{aligned}
T\left[u, X_{k}(t)\right] & =\sqrt{\frac{2 T}{m}} t\left[y e^{i u}+\frac{e^{i u}-1}{2}\left(\frac{1}{\sqrt{\pi}} e^{-y^{2}}-y E_{c}(y)\right)\right], \\
E_{c}(y) & =\frac{2}{\sqrt{\pi}} \int_{y}^{\infty} d z e^{-z^{2}}, \\
y & =\sqrt{\frac{m}{2 T}} \frac{X_{k}(t)}{t} .
\end{aligned}
$$

Now, in the the thermodynamic limit specified earlier we can write

$$
\left(1+\frac{1}{L}\left(1-e^{-i u}\right) T\left[u, X_{k}(t)\right]\right)^{N-\nu}=\exp \left(\rho\left(1-e^{-i u}\right) T\left[u, X_{k}(t)\right]\right)
$$

valid for any finite number $\nu$. Using this and (C10) in the expression (C9) for the correlation function and doing the remaining integrals over positions and velocities gives us

$$
\begin{gathered}
B(x, t)=\rho \int_{0}^{2 \pi} \frac{d u}{2 \pi}\left[\rho\left(2 f_{1}(w) f_{2}(w)+e^{i u} f_{2}^{2}(w)+e^{-i u} f_{1}^{2}(w)\right) \exp \left(\rho\left(1-e^{-i u}\right) T[u, x]\right)\right. \\
\left.+\frac{1}{t} \sqrt{\frac{m}{2 \pi T}} e^{-w^{2}} \exp \left(\rho\left(1-e^{-i u}\right) T[u, x]\right)\right] ;
\end{gathered}
$$

here we have replaced the sum over $u$ by the corresponding integral in the thermodynamic limit and used the definitions $f_{1}(w) \equiv E_{c}(w) / 2, f_{2}(w) \equiv 1-f_{1}(w)$ and $w \equiv(m / 2 T)^{1 / 2} x / t$. To do the $u$ integral, we note that $T$ may be expressed as $G e^{i u}-A$ where $A$ and $G$ are functions purely of $x$ and $t$. This allows us to use the standard Bessel function identity, 


$$
\frac{1}{2 \pi} \int_{0}^{2 \pi} d u e^{-i n u} \exp \left(\rho\left(1-e^{-i u}\right)\left(G e^{i u}-A\right)\right)=\left(\frac{G}{A}\right)^{\frac{n}{2}} e^{-\rho(A+G)} I_{n}(2 \rho \sqrt{A G}),
$$

to finally arrive at the results quoted in (3.21) of Section [IIB upon using the appropriate values for $\rho$ and $m$.

\section{APPENDIX D: APPROXIMATE ANALYTICAL CALCULATION OF THE RELAXATION FUNCTION}

In this appendix, we briefly outline our approximate 'mean-field' theory for the relaxation function $R(x, t)$.

We begin by noting that the classical model defined in Section IIIA has been solved exactly in Ref [23] for the special case in which there is only one possible value for the spin label $m$. All of the difficulties we encounter in attempting to generalize this solution to the case of interest here stem from the fact that there are complicated correlations between the $m_{k}(t)$ (defined in Section IIIA) at different times.

Our 'mean-field' approximation consists of simply ignoring these correlation effects (hence our choice of terminology to describe our approximation). Having made this uncontrolled approximation, it is now a fairly straightforward matter to obtain a closed form expression for $R(x, t)$ in analogy with the corresponding discussion in [23]. The actual calculation proceeds as follows: Let $q$ be the probability that any given solid line in Fig 9 intersects the dotted line. If we ignore the correlations between the $m_{k}(t)$ at different times, then the probability that this line carries a spin label equal to the spin label of the dotted line is $1 / 3$. So given that the line intersects the dotted line, this intersection contributes a factor of -1 to $R(x, t)$ with probability $1 / 3$ and a factor of 0 with probability $2 / 3$ (if the line does not intersect at all, we of course get a factor of 1 ). Within our mean field theory, $R$ is just a product of such factors, one from each solid line. This gives $R(x, t)=(1-q-q / 3)^{N}$, where $N$ is the total number of thermally excited particles in the system. Now, using $q=\langle|x-v t|\rangle / L$ [23] (where the angular brackets denote averaging over the MaxwellBoltzmann distribution function for $v$ and $L$ is the length of the system) and taking the thermodynamic limit, we obtain $R(x, t)=\exp (-4 \rho\langle|x-v t|\rangle / 3)$. We can now do our usual rescalings and write down the main result of our mean field theory:

$$
\tilde{R}(\tilde{x}, \tilde{t})=\exp (-4\langle|\tilde{x}-\tilde{v} \tilde{t}|\rangle / 3)
$$

where the angular brackets now denote averaging over the distribution $\tilde{\mathcal{P}}(\tilde{v})=\frac{1}{\sqrt{\pi}} e^{-\tilde{v}^{2}}$ and $\tilde{x}$ and $\tilde{t}$ are defined as in Section [IIA. In particular, note that this implies $\tilde{R}(0, \tilde{t})=$

$e^{-4|\tilde{t}| / 3 \sqrt{\pi}}$; this turns out to be reasonably accurate for some purposes (see the discussion on the approximate form of the scaling function $\Phi(z)$ in Section [II). 


\section{REFERENCES}

[1] Neutron scattering studies of non-metallic low-dimensional quantum antiferromagnets by C. Broholm in Dynamical properties of unconventional magnetic systems, proceedings of the NATO Advanced Study Institute, Geilo, Norway, April 2-12, 1997, A. Skjeltorp and D. Sherrington eds., Kluwer Academic.

[2] The excitations of one-dimensional, spin 1/2 antiferromagnets by R. A. Cowley in Dynamical properties of unconventional magnetic systems, proceedings of the NATO Advanced Study Institute, Geilo, Norway, April 2-12, 1997, A. Skjeltorp and D. Sherrington eds., Kluwer Academic.

[3] G. Chaboussant et al, Report-no: cond-mat 9706138; and references therein.

[4] P. R. Hammar, D. H. Reich, C. Broholm, and F. Trouw, Report-no: cond-mat 9708053.

[5] M. Azuma et al, Phys. Rev. Lett. 73, 3463 (1994).

[6] F. D. M. Haldane Phys. Lett. 93A, 464 (1983).

[7] E. S. Sorensen and I. Affleck, Phys. Rev. B 49, 13235 (1994).

[8] G. Sierra, J. Phys. A 29, 3299 (1996).

[9] A. M. Polyakov, Phys. Lett. B 59, 87 (1975).

[10] A. B. Zamolodchikov and A. B. Zamolodchikov, Ann. of Phys. 120, 253 (1979).

[11] Form factors in completely integrable models of quantum field theory by F.A. Smirnov, World Scientific, Singapore (1992).

[12] See e. g., I. Affleck and R. A. Weston, Phys. Rev. B 45, 4667 (1992).

[13] A. M. Tsvelik, Zh. Eksp. Teor. Fiz. 93, 385 (1987) (Sov. Phys. JETP 66, 221 (1987)).

[14] I. Affleck in Fields, Strings, and Critical Phenomena, edited by E. Brézin and J. ZinnJustin (North-Holland, Amsterdam, 1990), p. 563.

[15] H.J. Schulz, Phys. Rev. B 34, 6372 (1986).

[16] S. Sachdev, Phys. Rev. B 50, 13006 (1994).

[17] O.A. Starykh, R.R.P. Singh, and A.W. Sandvik, Phys. Rev. Lett. 78, 539 (1997).

[18] S. Eggert, I. Affleck, and M. Takahashi, Phys. Rev. Lett. 73, 332 (1994).

[19] B. Narozhny, Phys. Rev. B 54, 3311 (1996).

[20] X. Zotos, F. Naef, and P. Prelovsek, Phys. Rev. B 55, 11029 (1997).

[21] B.M. McCoy, cond-mat/9706194.

[22] S. Sachdev and K. Damle, Phys. Rev. Lett. 78, 943 (1997).

[23] S. Sachdev and A. P. Young, Phys. Rev. Lett. 78, 2220 (1997).

[24] T. Barnes et.al., Phys. Rev. B 47, 3196 (1993); J. Oitmaa, R. R. P. Singh, and Z. Weihong, Phys. Rev. B 54, 1009 (1996).

[25] D. G. Shelton, A. A. Nersesyan, and A. M. Tsvelik, Phys. Rev. B 53, 8521 (1996).

[26] J. Kishine and H. Fukuyama, J. Phys. Soc. of Japan, 66, 26 (1997).

[27] Quantum Inverse Scattering Method and Correlation Functions by V.E. Korepin, N.M. Bogoliubov, and A.G. Izergin, Cambridge University Press, Cambridge (1993), and references therein.

[28] M. Luscher, Nucl. Phys. B135, 1 (1978).

[29] The simplest non-local conserved charge in Ref. [28], $Q^{a b}$, does have the same quantum numbers as the spin current. The discussion in Appendix A would then suggest that $Q^{a b}$ could prevent complete decay of the spin current. However the non-local nature of $Q^{a b}$ suggests to us that this is unlikely. In a system with $N$ thermally excited particles, $Q^{a b}$ 
consists of two components: a local piece with order $N$ terms which is proportional to the spin current at low momentum, and a second non-local piece with order $N^{2}$ terms. In a typical thermal state, the naive expectation is that the value of the local piece will be of order $\sqrt{N}$, while that of the non-local piece will be of order $N$. If we now set up a spin current with an impulse, as in Appendix A, the local piece will jump to a term of order $N$. However this does not change the magnitude of the total value of $Q^{a b}$, and it is reasonable to expect that, within a few collision times, there will be a redistribution within the two pieces of $Q^{a b}$ so that their orders of magnitude are similar to those in a typical thermal state.

[30] M. Takigawa et. al., Phys. Rev. Lett. 76, 2173 (1996).

[31] J. Sagi and I. Affleck, Phys. Rev. B 53, 9188 (1996).

[32] T. Shimizu et. al., Phys. Rev. B 52, R9835 (1995).

[33] Dynamics and transport near quantum-critical points by S. Sachdev in Dynamical properties of unconventional magnetic systems, proceedings of the NATO Advanced Study Institute, Geilo, Norway, April 2-12, 1997, A. Skjeltorp and D. Sherrington eds., Kluwer Academic; report no. cond-mat/9705266.

[34] G.E. Granroth et. al., Phys. Rev. Lett. 77, 1616 (1996).

[35] Th. Jolicoeur and O. Golinelli, Phys. Rev. B 50, 9265 (1994).

[36] A.V. Chubukov, S. Sachdev, and J. Ye, Phys. Rev. B 49, 11919 (1994).

[37] Theory and Experiments on Haldane Gap Antiferromagnets by I. Affleck in Dynamical properties of unconventional magnetic systems, proceedings of the NATO Advanced Study Institute, Geilo, Norway, April 2-12, 1997, A. Skjeltorp and D. Sherrington eds., Kluwer Academic.

[38] S. Sachdev and R. N. Bhatt, Phys. Rev. B 41, 9323 (1990).

[39] G. S. Uhrig and H. J. Schulz, Phys. Rev. B 54, 9624 (1996).

[40] A. W. Garrett et. al., Phys. Rev. B 55, 3631 (1997).

[41] A. W. Garrett et. al., Phys. Rev. Lett. 79, 745 (1997).

[42] E. Dagotto, J. Riera, and D. Scalapino, Phys. Rev. B 45, 5744 (1992); references therein.

[43] D. A. Tennant et. al., Phys. Rev. Lett. 78, 4998 (1997).

[44] D. Beltrán-Porter et. al., Solid State Ionics 32-33, 57 (1989).

[45] Bosonization: How to make it work for you in Condensed Matter by R. Shankar in Current Topics in Condensed Matter and Particle Physics, proceedings of the BCSPIN School, Katmandu, May 1991, J. Pati, Q. Shafi, and Yu Lu eds., World Scientific (1993); also R. Shankar, Acta Phys. Pol. 26, 1835 (1995).

[46] An Introduction to Quantum Field Theory by M. Peskin and D. Schroeder, AddisonWesley (1995).

[47] See e.g. A.M. Polyakov, Gauge Fields and Strings, Chapter 2, Harwood Academic Publishers, New York (1987).

[48] A. N. Kirillov and F. A. Smirnov, Int. J. Mod. Phys. A 3, 731 (1988).

[49] D. W. Jepsen, J. Math. Phys. 6, 405 (1965).

[50] J. L. Lebowitz and J. K. Percus, Phys. Rev. 188, 487 (1969).

[51] K. Damle and S. Sachdev, unpublished.

[52] M. Takigawa et. al., Phys. Rev. B 52, R13087 (1995).

[53] M. Luscher, Phys. Lett. B 118, 391 (1982).

[54] P.C. Hohenberg and B.I. Halperin, Rev. Mod. Phys. 49, 435 (1977). 
[55] E. Brezin and J. Zinn-Justin, Phys. Rev. B 14, 3110 (1976).

[56] P. Hasenfratz and F. Niedermayer, Phys. Lett. B 245, 529 (1990).

[57] S. Tyc, B.I. Halperin, and S. Chakravarty, Phys. Rev. Lett. 62, 835 (1989).

[58] L.D. Faddeev and L.A. Takhtajan, Hamiltonian Methods in the Theory of Solitons, Springer-Verlag, Heidelberg (1987).

[59] K. Pohlmeyer, Comm. Math. Phys. 46, 207 (1976); M. Luscher and K. Pohlmeyer, Nucl. Phys. B137, 46 (1978). The discussion in [29] also applies to the non-local conserved charges, $Q_{2}^{a}$, in the second paper. Also the classical model has the property (not present in the quantum model) that all disturbances travel precisely with the velocity $c(T)$ : this could lead to special singular structure in the correlation functions at $x= \pm c(T) t$.

[60] J. Kikuchi, T. Yamauchi, and Y. Ueda, J. Phys. Soc. Japan 66, 1622 (1997).

[61] K. Damle and S. Sachdev, Phys. Rev. B 56, 8714 (1997). 


\section{FIGURES}

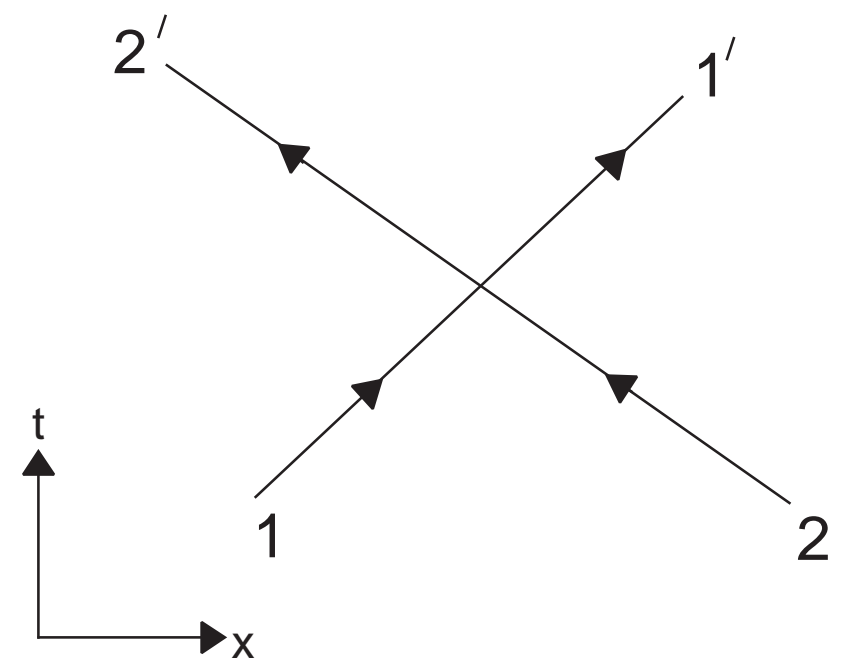

FIG. 1. Two particle collision described by the $\mathcal{S}$-matrix (1.2). The momenta before and after the collision are the same, so the figure also represents the spacetime trajectories of the particles. 


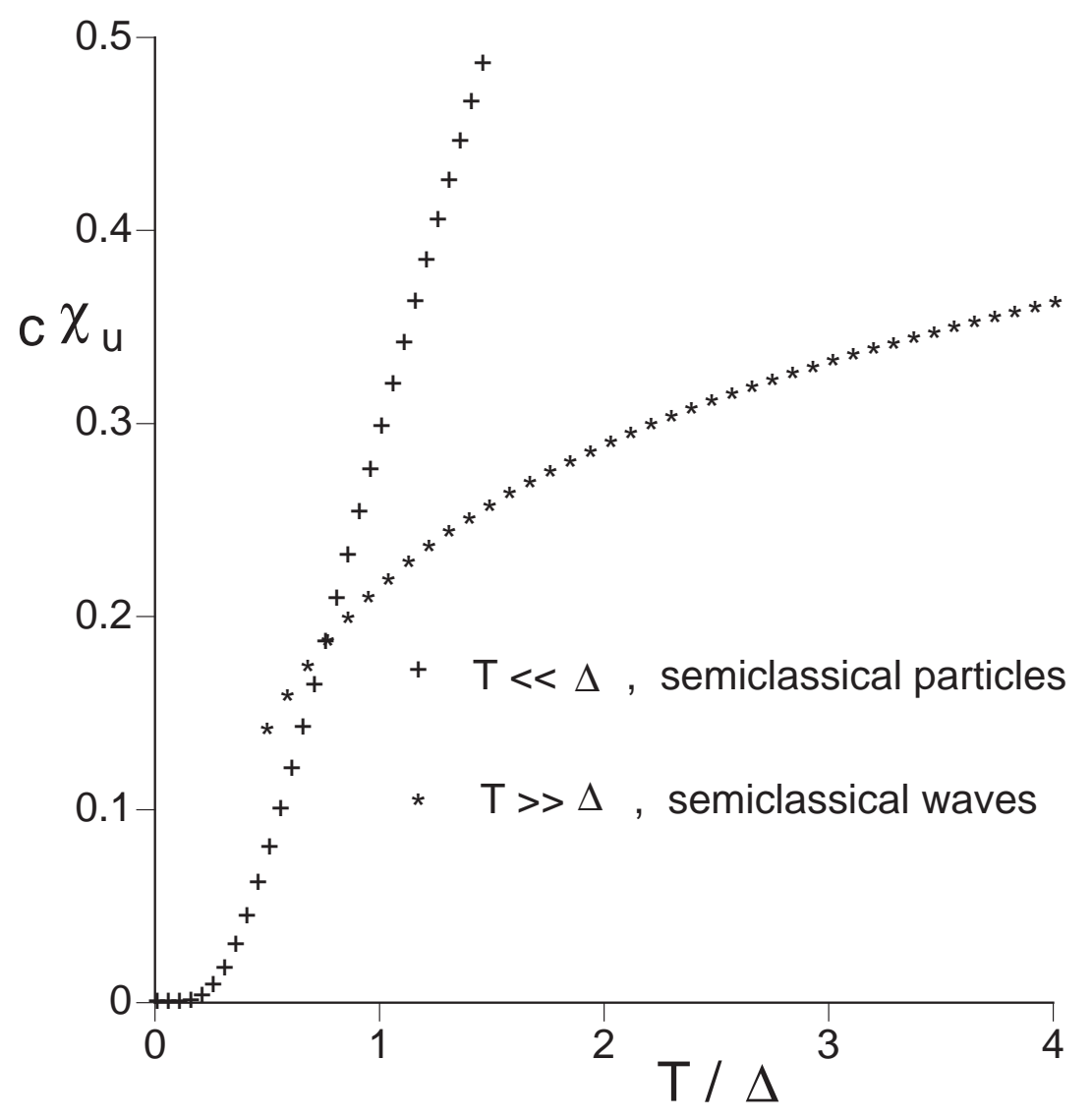

FIG. 2. Low and high temperature asymptotics for the uniform susceptibility $\chi_{u}$ of the continuum $O(3)$ non-linear $\sigma$-model. At $T=0$, there is an energy gap $\Delta$ to all excitations, and $c$ is the velocity defined by (1.1). The expression in Eqn 1.4 gives the low temperature asymptotics while Eqn 1.9 is used for the high temperature asymptotics. Any lattice antiferromagnet will have a very high temperature ( $T>J$ where $J$ is a typical microscopic exchange constant) Curie susceptibility $\sim 1 / T$ which is not shown: the high temperature limit of the continuum theory will apply for $\Delta<T<J$. 


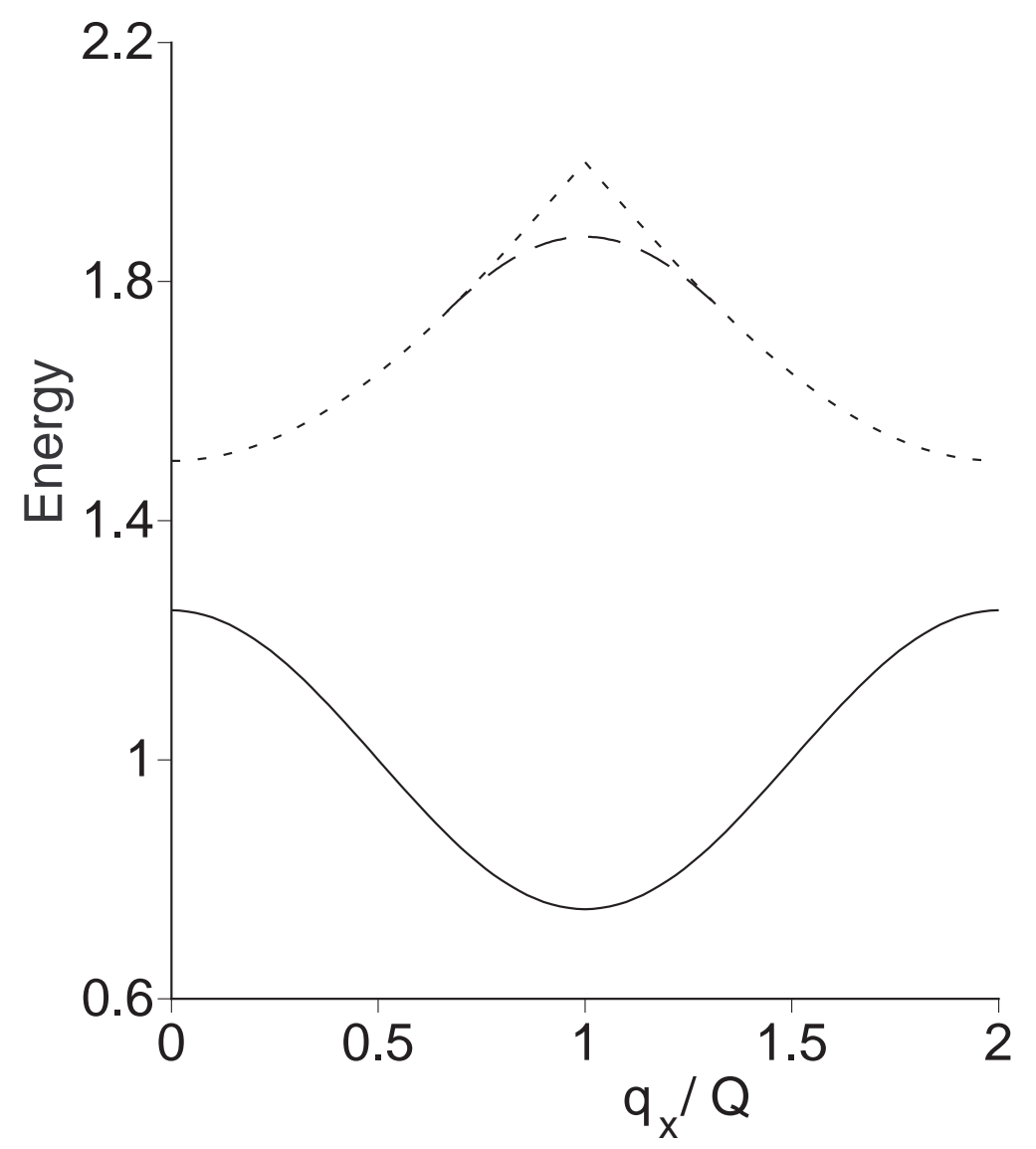

FIG. 3. Positions in $\omega$ of the single particle peak (solid line), bound state peak (long-dash line), and the bottom of the two particle continuum (short-dash line) in $S(\vec{Q}, \omega)$ plotted as a function of $q_{x}$ for the strongly-coupled ladder (a typical value of $g=0.25$ is used). 


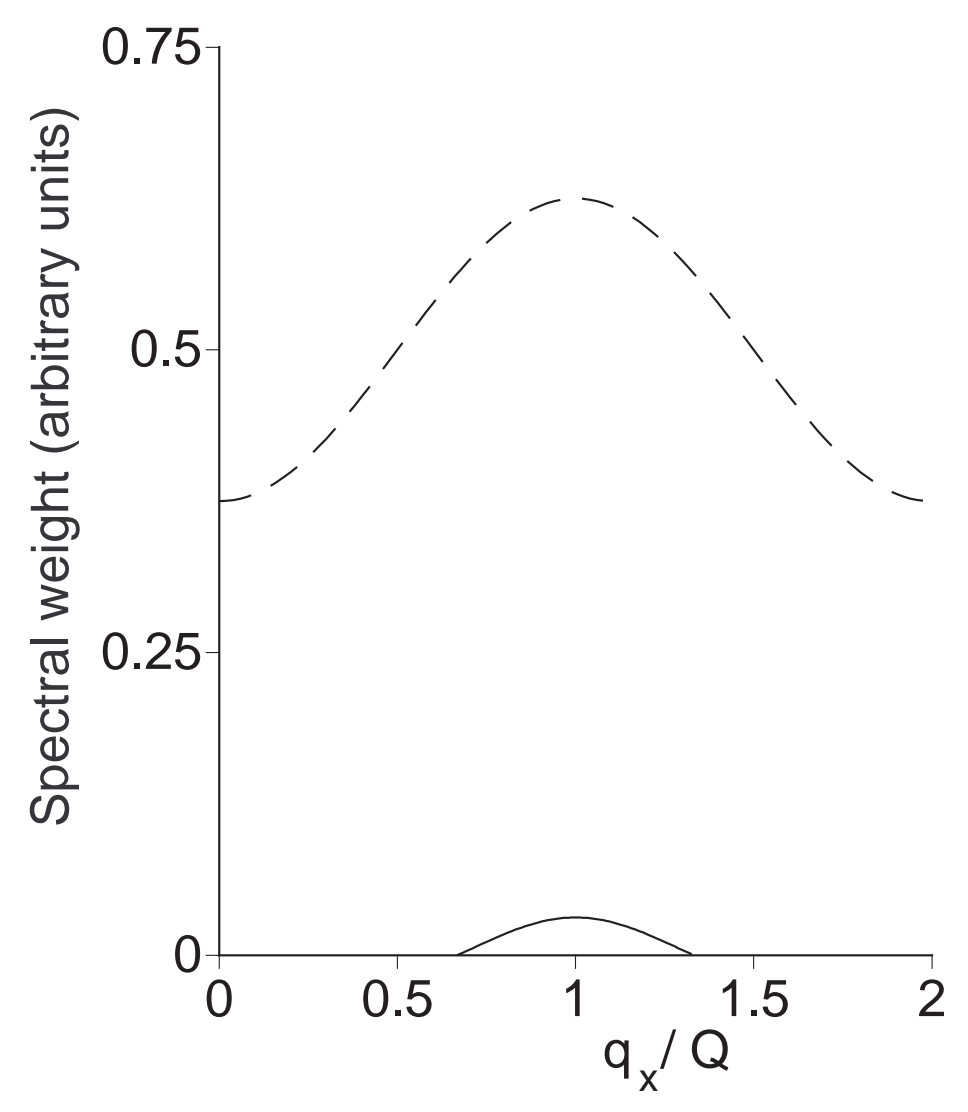

FIG. 4. Spectral weight in the single particle peak (dashed line) and the bound state peak (solid line) in $S(\vec{Q}, \omega)$ for a strongly coupled ladder (a typical value of $g=0.25$ is used). Note that the two curves actually correspond to different values of the transverve momentum $q_{y}$ chosen to maximize the respective spectral weights: the single particle part is shown for $q_{y}=\pi / d$ while the bound state part is shown for $q_{y}=0$ ( $d$ is the spacing along the rung of the ladder). 

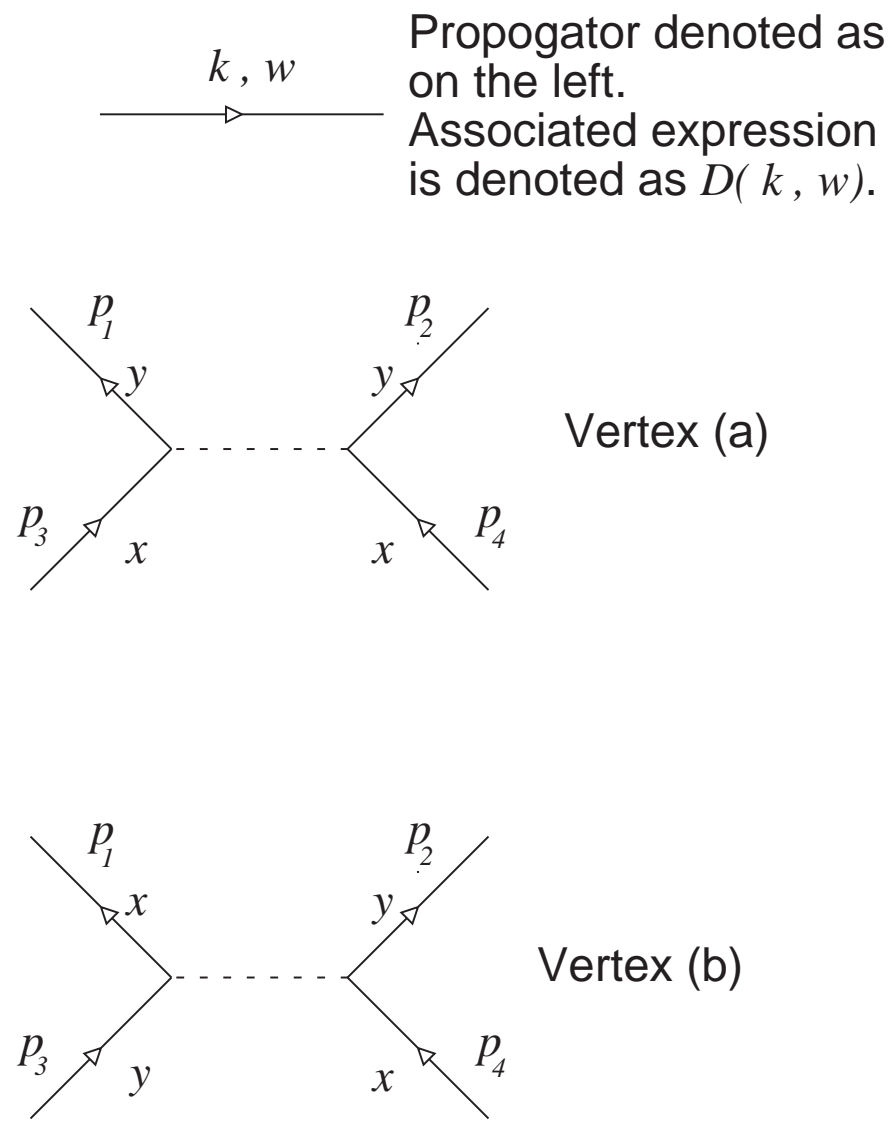

FIG. 5. The Feynman rules we need for the calculation described in Section II . The propogator $D(k, \omega)$ is given as $D(k, \omega)=i /(\omega-\varepsilon(k)+i \eta)$. The factor corresponding to the vertex (a) is $i g\left(f_{R}\left(p_{3}\right) \bar{f}_{R}\left(p_{1}\right) f_{L}\left(p_{4}\right) \bar{f}_{L}\left(p_{2}\right)+R \longleftrightarrow L\right) / 2$. The factor corresponding to (b) is $i g\left(f_{L}\left(p_{4}\right) \bar{f}_{R}\left(p_{1}\right)-f_{R}\left(p_{4}\right) \bar{f}_{L}\left(p_{1}\right)\right)\left(f_{L}\left(p_{3}\right) \bar{f}_{R}\left(p_{2}\right)-f_{R}\left(p_{3}\right) \bar{f}_{L}\left(p_{2}\right)\right)$. 

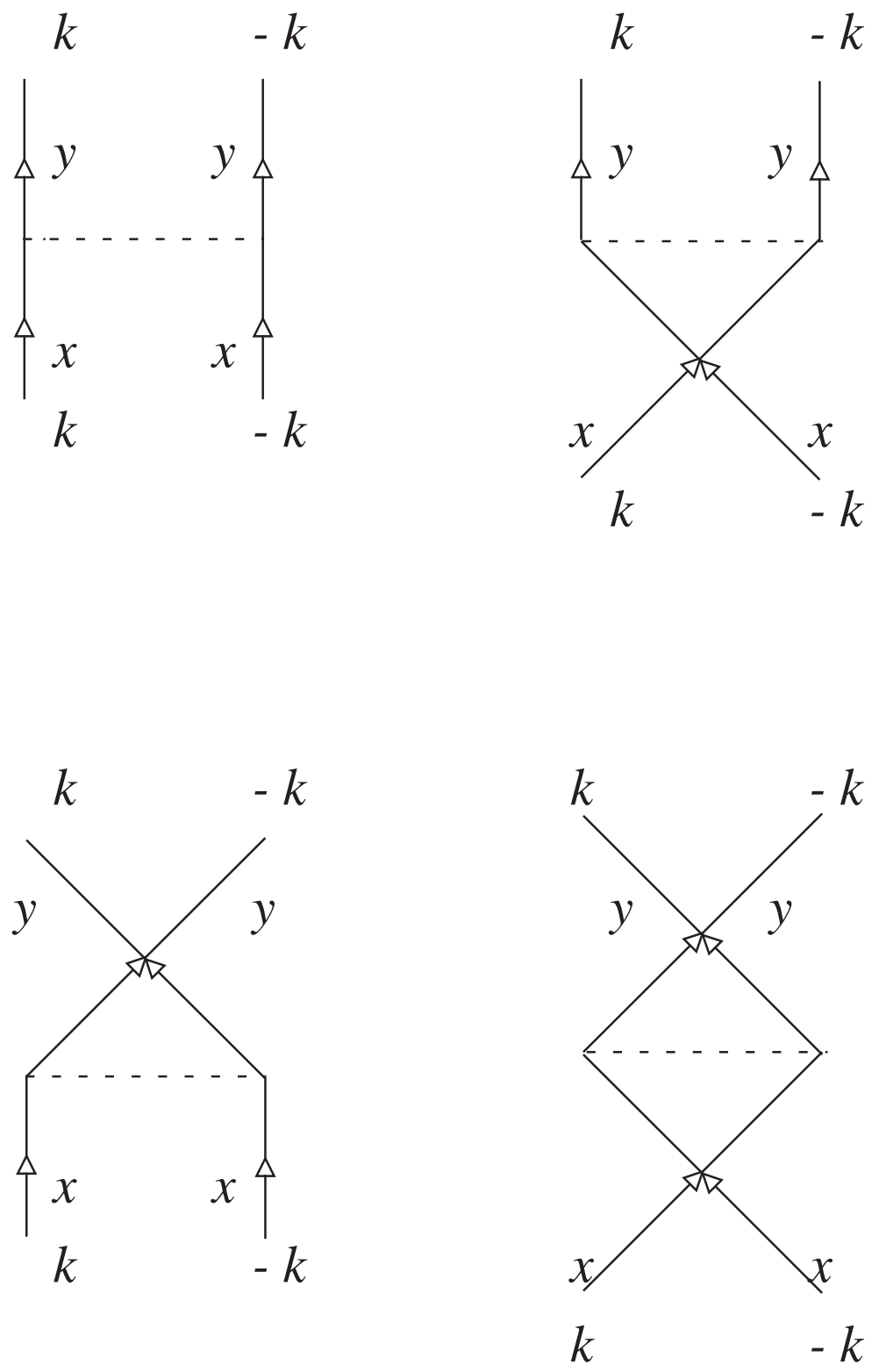

FIG. 6. Feynman diagrams contributing to $\Gamma_{4}(k x,-k x ; k y,-k y)$ to first order in $g$. All external lines carry on-shell frequencies corresponding to the momentum labels shown. 


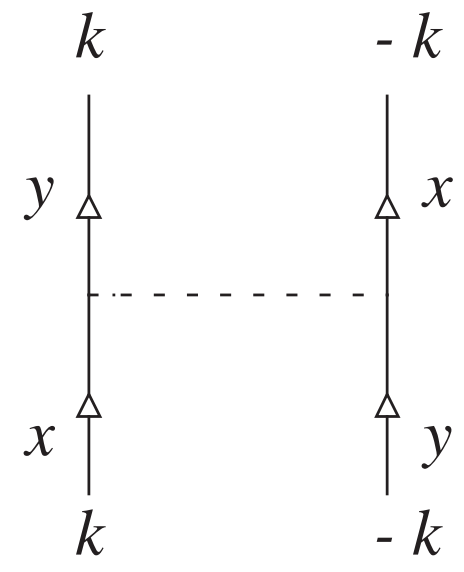

\section{Diagram (a)}

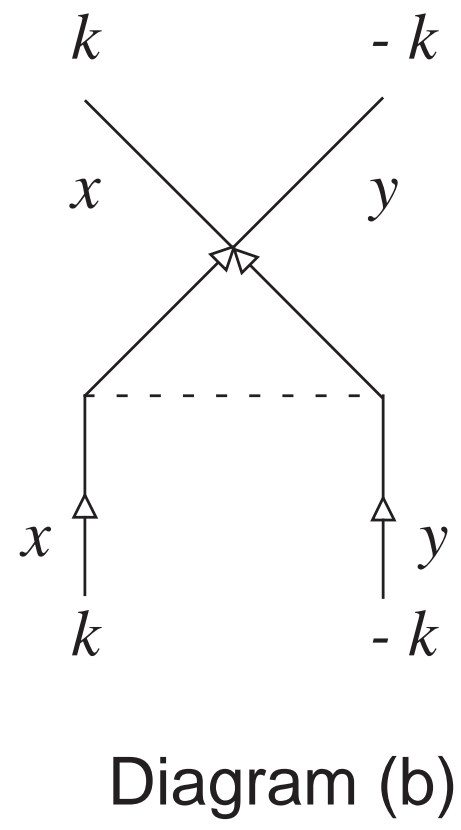

FIG. 7. Feynman diagrams contributing to $\Gamma_{4}(k x,-k y ; k y,-k x)$ (diagram (a)) and $\Gamma_{4}(k x,-k y ; k x,-k y)$ (diagram $\left.(\mathrm{b})\right)$ to first order in $g$. All external lines carry on-shell frequencies corresponding to the momentum labels shown. 

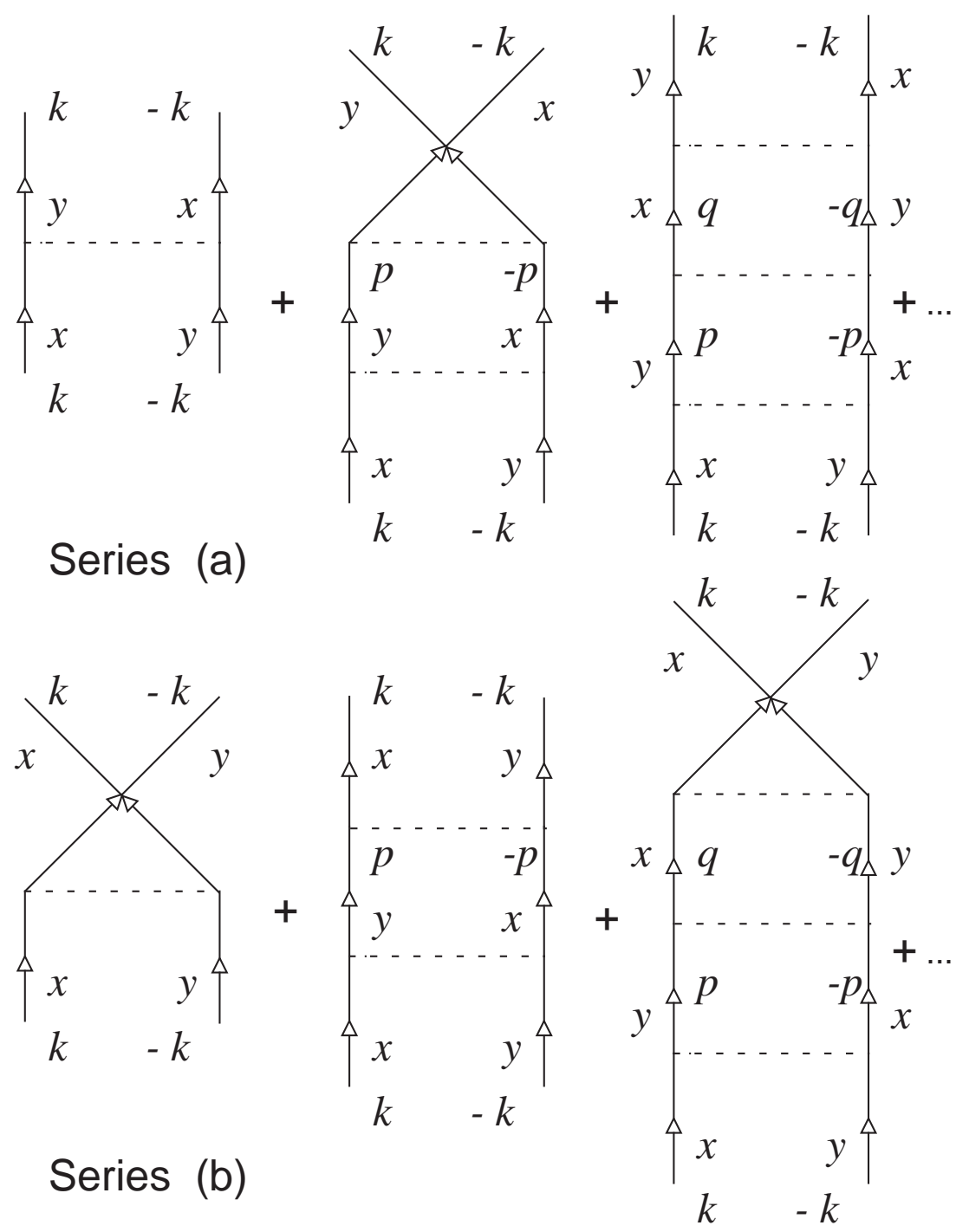

FIG. 8. Ladder series giving the leading infrared divergent terms in the expansion for $i M_{3}$ (diagram (a)) and $i M_{2}$ (diagram (b)). All external lines carry on-shell frequencies corresponding to the momentum labels shown. The internal lines also carry frequency labels that are not explicitly shown. 


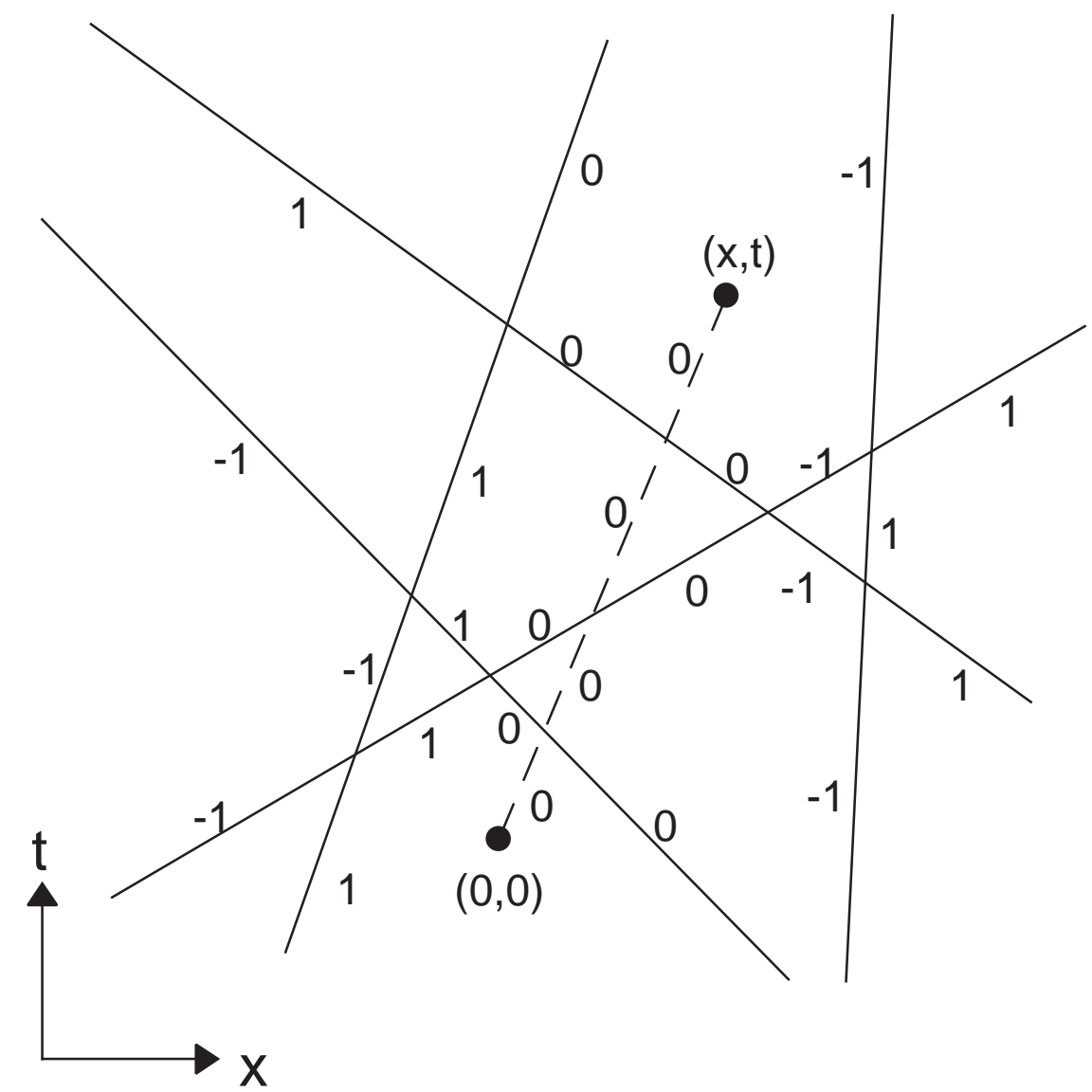

FIG. 9. A typical set of particle trajectories contributing to $C(x, t)$. Each full line represents paths traversed by particles moving both forward and backward in time. The dashed line is traversed only going forward in time. Shown on the trajectories are the values of the particle spins $m_{k}$ which are independent of $t$ in the low $T$ limit. Notice that all the trajectories intersecting the dashed line have a spin equal to that of the dashed line: only such configurations contribute to the $C(x, t)$. 


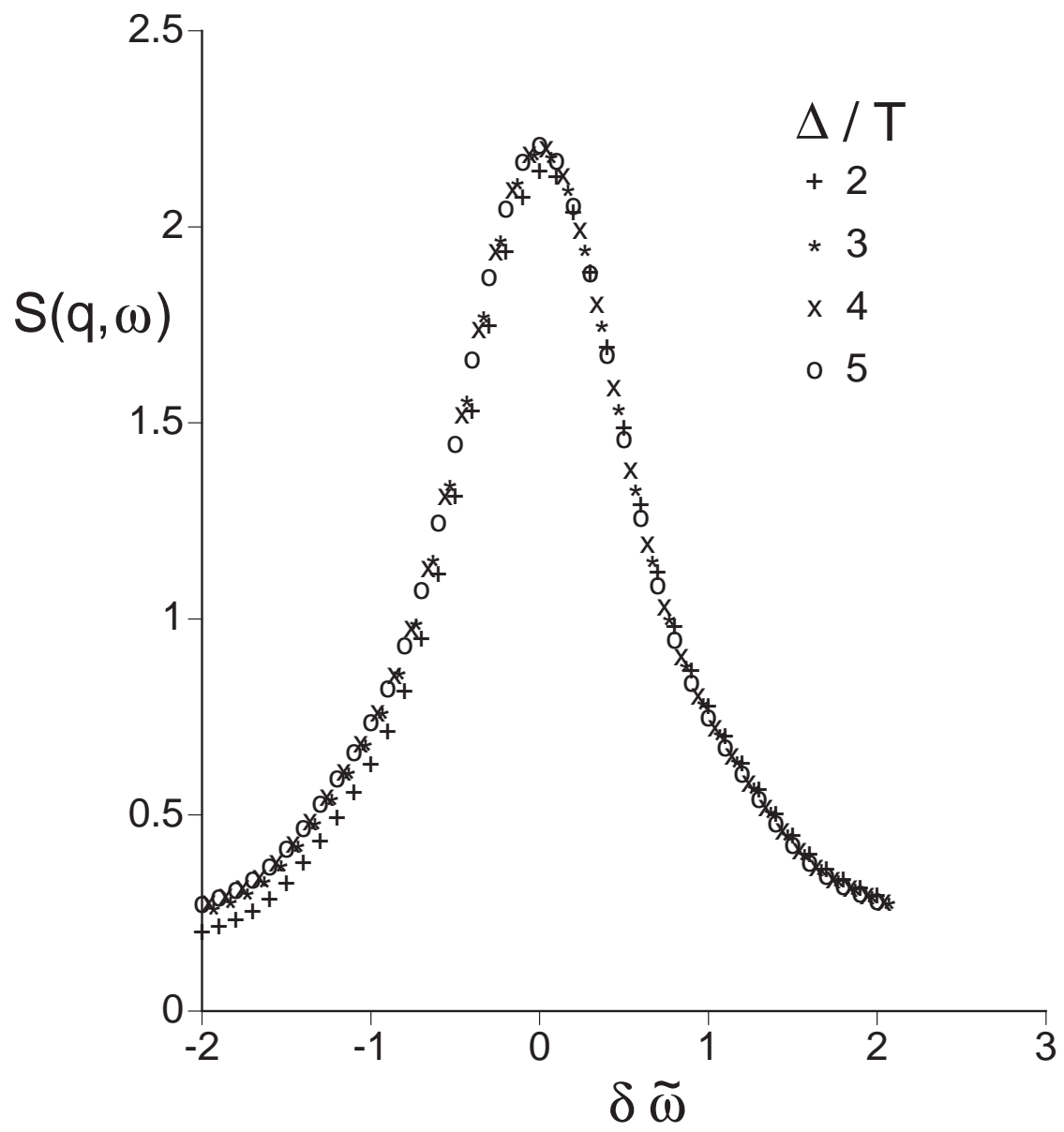

FIG. 10. $S(q, \omega)$ rescaled by a factor of $\mathcal{A} c L_{t} /\left(\pi^{2} \Delta\right)$ plotted against $\delta \tilde{\omega}=L_{t}(\omega-\Delta)$ with $q=\pi / a$ for $\Delta / T=2,3,4$, and 5 . Note the scaling collapse of the curves corresponding to the three lowest temperatures. 


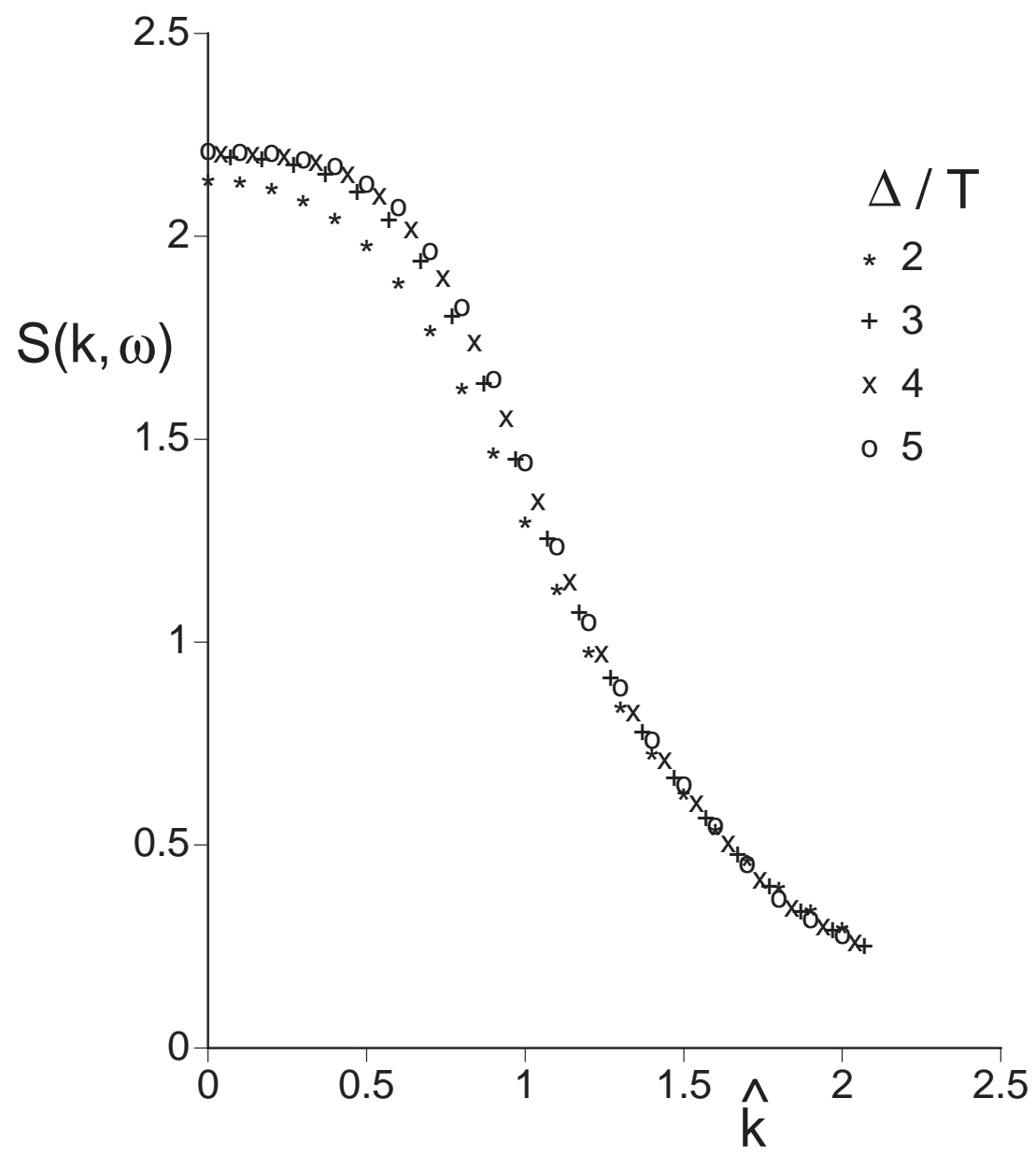

FIG. 11. $S(\pi / a+k, \omega)$ rescaled by a factor of $\mathcal{A} c L_{t} /\left(\pi^{2} \Delta\right)$ plotted against the rescaled variable $\hat{k}=k c\left(L_{t} / \Delta\right)^{1 / 2}$ with $\omega=\Delta$ for $\Delta / T=2,3,4$, and 5 . Again, note the scaling collapse of the curves corresponding to the three lowest temperatures. 


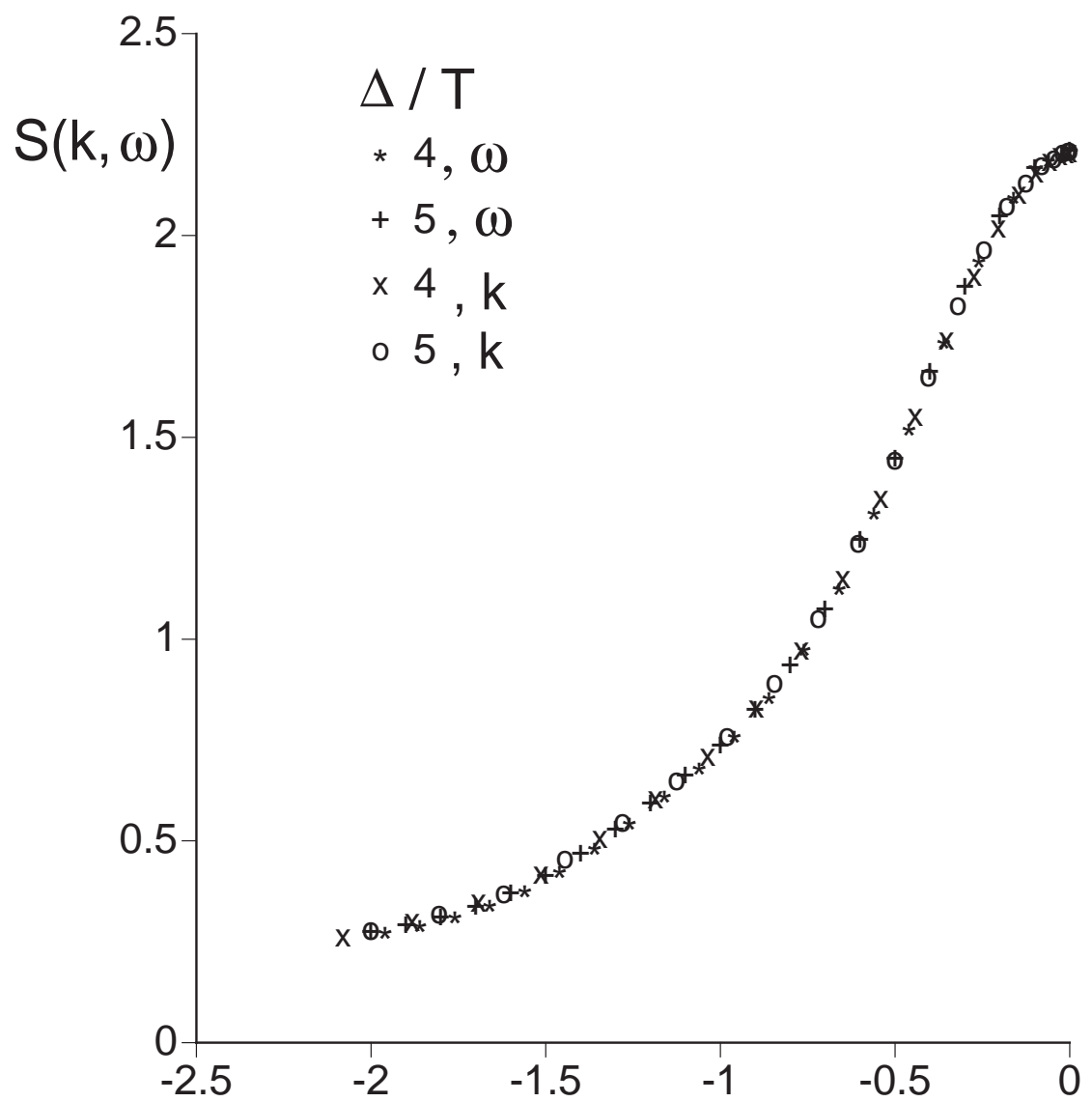

FIG. 12. The scaling curve of Fig 11 (defined by the data for $\Delta / T=4$ and $\Delta / T=5$ ) plotted against the independent variable $\left(-\hat{k}^{2} / 2\right)$ compared to the scaling curve of Fig 10 (again defined by the data for $\Delta / T=4$ and $\Delta / T=5$ ) for the corresponding negative values of $\delta \tilde{\omega}$. The two coincide within our numerical errors. 


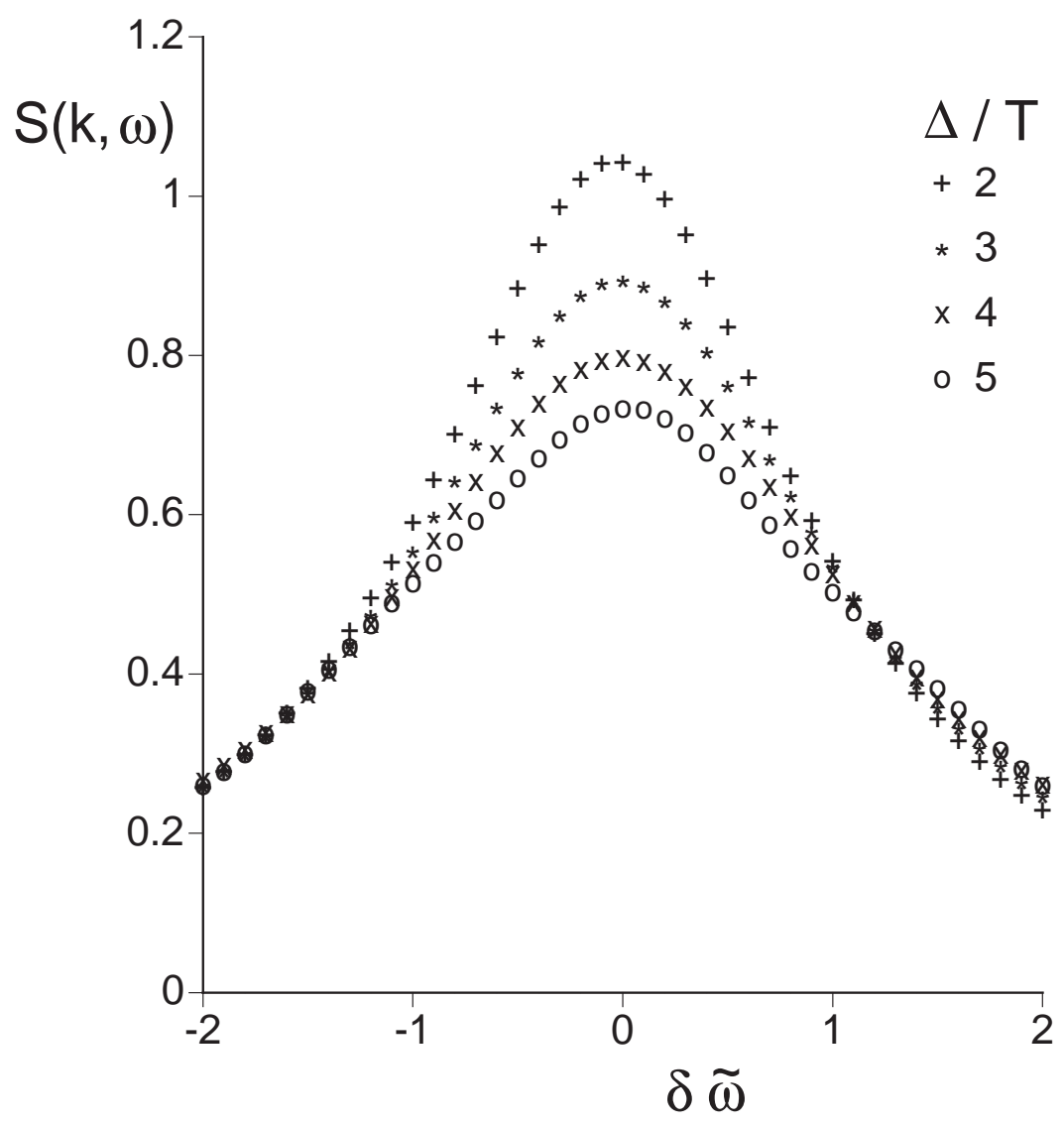

FIG. 13. $S(k+\pi / a, \omega)$ rescaled by a factor of $\mathcal{A} c L_{t} /\left(\pi^{2} \Delta\right)$ plotted against $\delta \tilde{\omega}=L_{t}\left(\omega-2^{1 / 2} \Delta\right)$ with $k=\Delta / c$ for $\Delta / T=2,3,4$, and 5 . 


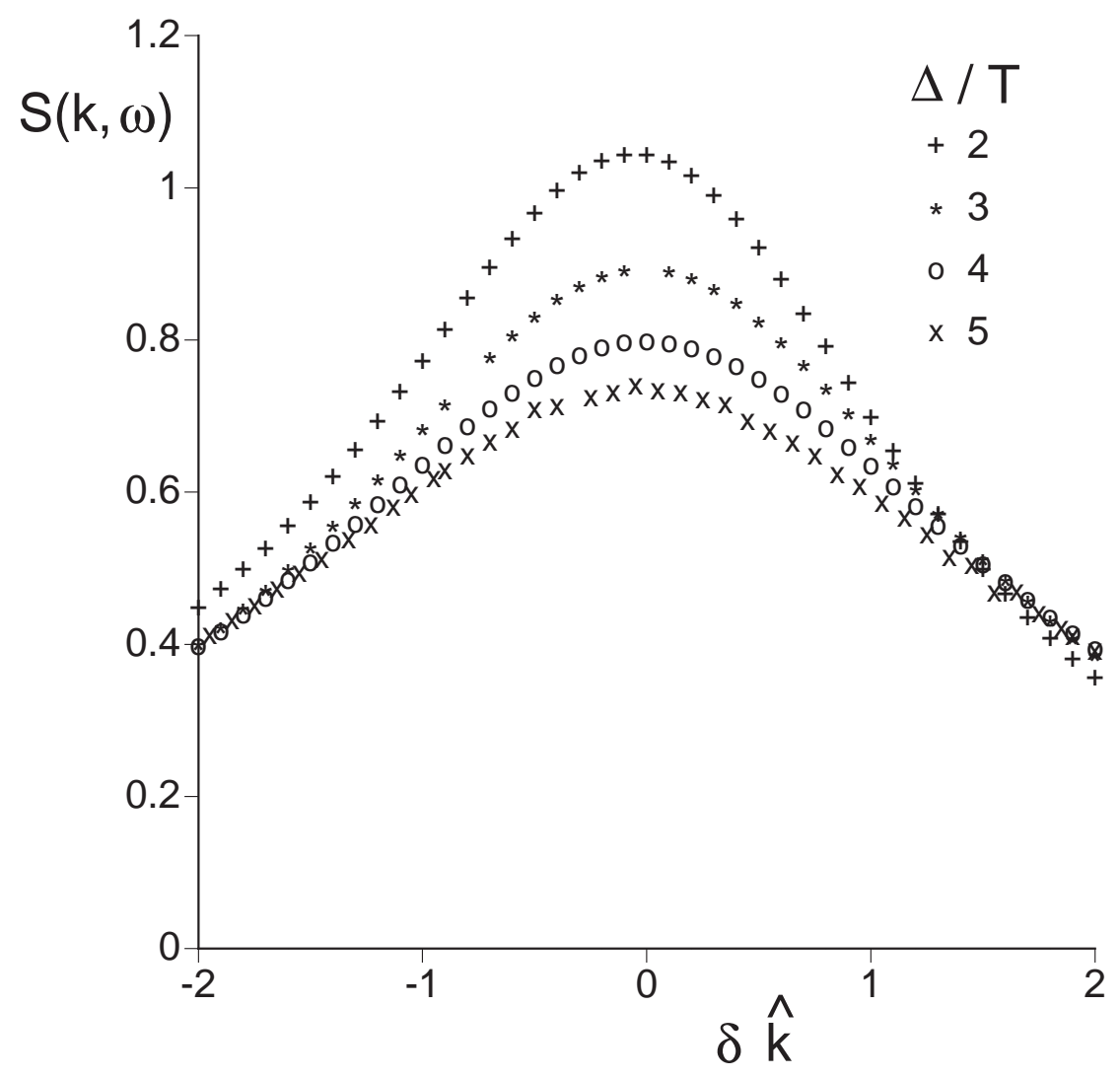

FIG. 14. $S(k+\pi / a, \omega)$ rescaled by a factor of $\mathcal{A} c L_{t} /\left(\pi^{2} \Delta\right)$ plotted against the rescaled variable $\delta \hat{k}=c L_{t}(k-\Delta / c)$ with $\omega=2^{1 / 2} \Delta$ for $\Delta / T=2,3,4$, and 5 . 


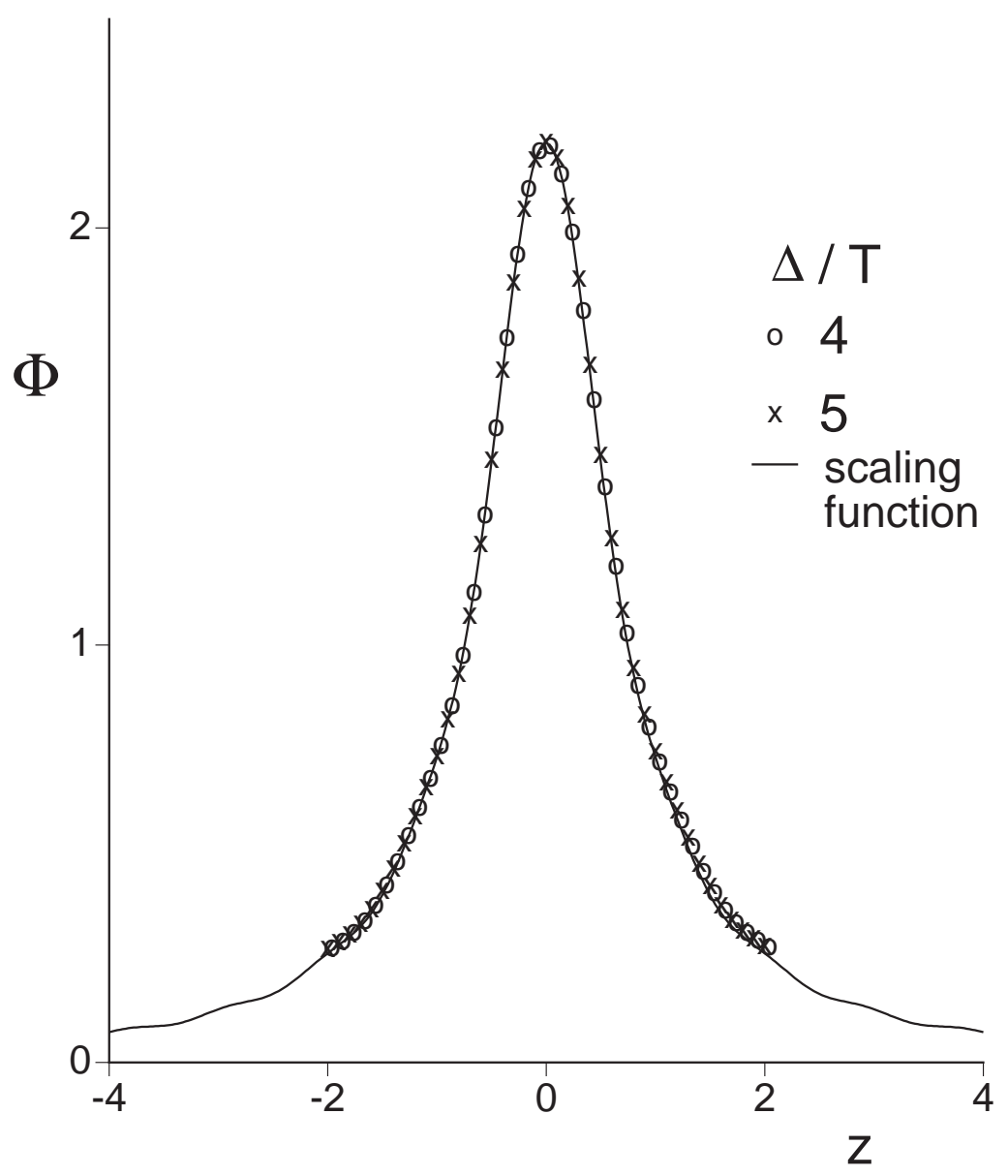

FIG. 15. The scaling function $\Phi(z)$ determined directly from Eqn 3.12 compared with the scaling curve defined by the results already shown in Fig 10 and Fig 12 


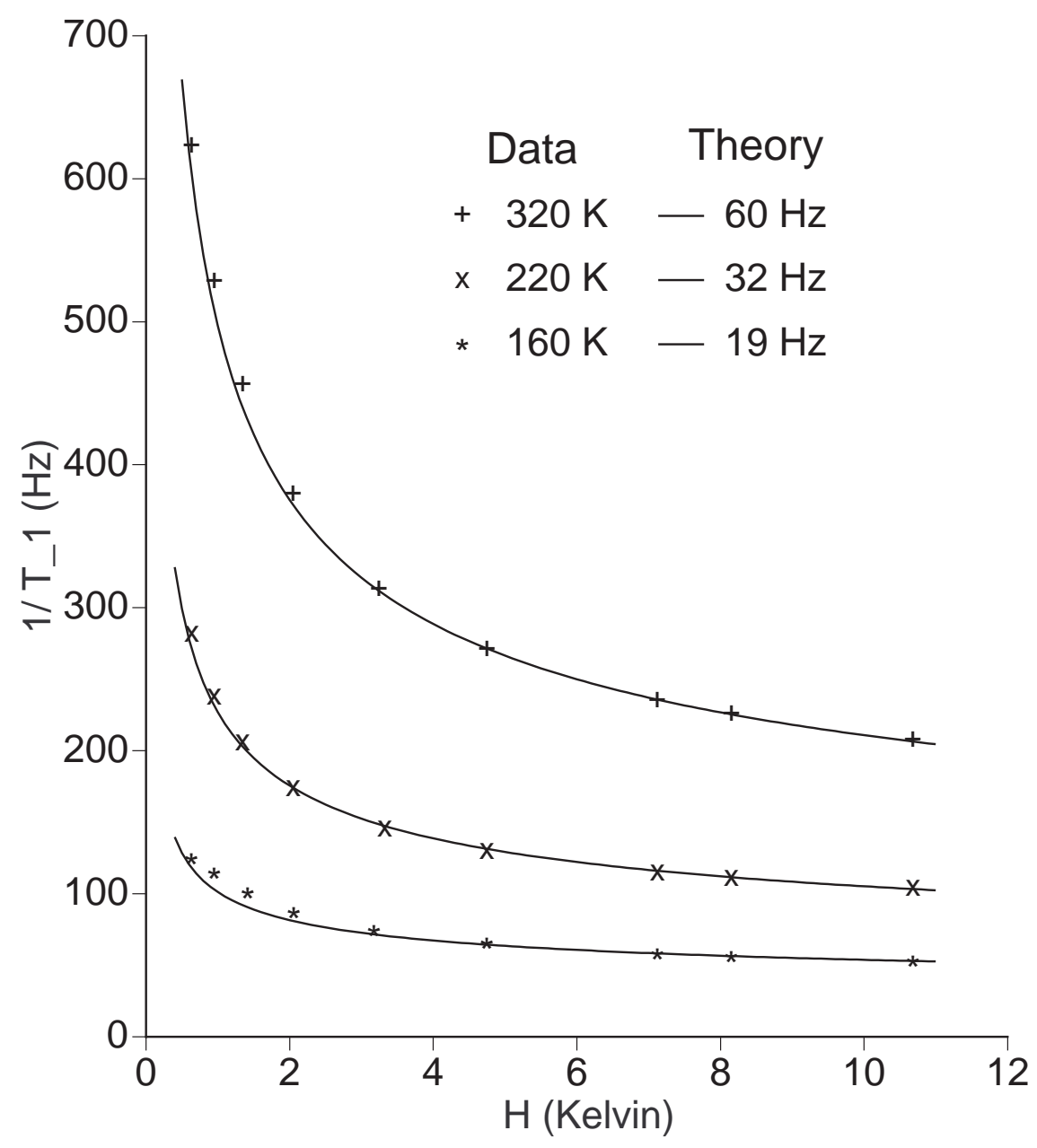

FIG. 16. Field dependence of $1 / T_{1}$ for $T>120 \mathrm{~K}$. The experimental data of [30] is compared with the theoretical predictions offset by a field-independent background rate $R_{b}$ which is the only free parameter of the fit; the fit value of $R_{b}$ is shown under the theory column. 


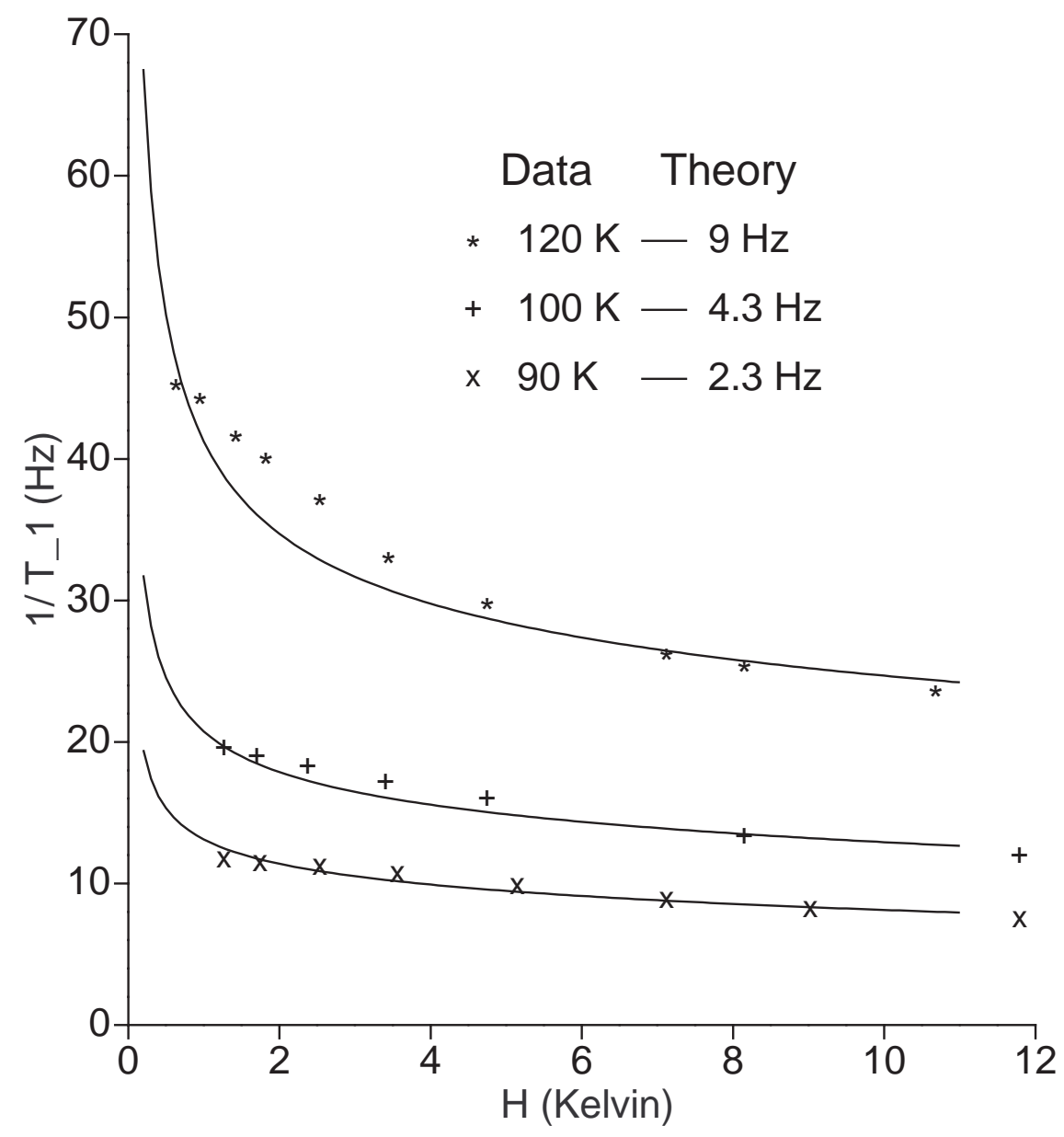

FIG. 17. Field dependence of $1 / T_{1}$ for a few temperatures $T<120 \mathrm{~K}$. The experimental data of [30] is compared with the theoretical predictions offset by a field-independent background rate $R_{b}$ which is the only free parameter of the fit; the fit value of $R_{b}$ is shown under the theory column. 


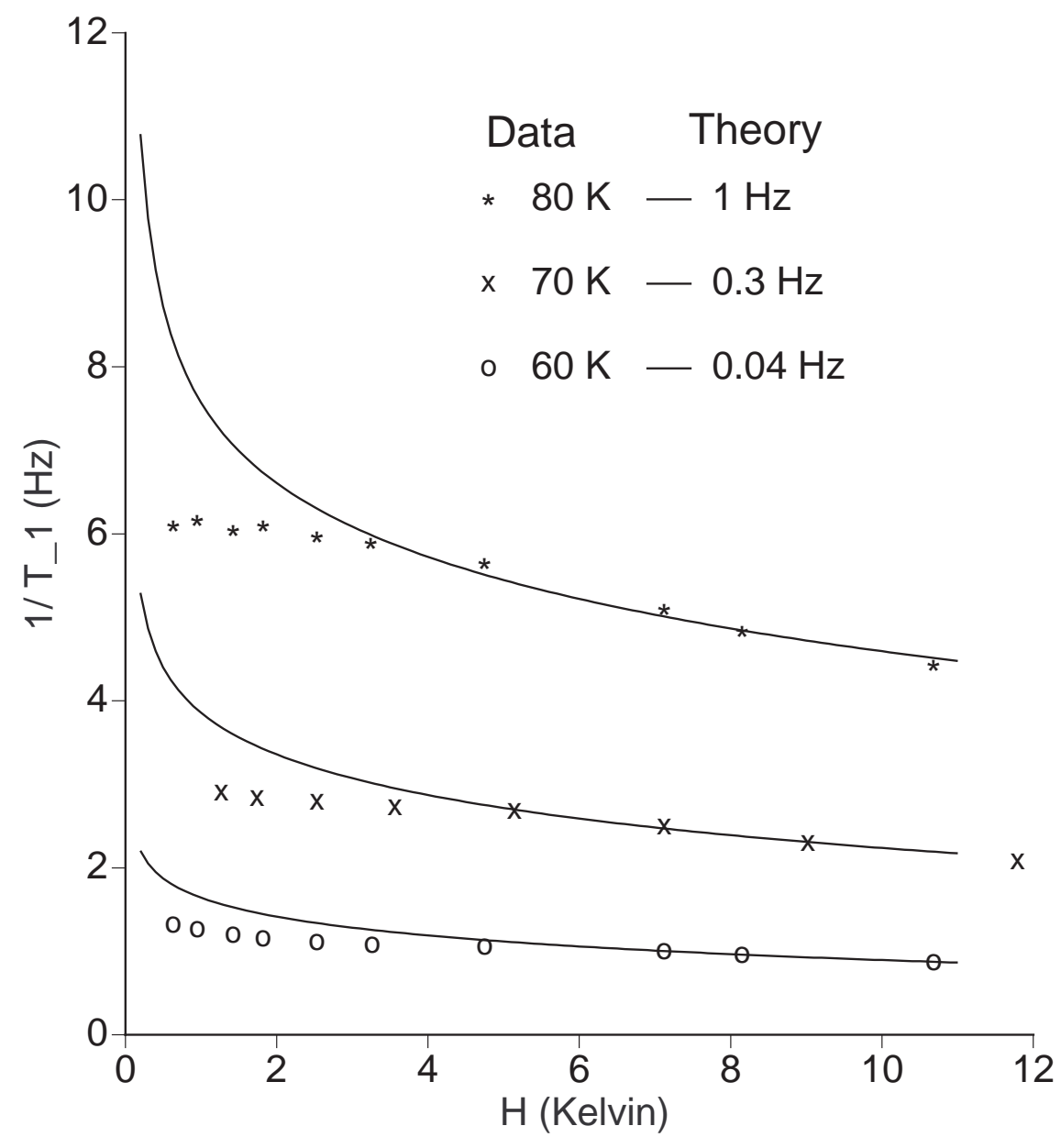

FIG. 18. Field dependence of $1 / T_{1}$ for the lowest temperatures for which data is available. The experimental data of [30] is compared with the theoretical predictions offset by a field-independent background rate $R_{b}$ which is the only free parameter of the fit; the fit value of $R_{b}$ is shown under the theory column. 


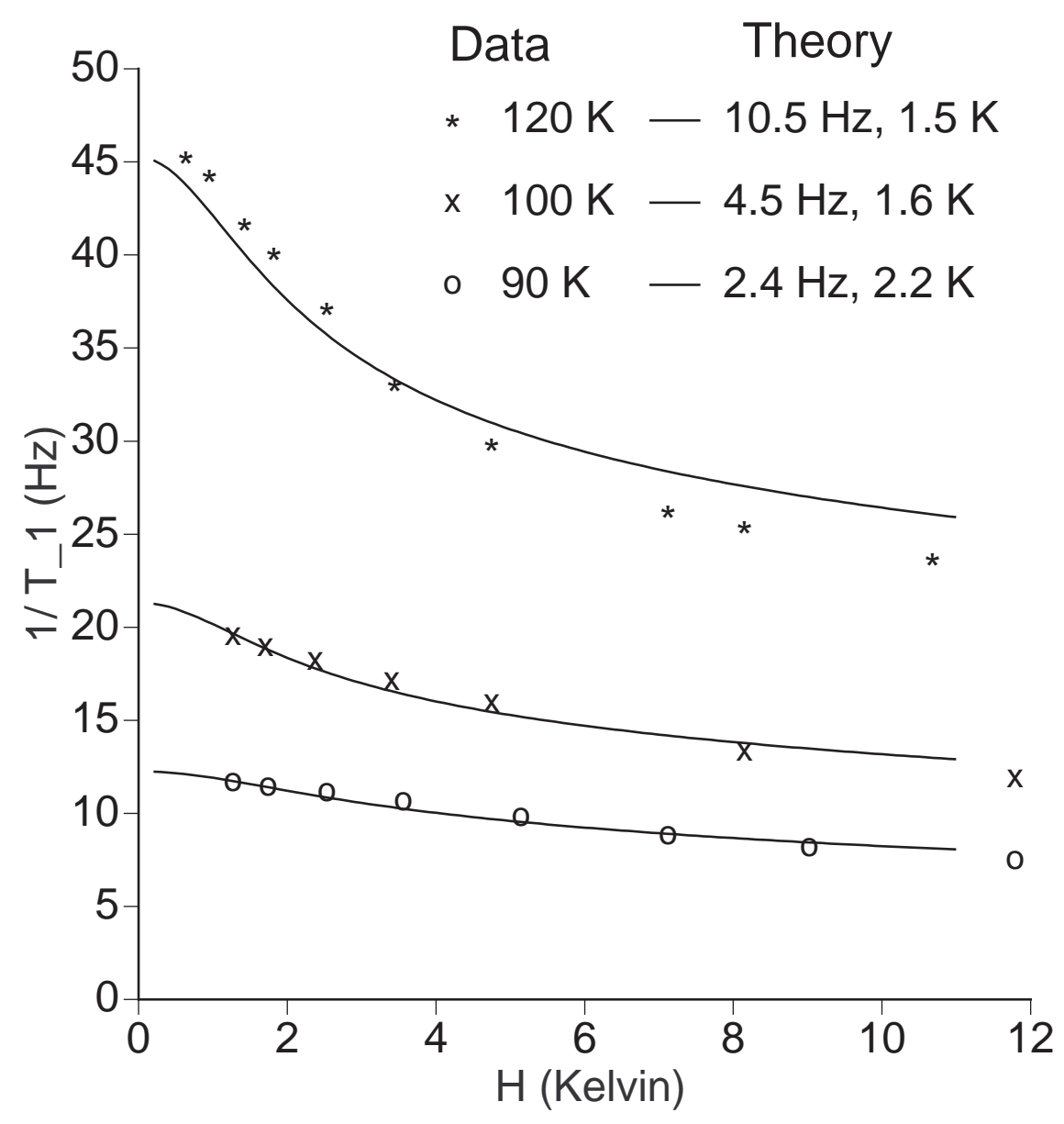

FIG. 19. Field dependence of $1 / T_{1}$ fit to the phenomenological form described in the text. The experimental data of [30] at $T=120,100$, and $90 \mathrm{~K}$ is compared to our phenomenological form that incorporates a spin dissipation rate $\gamma$ in addition to a field independent background rate $R_{b}$. The values of $R_{b}, \gamma$ are listed under the theory column. 


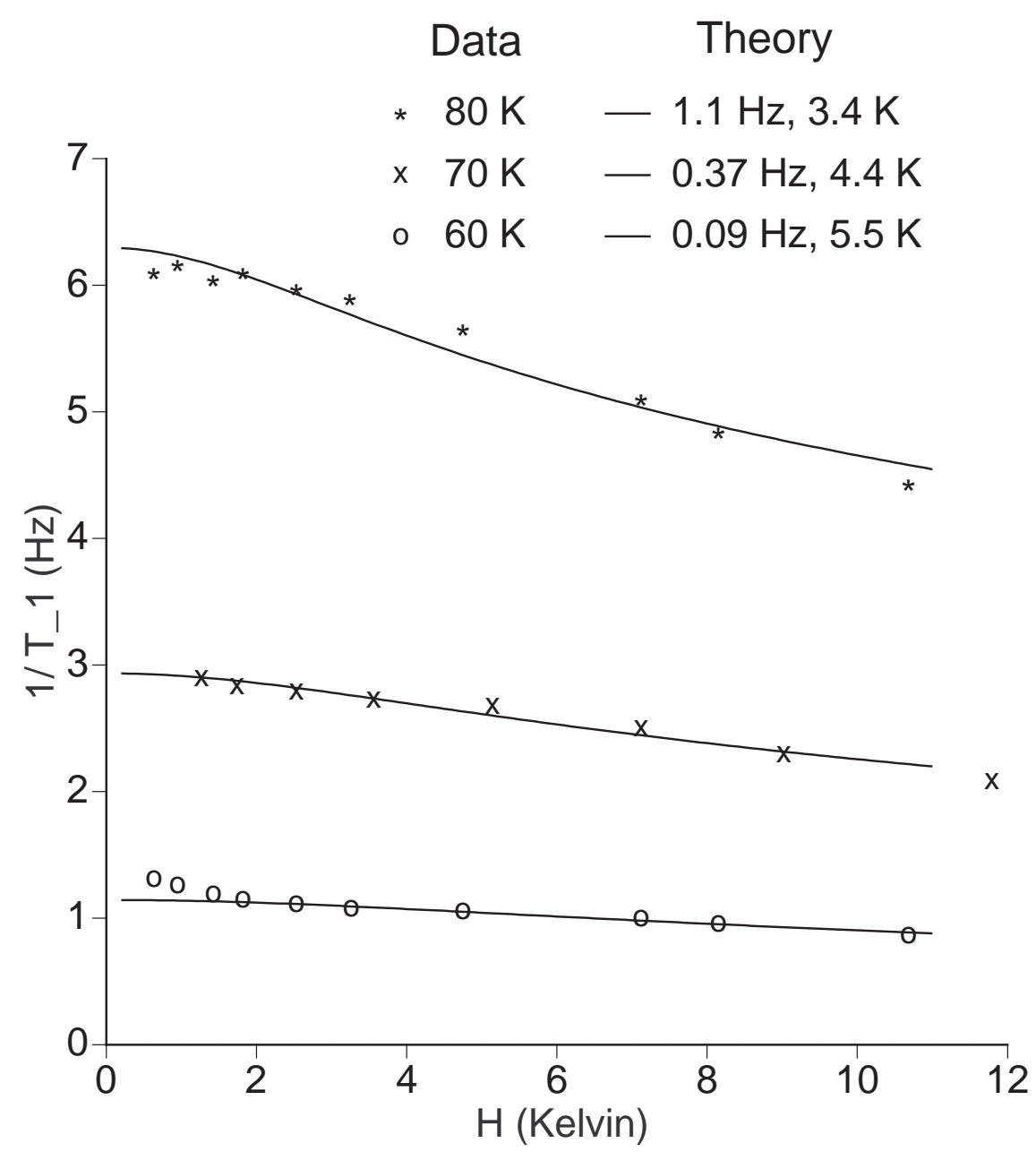

FIG. 20. Field dependence of $1 / T_{1}$ fit to the phenomenological form described in the text. The experimental data of [30] at $T=80,70$, and $60 \mathrm{~K}$ is compared to our phenomenological form that incorporates a spin dissipation rate $\gamma$ in addition to a field independent background rate $R_{b}$. The values of $R_{b}, \gamma$ are listed under the theory column. 


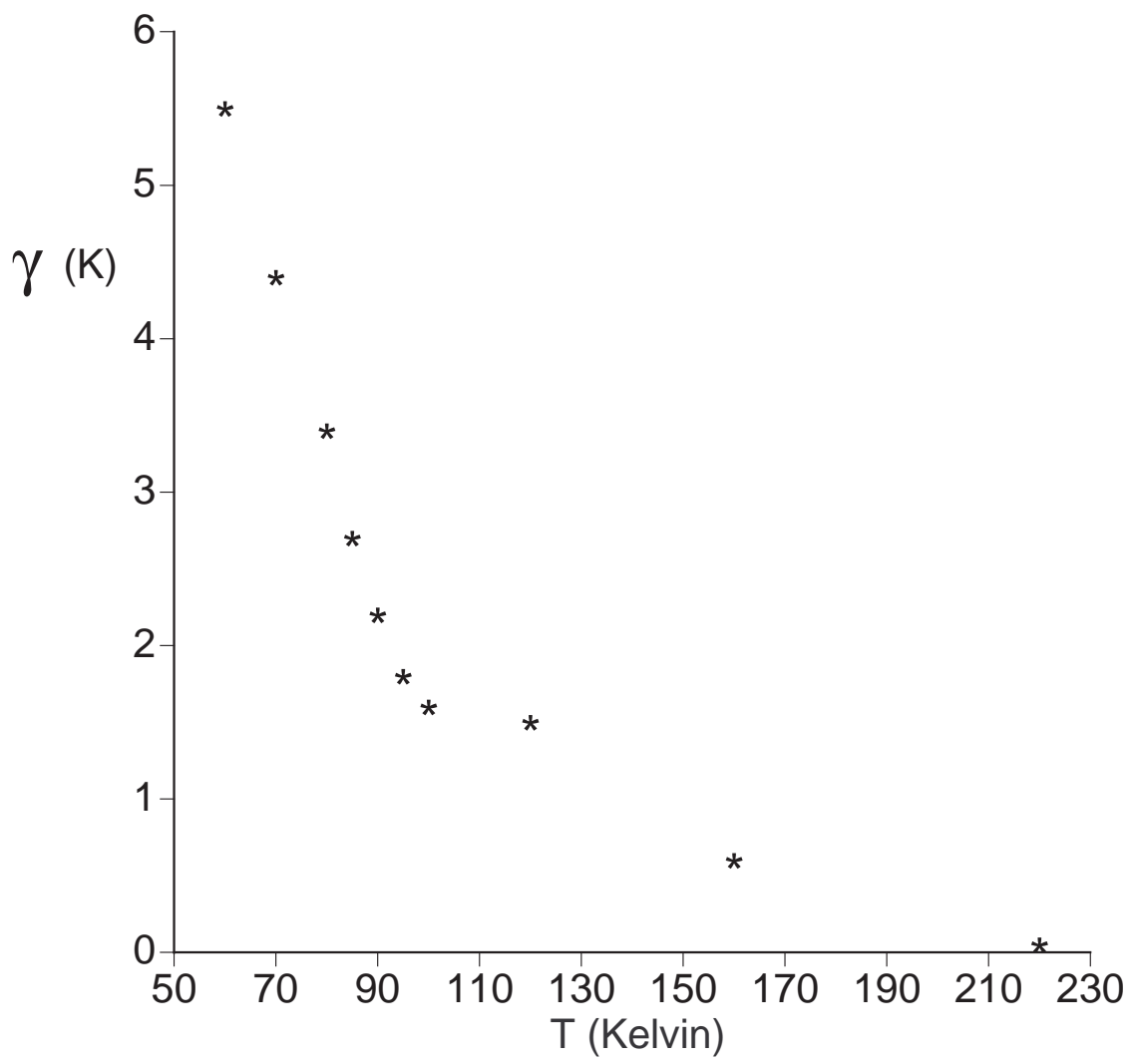

FIG. 21. Temperature dependence of the spin-dissipation rate $\gamma$ determined by fitting our phenomenological form for $1 / T_{1}$ to the experimental data of [30]. 


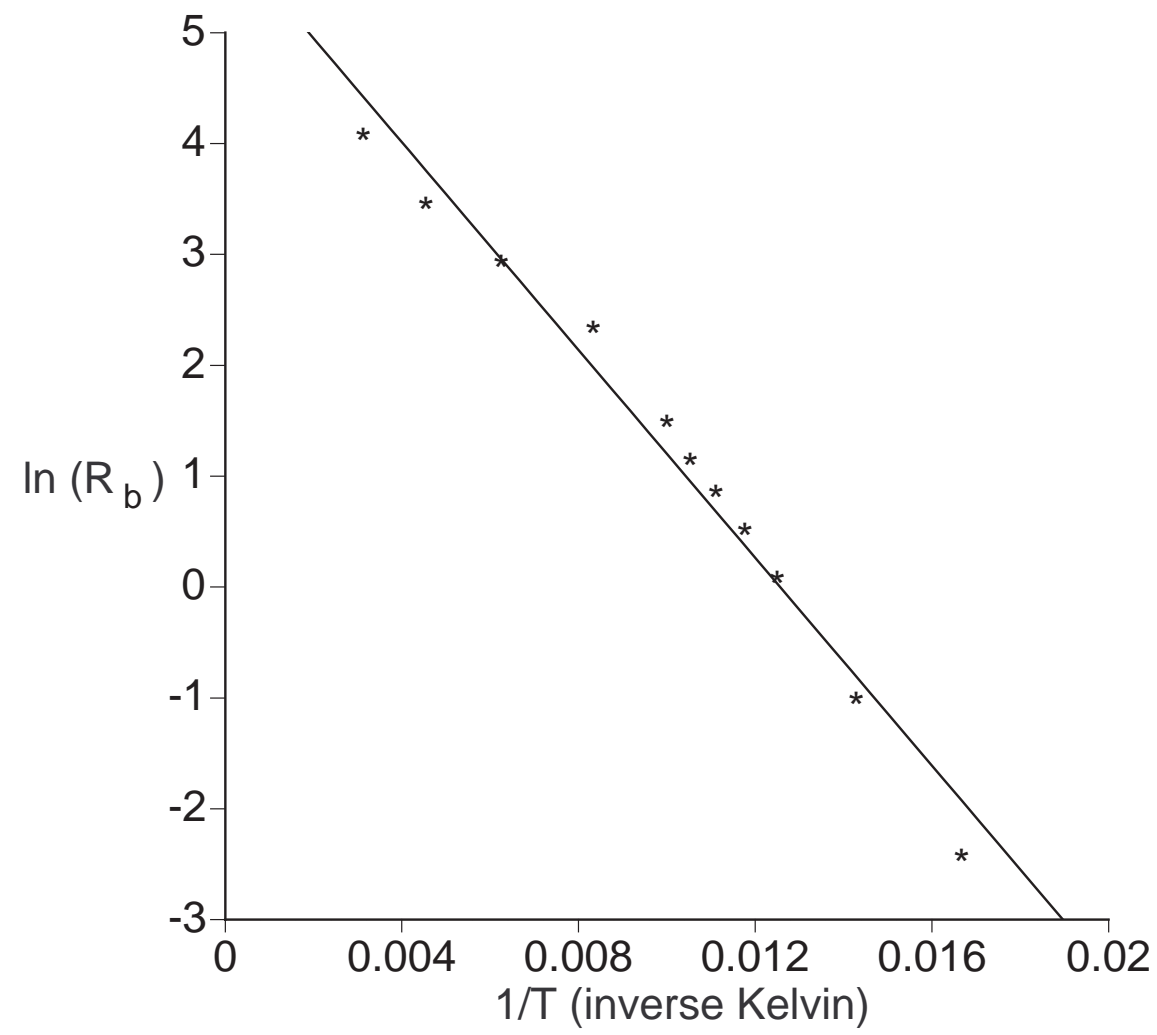

FIG. 22. Temperature dependence of the background rate $R_{b}$ determined by fitting our phenomenological form for $1 / T_{1}$ to the experimental data of [30]. We plot $\ln \left(R_{b}\right)$ against $1 / T$ to check for activated behaviour and indeed find an approximate linear relation, the best fit for the slope being $468 \mathrm{~K}$. 


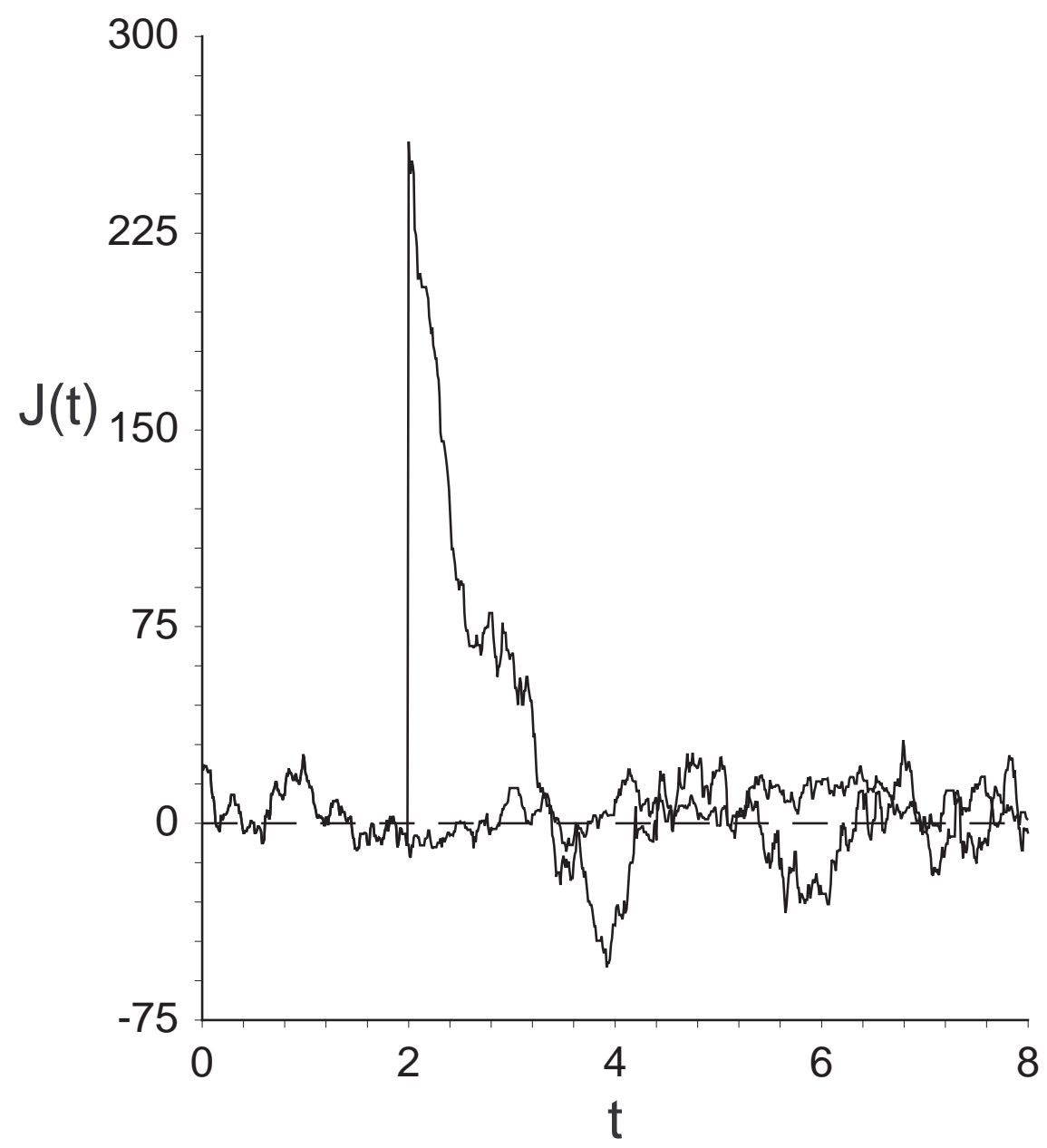

FIG. 23. Deterministic time evolution of the spin current $J(t)$ (defined in (A6 ) ) for two systems of 400 particles on a circle with the same initial condiions; the value of $J(t)$ changes in discrete steps at each collision between a pair of particles. For one of systems, there is an impulse in velocities given by (A8) at a time $t_{0}=2$. This produces a macroscopically significant $J(t)$, which however decays away in a few collision times. The only remnant of the impulse is a 'heating' of the system, reflected in the larger amplitude of the order $\sqrt{N}$ fluctuations in $J(t)$ for the impacted system. 